\title{
SPATIOTEMPORAL NONLINEAR OPTICS IN MULTIMODE FIBERS
}

\author{
A Dissertation \\ Presented to the Faculty of the Graduate School \\ of Cornell University \\ in Partial Fulfillment of the Requirements for the Degree of \\ Doctor of Philosophy
}

\author{
by \\ Logan Gary Wright
}

May 2018 
(C) 2018 Logan Gary Wright

ALL RIGHTS RESERVED 


\section{SPATIOTEMPORAL NONLINEAR OPTICS IN MULTIMODE FIBERS Logan Gary Wright, Ph.D.}

Cornell University 2018

Multimode nonlinear fiber optics is a microcosm, a hyperspace of possibilities embedded within a niche of a niche of science. It is full of phenomena that transcend dimensionality (these provocative statements will be clarified). It could inform new generations of compact, cost-efficient scientific tools, or major upgrades to optical fiber telecommunications. These advances in foundational, infrastructural technologies would have major impact on the human world, from science and knowledge through to the economic engine of the internet.

In this thesis, I examine the work of my PhD that has explored nonlinear pulse propagation in multimode fibers. Both as a microcosm of science, a mostly-mundane story of half-reasoned exploration, and as a rich and complex world of observed phenomena and inspired applications, here I attempt to retrospectively describe the work and science in as coherent a summary form as can be managed at this point. We examine first multimode solitons in anomalous dispersion fibers. These are stable configurations of light in nonlinear multimode fiber and, in some sense, the conceptual building blocks or framework through which we can understand much more complex dynamics of multimode supercontinuum generation. We then examine the complex mode coupling in nonlinear, disordered multimode fibers, and attempt to integrate the multitude of effects into a cohesive framework of modal energy flow. Finally, we explore multimode fiber lasers and spatiotemporal mode-locking, the most general form of coherent light self-organization in an optical oscillator. 


\section{BIOGRAPHICAL SKETCH}

Logan G. Wright grew up in Weyburn, Saskatchewan, Canada. He is from there. From 2008 to 2012, he studied Engineering Physics at Queen's University, in Kingston, Ontario, Canada, for reasons which in retrospect are not precisely known. He then came to Cornell University, in the United States, for a PhD. in Applied Physics in 2012. He joined Frank Wise's group in 2013, again for reasons which in retrospect are not precisely known. After receiving his $\mathrm{PhD}$, it seems likely he will do something for reasons which will be, in retrospect, not precisely knowable but also will, again in retrospect, seem to be precisely the correct decision. This is what we call in science 'serendipity' or 'emergence', which are to put conceptual caps on the notion that things have a miraculous, if not always predictably, way of working themselves out. 
This document is dedicated to my family. 


\section{ACKNOWLEDGEMENTS}

In this format, it's genuinely hard to convey the thanks I ought to with the sincerity and fullness that it deserves. Still, here is the attempt.

I owe much to the teachers I had as a child who cared enough to treat me as an individual. This includes many, but Lars Guenther and Karen Kennedy-Allin are two whose names stand out.

At Queen's, I had a similar privilege of devoted, talented educators. Geoff Lockwood, Brad Diak, Robert Gooding, Marc Dignam and Stephen Hughes seem especially important and inspiring in retrospect. I am also thankful to have been in such a brilliant and supportive Engineering Physics class at Queen's.

Nobody could design a better first science advisor than Simon Hesp. James Fraser has been and remains an important role model, as a person, teacher, group leader and researcher. I owe much inspiration and gratitude to James' student Paul Webster, who patiently and enthusiastically mentored me through this time. Thanks also to Kevin Resch, and in particular his student Jon Lavoie who, with incredible patience and rigor, trained me to do experimental optics.

At Cornell, I have had the privilege of working with a long list of colleagues and friends, each of whom has inspired me and taught me something (most often many somethings). Will Renninger and Hui Liu thoughtfully trained me and my colleagues. I try hard to emulate the attitudes to research and life that Will and Hui each have. Will in particular has remained an important, valued colleague after leaving Cornell. Erin Lamb exemplifies 'applied physicist', and I appreciate her leadership and example in the broadest sense. Yuxing Tang is

a model of hard-working, positive thinking, and human interaction. Zhanwei Liu, meanwhile, has been an important friend and colleague throughout this research, and the source of a huge amount of learning. I likewise appreciate 
Walter Fu, Zimu Zhu, and Pavel Sidorenko, who with their competency make it easier for me to leave Frank's group, but otherwise make it bittersweet.

I have been lucky to advise several students in some capacity: Lili Huang, Zach Ziegler and Andrei Isichenko, as well as Yi-Hao Chen. It is hard for me to imagine this mentorship has taught them more than I have learned. I also thank Yi-Hao and his contemporary Michael Buttolph in advance for their success as part of Frank's group.

Frank Wise is a dedicated scientist who sees his students' success and development as his top priority, and who mentors with humility and patience well-worth emulation. I also hope to emulate Frank's open-minded eagerness to learn from his colleagues (including his students, even) - he is always improving as a scientist and group leader. Last, for his ethics and responsibility as a member of the wider scientific community. Frank does things the right way.

Demetri Christodoulides has been an invaluable complement to Frank, as have other collaborators, notably Dan Nolan, Joel Carpenter and Boris Malomed. Thanks also to my committee members: Clifford Pollock, whose student Victor I owe numerous lessons to, and Alex Gaeta, who inspired my decision to come to Cornell. More broadly, I have been lucky to learn from many other colleagues from all over the world, who have without exception significantly enhanced my science as well as my scientific education.

For my family the language that until here has flown quite freely begins to break down and become too clearly insufficient. But having tried everything from music to mathematics to scientific writing itself... I thank my parents for their unbreakable support, their strong values and empathy, and for the examples they set. Likewise, I appreciate the support of others in family. And Blaine. My love, partner, and best friend, thank you. 


\section{TABLE OF CONTENTS}

Biographical Sketch . . . . . . . . . . . . . . . . iii

Dedication ..................... iv

Acknowledgements . . . . . . . . . . . . . . . . v

Table of Contents . . . . . . . . . . . . . . . . . . . vii

List of Tables . . . . . . . . . . . . . . . . . ix

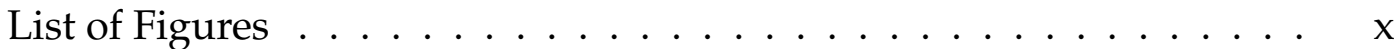

0.1 Foreword: a guide to this thesis $\ldots \ldots \ldots \ldots \ldots \ldots$

1 Introduction 6

1.1 Motivations .................... 6

1.2 The theoretical basics of multimode nonlinear fiber optics . . . . . 10

1.2.1 The 3+1 D generalized nonlinear Schrödinger equation . . 10

1.2 .2 Modes . . . . . . . . . . . . . . . . . . . . 12

1.2.3 The multimode nonlinear Schrödinger equations . . . . . 16

1.2.4 The nonlinear term(s) . . . . . . . . . . . . . . 17

1.3 More specific motivations . . . . . . . . . . . . . . . . . . 19

1.3.1 Peak power of ultrafast fiber lasers . . . . . . . . . . 20

1.3.2 Information capacity in optical fiber telecommunications . 21

1.3.3 Exploring the world of multimode nonlinear optical pulse propagation . . . . . . . . . . . . 26 26

1.4 Experiments in multimode nonlinear fiber optics . . . . . . . . . 28

1.4.1 The 'mode-us' operandi of MMNLFO . . . . . . . . . . . . 29

2 Spatiotemporal dynamics of multimode optical solitons 32

2.1 Linear propagation of pulses in multimode fibers . . . . . . . . 34

2.2 Multimode solitons . . . . . . . . . . . . . . . . 36

2.2.1 Solitons in $1+1 \mathrm{D} \ldots \ldots \ldots \ldots$. . . . . . . . . . 36

2.2.2 Multimode solitons . . . . . . . . . . . . . . . . . . 37

2.2 .3 MM soliton energy . . . . . . . . . . . . . . . . . . . 38

2.2.4 Spatiotemporal solitons in MMF versus MM solitons . . . 44

2.3 Spatiotemporal fission and Raman scattering of multimode solitons 45

2.3.1 Observations of multimode solitons . . . . . . . . . . 48

2.4 Spatiotemporal oscillations of multimode solitons . . . . . . . . 51

2.4.1 Oscillation instability of solitons (including MM solitons) 52

2.4.2 Cataloguing the instabilities of MM solitons and waves in GRIN fiber . . . . . . . . . . . . . . . . . . . . 54

2.5 Complex nonlinear optics: controlling the bewildering range of nonlinear effects . . . . . . . . . . . . . . . . . 57 


\section{Self-organization and spatiotemporal instability in normal-dispersion}

multimode fibers $\quad 59$

3.1 Nonlinear beam self-cleaning in multimode graded-index fiber . 60

3.2 Integrating the role of disorder and dissipation . . . . . . . . 62

3.2.1 Coupled mode theory for describing the network . . . . . 64

3.2.2 Application to beam self-cleaning with disorder and dis-

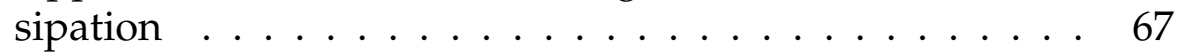

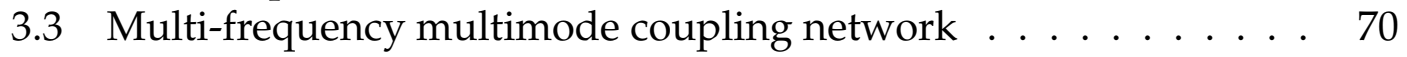

3.3.1 Geometric parametric instability . . . . . . . . . . . . . 71

3.3.2 Spatiotemporal modulation instability . . . . . . . . . 71

3.4 Self-organized instability . . . . . . . . . . . . . 72

4 Spatiotemporal mode-locking and multimode fiber lasers $\quad 75$

4.1 Introduction . . . . . . . . . . . . . . 75

4.1.1 Longitudinal and transverse modes in laser cavities . . . . 76

4.1.2 Spatiotemporal mode-locking . . . . . . . . . . . . . 79

4.1.3 Understanding STML with a first example . . . . . . . . 86

4.2 First experimental realization . . . . . . . . . . . . . 91

4.3 Fully-multimode fiber cavity $\ldots \ldots \ldots \ldots \ldots$

4.3 .1 Justifying the model . . . . . . . . . . . . . . . . . 95

4.3.2 Experimental and numerical results in cavities with multimode gain media . . . . . . . . . . . . . 97

5 Future directions 106

5.1 Advances in MMNLFO experimental methods . . . . . . . . . . 107

5.2 Highly multimode nonlinear waves in multi-pass cells, passive resonators and multi-layer media . . . . . . . . . . . . . . . 110

5.3 Complex nonlinear optics . . . . . . . . . . . . . . . . . . 112

5.4 Controlling light propagation in 4D: spatiotemporal nonlinear optical 'walks' and routing . . . . . . . . . . . . . . . . . . 115

$\begin{array}{ll}\text { Bibliography } & 117\end{array}$ 


\section{LIST OF FIGURES}

1.1 Multimode optical fibers. . . . . . . . . . . . . . . . . . . 14

1.23 dimensions of the world of MM nonlinear optical pulse propagation. . . . . . . . . . . . . . . . . . 27

1.3 Prototype of the experimental setup for MMNLFO experiments in Chapters 2-3. . . . . . . . . . . . . . . . . . 28

2.1 Propagation in anomalous dispersion GRIN MMF. . . . . . . . 34

2.2 Linear MM propagation in spacetime. . . . . . . . . . . . . . 35

2.3 Multimode solitons in spacetime. . . . . . . . . . . . . 38

2.4 Theoretical guess at the factor modifying the energy-area theorem for multimode solitons compared to a soliton in the fundamental mode. . . . . . . . . . . . . . . . . . . . . . . 41

2.5 Direct observation of a few-mode Raman-shifted MM soliton. . . 49

2.6 Changing $A_{\text {eff }}$ of the Raman-shifted soliton for increasing soliton energy. . . . . . . . . . . . . . . . . . 50

2.7 Mode structure of a parabolic potential. . . . . . . . . . . . . . 51

2.8 Summary of controlling nonlinear effects and spatiotemporal supercontnuum in GRIN MMF. . . . . . . . . . . . . . . . . 58

3.1 Kerr beam cleaning of femtosecond pulses. . . . . . . . . . . . . 61

3.2 Experimental observation of self-organized instability in disordered, dissipative multimode graded-index fiber. . . . . . . . . 63

3.3 Spatiotemporal instability in multimode graded-index fiber. . . . 65

3.4 Effect of disorder on self-organized energy flow into lower-order modes in GRIN fiber. . . . . . . . . . . . . . . . . . . . . 70

3.5 Spatiotemporal instability of different field distributions in GRIN fiber. . . . . . . . . . . . . . . . . . . . . . 74

4.1 Examples of 3D modes of a multimode cavity. . . . . . . . . . 78

4.2 Conceptual depiction of lasing frequencies in a multimode laser cavity. . . . . . . . . . . . . . . . . . . . 80

4.3 Examples of spectra of STML lasers. . . . . . . . . . . . . . 83

4.4 Conceptual depiction STML laser cavity. . . . . . . . . . . . . 85

4.5 Numerical example of STML in multimode fiber laser. . . . . . . 88

4.6 Intracavity evolution of the numerical STML example. . . . . . . 90

4.7 Schematic of the first, simplified MM laser cavity. . . . . . . . . . 92

4.8 Experimental and theoretical results in the first cavity. . . . . . . 93

4.9 Slow-fast dissipative soliton, numerical example. . . . . . . . . . 99

4.10 Example of different types of STML states observable by slowly changing one parameter of the system. . . . . . . . . . . 103

4.11 Experimental example of different types of STML states observable by slowly changing one parameter of the system. . . . . . 105 
5.1 New approaches for better measurement and control of MMNLFO experiments. . . . . . . . . . . . . . . . . . . . 108

5.2 Multi-pass cells and resonators as flexible platforms for extreme nonlinear light sources and spatiotemporal nonlinear optical pulse propagation. . . . . . . . . . . . . . . . . 111 


\subsection{Foreword: a guide to this thesis}

At the time of writing, the standard approach to a scientific PhD falls somewhere between literally stapling the student's papers together and writing entirely new material to document at least the main course of research undertaken at least primarily by the PhD candidate. I see nothing wrong with either approach, although a compromise between the two - especially when the published record of the work is especially sparse - has merits for all the retrospective perspective and cohesion that often eludes hot-of-the-presses science. Furthermore, the candid detail of the latter style has often been immensely valuable to me, so much so that I have attempted to incorporate this level of transparency and earnest reproducibility in my published works (exploiting the supplementary information many journals allow), as well as even further in works outside traditional peer reviewed journals, such as videos, open-source code and tutorials.

So preamble aside, this thesis is essentially in two parts, and the part you are reading now is an attempt to distill down that retrospective perspective and conceptual cohesion into a manageable document. It has been somewhat successful, and I hope my committee will be interested in discussing their assessment of its value in this regard - it is really an experiment in modern thesis writing in the natural sciences.

The second part is the 'stapled papers', which are listed below. For the most part, in this sequence these 'stapled papers' form a relatively self-contained thesis document. The intended audience for this 'stapled' thesis is, roughly speaking, researchers in nonlinear optical pulse propagation and lasers, although my 
hope is that much of the work is accessible more broadly.

Likewise, this thesis (the one you are reading now) is meant to be a selfcontained thesis document. The intended audience for this thesis is primarily future students in Frank's research group, or entering other groups working on this topic, and secondarily researchers (including my committee) who may be interested in a relatively big picture account of what is an intricate and involved body of research, and who may also appreciate this document's relatively short length (compared to the 500-600 pages the 'stapled' version comprises in this format). Accordingly, I am focusing here on the core concepts, the recurring themes and the fundamental motivations of the work in the 'stapled' thesis. Additionally, a core deficiency of the 'stapled' thesis is that it amounts to a continuous description of what we (the authors) thought at the time. With the benefit of time and deeper understanding, none of the conclusions turn out to be incorrect. That said, often the essential features are more clear.

In addition, in parts of this thesis I have taken a colloquial approach to recalling the research endeavor. My motivation for this is emphatically not that the research and the process of carrying it out has been exceptionally unusual in this regard, or especially dramatic. While I have found the adventure of exploring the multimode nonlinear optical hyperspace exciting, the human side of the story lacks the political intrigue, and drama of most stories. Rather, my intent is to convey especially to novice scientists (a group I still count myself a part of) the human dimension, the weakly-biased random walk of research.

My approach to references here is also deliberately selective, and my intent is that the bibliography contains a list of works that almost all qualify as 'mustread' for someone interested in performing research in this area. The 'must- 
read' are just the starting point however, and beyond that I encourage readers to examine the more complete references of Ref. [1] and [2].

Finally, an additional way to view these theses is that each chapter here is meant as a kind of introduction (and retrospective commentary/summary) for the corresponding 'chapter' in the stapled thesis, something to be read before the stapled chapter.

\section{Introduction}

Sections A-C and F from: L.G. Wright, Z.M. Ziegler, P.M. Lushnikov, Z. Zhu, M.A. Eftekhar, D.N. Christodoulides, and F.W. Wise (2018) Multimode Nonlinear Fiber Optics: Massively Parallel Numerical Solver, Tutorial and Outlook, IEEE Journal of Selected Topics in Quantum Electronics 24 (3), 1-16. This tutorial is focused on modeling nonlinear pulse propagation in passive multimode fiber using the generalized multimode nonlinear Schrödinger equations (GMMNLSE). This discussion is primarily complementary to this thesis, which focuses instead on the spatiotemporal model, the 3+1 D generalized NLSE.

\section{Multimode optical solitons}

L.G. Wright, W.H. Renninger, D.N. Christodoulides, and F.W. Wise (2015) Spatiotemporal dynamics of multimode optical solitons, Optics Express 23 (3), 34923506.

L.G. Wright, D.N. Christodoulides, and F.W. Wise (2015) Controllable spatiotemporal nonlinear effects in multimode fibres, Nature Photonics 9 (5), 306-310.

L.G. Wright, S. Wabnitz, D.N. Christodoulides, and F.W. Wise (2015) Ultrabroadband Dispersive Radiation by Spatiotemporal Oscillation of Multimode Waves, Physical Review Letters 115 (22), 223902.

Z. Zhu, L.G. Wright, D.N. Christodoulides, and F.W. Wise (2016) Observation of multimode solitons in few-mode fiber, Optics Letters 41 (20), 4819-4822. 


\section{Self-organization and spatiotemporal instability in multimode fibers}

L.G. Wright, Z. Liu, D.A. Nolan, M.-J. Li, D.N. Christodoulides, and F.W. Wise (2016) Self-organized instability in graded-index multimode fibres, Nature Photonics 10, 771776, including the supplementary material.

Z. Liu, L.G. Wright, D.N. Christodoulides, and F.W. Wise (2016) Kerr self-cleaning of femtosecond-pulsed beams in graded-index multimode fiber, Optics Letters 41 (16), 3675-3678.

\section{Spatiotemporal mode-locking and multimode fiber lasers}

L.G. Wright, D.N. Christodoulides, and F.W. Wise (2017) Spatiotemporal modelocking in multimode fiber lasers, Science 358 (6359), 94-97, including the supplementary material.

\section{Future directions}

W. Fu, L.G. Wright, P. Sidorenko, S. Backus, and F.W. Wise (2018) "Several new directions for ultrafast fiber lasers," Optics Express This prospective paper outlines recent work during my PhD, and a large number of ways forward. The focus is on ultrafast source development, with the last section being devoted to multimode fiber lasers.

Section G from L.G. Wright, Z.M. Ziegler, P.M. Lushnikov, Z. Zhu, M.A. Eftekhar, D.N. Christodoulides, and F.W. Wise (2018) Multimode Nonlinear Fiber Optics: Massively Parallel Numerical Solver, Tutorial and Outlook, IEEE Journal of Selected Topics in Quantum Electronics 24 (3), 1-16.

6. Appendix 1: Code for solving the generalized multimode nonlinear Schrödinger equations

Sections D-E from L.G. Wright, Z.M. Ziegler, P.M. Lushnikov, Z. Zhu, M.A. Eftekhar, D.N. Christodoulides, and F.W. Wise (2018) Multimode Nonlinear Fiber Optics: Massively Parallel Numerical Solver, Tutorial and Outlook, IEEE Journal of Selected Topics in Quantum Electronics 24 (3), 1-16. 
Documentation for GMMNLSE codes, available online with the MATLAB code itself at: https://github.com/WiseLabAEP/GMMNLSE-Solver-FINAL

L.G. Wright, Z.M. Ziegler, Z. Zhu, P.M. Lushnikov, and F.W. Wise, "Advanced Examples of Use of the GMMNLSE Solver", included as additional documentation and available online at the address above.

7. Appendix 2: Code for solving the generalized multimode nonlinear Schrödinger equations with gain, and the generalized (3+1-D) nonlinear Schrödinger equation

Z.M. Ziegler (2017), "Numerical tools for optical pulse propagation in multimode fiber", BSc. Thesis, Cornell University. Zach developed MATLAB codes for modeling ultrafast fiber lasers, amplifiers based on multimode fiber (with my supervision). His thesis is an excellent introduction to the theoretical bases for these codes.

Documentation for the 'MM Tools' codes, available with the MM Tools MATLAB code package. 
CHAPTER 1

INTRODUCTION

\subsection{Motivations}

Multimode nonlinear fiber optics (MMNLFO) spans dimensions. It spans space and time, order and chaos. It even spans ideology.

These are, admittedly, fairly whopping if not legitimately silly statements. Whether you know about MMNLFO or not, MMNLFO sure seems like a niche topic. It is. After all, it is an area (multimode nonlinear fiber optics) within a topic (nonlinear fiber optics) within a subfield (fiber optics) within a subfield (optics) of a discipline (physics) of science. Nonetheless, by the end of this chapter you should understand why these surprising statements are at least plausible. By the end of this thesis, you may even agree that they are true...

What do we mean by 'ideology'? To be very brief about it, we mean the system of beliefs and understanding, the 'lens' through which we as a society of people doing science care about and retrieve contextual meaning from our scientific endeavors. And often more importantly, how the society we are part of cares (or doesn't care) about our scientific endeavors. We can categorize relevant ideologies as (1) curiosity-driven science/'pure' science, (2) humanist science/technology, (3) militaristic or nationalistic science/technology, and (4) economically-motivated science/technology. The first refers to 'basic research', the discovery of how nature is and how it works, expressly for no particular purpose other than to understand. The second refers to science or technological development that may improve the human condition (usually by some measure 
of wellness like Maslow's hierarchy). The third refers to science or technology development that gives the nation doing it an advantage either in war or avoiding war. Finally, the last refers to science or technology development which has the potential to provide some kind of economic capital like money. Today, subscription to the second and fourth ideologies can be virtually taken for granted, and this encompasses the majority of scientific research. Nonetheless, categories (1) and (3) are popular (and routinely relevant) as well.

The thing to consider here is that these ideologies essentially define the (majority of) measures by which modern humans, as a collective societal group and over relatively long timescales, attempt to prioritize what science should be done. Of course, this attempt is only ever partially successful, but over sufficiently long timescales we might assume that fluctuations like fashions, fads, peculiar funders or individuals tend to average out, leaving these core ideological principles for prioritizing.

Obviously, most scientific endeavors span these ideologies, having some relevance toward them all (and others, even). Nonetheless, MMNLFO certainly does, and to such an unusual extent that it is worth starting by formally acknowledging this as a basic motivation, or argument, for its study.

First, and most importantly, MMNLFO is promising for advancing basic scientific tools, through which it has a 'secondhand' chance at very widespread impact encompassing all the ideologies just mentioned. This is probably the mode of impact that optics and optical physics has been most successful with, as measurements, manipulations and other tools based on light have been key to countless major developments throughout science. The impact of tools like the telescope, the microscope, the interferometer, the spectrometer, etc. are easy 
to take for granted. In the technology sphere (humanist science impacts), two similarly under-appreciated infrastructural elements are optical lithography, the enabler of modern mass-manufactured integrated electronics, and optical fiber, the physical basis for the internet. Today, in both scientific and technological applications, the laser is becoming just as easily taken for granted. Yet, whatever year it may be that you are reading this sentence, chances are at least one of the new Nobel laureates relied on one to make their seminal discovery.

As will be elaborated on later in this chapter, and particularly in Chapter 4, the application of MMNLFO to ultrafast lasers is promising as a means of creating compact, cost-efficient ultrafast lasers. These might be used by scientists in many disciplines, and could perhaps make rare, specialized and expensive state-of-the-art techniques into tools almost as ubiquitously mainstream as microscopes, telescopes and spectrometers. Ultrafast lasers can form the engine, for example, for volumetric biomedical imaging[3], surgical guidance and rapid diagnosis[4], or various applications in prototyping and manufacturing $[5,6]$. These same lasers might, as the engine of a more complex instrument involving high-harmonic generation of coherent $x$-rays $[7,8]$ or acceleration of charged particles in dielectric accelerators[9], allow for similar mainstreaming of technologies like synchotron-like radiation[8], electron-like microscopy[7] and more. Similar kinds of 'ideological transcendence' arguments apply to applications of MMNLFO in telecommunications or information processing $[10,11,12]$ which will be expanded both later in this chapter as well as in the last one. Finally, the impact of MMNLFO-based scientific tools in basic research is especially exciting if we consider the application of MMNLFOinspired ideas to solid-state laser technology, which (as described in the ultimate chapter of this thesis), could provide a means of building the highest peak 
power lasers, with the strongest human-made electric fields ever.

Second, MMNLFO is a scientific sandbox, full of emergent phenomena and dense with conceptual connections to other fields. This is another bold statement for sure - not one I expect you to agree with just yet. However, as we develop the conceptual framework for MMNLFO, and examine results of first forays into it, I hope you will slowly see that MMNLFO encompasses nonlinear physics that can, through relatively simple theoretical and experimental methods, span a bewildering hyperspace of situations and phenomena. It is therefore a prime place to study the behavior of light, and especially intense pulses of light. Through mathematical analogies and other conceptual cross-fertilization, it is possible that ideas or discoveries from MMNLFO might even impact more distant fields as well.

Now, even with the evidence and arguments put forward in this thesis, it should be acknowledged that this second mode of broader intellectual impact seems less likely than the first. However, by its very nature the impact of this category of science is hard, if not fundamentally impossible, to predict. Who knows what impact (in all ideological senses and otherwise) will come from discovering things we previously did not know? 


\subsection{The theoretical basics of multimode nonlinear fiber optics}

\subsubsection{The 3+1 D generalized nonlinear Schrödinger equation}

Everything you need to know about multimode nonlinear fiber optics ${ }^{1}$ is encapsulated (or at least can be added with additional, simple terms) in Eqn. 1. The best name for Eqn. 1 is the $3+1$ dimensional generalized nonlinear Schrödinger equation (3+1 D GNLSE), although it is closely related to the GrossPitaevskii equation[14], the 3+1-D Schrödinger equation, and really any other high-dimensional wave equation.

$$
\partial_{z} A(x, y, t ; z)=\frac{i}{2 \beta\left(\omega_{o}\right)} \nabla_{T}^{2} A-i \frac{\beta_{2}}{2} \partial_{t}^{2} A+i \frac{\beta\left(\omega_{o}\right)}{2}\left[\left(n(x, y, z) / n_{o}\right)^{2}-1\right] A+i \frac{n_{2} \omega_{o}}{c}|A|^{2} A
$$

Here, $\beta\left(\omega_{o}\right)=n_{o} k=n_{o} \omega_{o} / c$ is the propagation constant, at the center frequency, where the refractive index $n_{o}$ is typically taken to be the index at the center of the waveguide, or the average about which inhomogeneities of $n(x, y, z)$ occur. $\partial_{t}^{2}=\frac{\partial^{2}}{\partial t^{2}}$, and $\nabla_{T}=\partial_{x}^{2}+\partial_{y}^{2}$ is the Laplacian for the transverse coordinates ( $x$ and $y$, being orthogonal or transverse to the 'long', or 'evolution', coordinate $z$ ). $\beta_{2}$ is the group velocity dispersion of the medium, $\partial_{\omega}^{2} \beta$, evaluated at $\omega_{o}$. Finally, $n_{2}$ is the nonlinear refractive index of the medium.

The left hand side (LHS) and first two terms on the right hand side (RHS) of Eqn. 1 comprise a 3D wave equation with the paraxial approximation and slowly-varying envelope (SVEA) approximations having been made. Here,

\footnotetext{
${ }^{1}$ Particularly in Chapters 2-3, I am assuming readers have a basic familiarity with concepts of single mode nonlinear fiber optics, outlined in Ref. [13].
} 
$A(x, y, t ; z)$ is the electric field envelope (that is, the carrier wave, with angular frequency $\omega_{o}$, has been factored out $[13,15]$. The two approximations correspond to the assumption that we are interested in waves that are mostly pointed parallel to the ' $z$ ' axis, and that the bandwidth of the pulse represented by $A(x, y, t ; z)$ at any point along $z$ is small compared to the center frequency $\omega_{o}$. In fiber optics, the paraxial approximation is almost always valid provided the numerical aperture of the fiber is much less than 1, and the SVEA is also true for most of the situations considered in this thesis ${ }^{2}$. We are also assuming implicitly that the field is propagating in one direction along $z$. Multiple polarizations can be easily accounted for by solving a system of two equations, coupled by the nonlinearity, with possibly different values of $\beta$ (if the medium is birefringent) and relative velocity [13].

Assuming you have familiarity with the mathematical machinery of entrylevel quantum mechanics, an analogy will help you if this is your first time seeing this equation ${ }^{3}$, by relating back to its namesake, the linear Schrödinger equation (LSE). In the LSE, the equation describes the time evolution of the wave function $\Psi(x, y, z ; t)$, the magnitude-squared, $|\Psi(x, y, z ; t)|^{2}$ which corresponds to the probability of finding the particle at each location in 3D space at time $t$ (the probability density). The NLSE here describes the 'evolution' (or 'beam propagation') of a localized pulse of light in spacetime, $A(x, y, t)$ along the $z$-coordinate, where the units of $A$ are chosen such that $|A(x, y, t)|^{2}$ is the intensity per unit area (or energy density in spacetime, $J / s / \mathrm{m}^{2}$ ). Finally, Eqn. 1 is in the

\footnotetext{
${ }^{2}$ With respect to the SVEA, NLS-type equations are known to be surprisingly accurate even way beyond where they should according to the SVEA. This is because the same, or nearly the same, equation can be derived without actually making the SVEA, but instead by making an assumption that is often implicit in derivations, that of unidirectional propagation along the $z$-axis [16].

${ }^{3}$ In fact it may help you later on as well, since many modern research themes in optics, such as Anderson localization[17] and PT-symmetry[18, 19] rely on the paraxial wave equation and Schrödinger equations being the same.
} 
reference frame of the pulse moving at the group velocity of the medium at the center frequency. In this reference frame, 'positive time' happens after whatever happens at $t=0$, so for example the part of a pulse that is at the most-positivetime-edge is often referred to as the 'trailing edge'.

\subsubsection{Modes}

If we add the third term, $i \frac{\beta\left(\omega_{o}\right)}{2}\left(\left(n(x, y, z) / n_{o}\right)^{2}-1\right) A$, to the 3D paraxial/SVEA wave equation, we have the ingredients for modes. For simplicity and relevance, let's suppose we have a monochromatic wave, so the time domain is trivial (unchanging, so it factors out and the terms on the RHS that involve it are zero), and that $n(x, y, z)=n(x, y)$ (which is the case in an ideal optical fiber), so the equation can be written as:

$$
\partial_{z} A(x, y ; z)=\frac{i}{2 k_{e}\left(\omega_{o}\right)} \nabla_{T}^{2} A+i \frac{k_{e}\left(\omega_{o}\right)}{2}\left(\left(n(x, y) / n_{o}\right)^{2}-1\right) A
$$

Much as you might in quantum mechanics, you can find eigenfunctions of this equation, that satisfy the following:

$$
\partial_{z} A_{p}(x, y ; z)=\frac{i}{2 \beta\left(\omega_{o}\right)} \nabla_{T}^{2} A_{p}+i \frac{\beta\left(\omega_{o}\right)}{2}\left(\left(n(x, y) / n_{o}\right)^{2}-1\right) A_{p}=\hat{H} A_{p}=i \beta_{p} A_{p}
$$

In general, a given waveguide, defined by the optical potential $n(x, y)$, has a number of modes, $F_{p}(x, y)$, which are an orthonormal, incomplete set, and each of which has a distinct propagation constant $\beta_{p}$, analogous to the different energy or frequency of eigenfunctions in a typical quantum mechanical 
potential[20]. It is common for there to be groups of modes in the waveguide that, due to some symmetry of the fiber, are degenerate, or at least quasidegenerate, in the sense that in an ideal, birefringence-free fiber, the propagation constants are exactly the same. For example, the LP11 mode has two versions, related to each other through a 90 degree rotation (which is obviously a symmetry of the cylindrical ideal fiber, which is symmetric for any rotation). Note that, like any potential in quantum mechanics, beyond the 'bound states' of the modes, there exists a continuum of modes. In optics, these are referred to as radiation modes, since they radiate away from the fiber. In fiber optics, before reaching the radiation modes, one actually has 'cladding modes', since the cladding of the fiber, itself being clad by a polymer, air, or some other medium, comprises a very multimode optical fiber. Finally, note that here and throughout we are ignoring the polarization, the vectorial nature of the field. For linearlypolarized fields in short, weakly-guiding MMFs this is reasonable approach, especially in the interest of keeping the description as simple as possible.

There are many types of optical waveguides to consider, but for MMNLFO, the most important are probably the parabolic graded-index (GRIN) fiber and the step-index fiber, shown in Figure 1.

How many modes does a waveguide support? To understand why a given waveguide has the number of guided modes it has, we introduce some basic Fourier transform relationships ${ }^{4}$. First, the Fourier transform of $A$ in time gives us $A(x, y, \omega)$, which is a potentially spatially-varying function whose magnitude squared, $|A(x, y, \omega)|^{2}$ could be called the power spectral density per unit area. A spectrometer with a very small input aperture (such as a single-mode fiber) may

\footnotetext{
${ }^{4}$ It is worth being a little careful about Fourier transform definitions, which in optics are often the complex conjugate of the conventions that are used in other fields or that are assumed, e.g., by MATLAB or other math packages.
} 
(a)

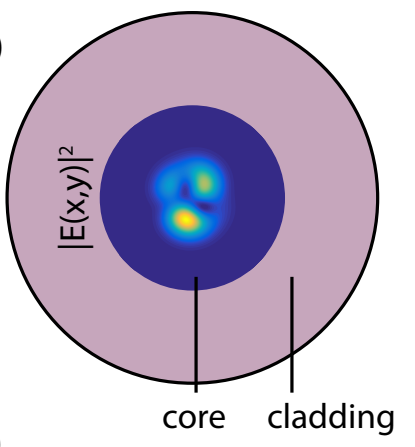

(c)

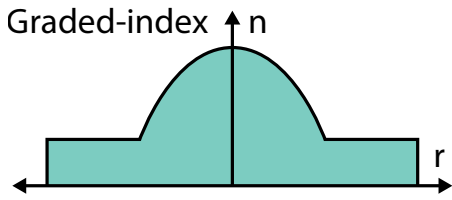

Step-index

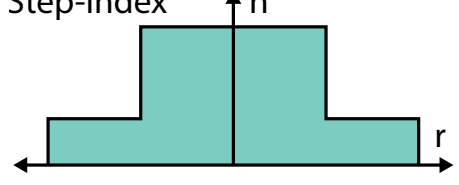

(b)
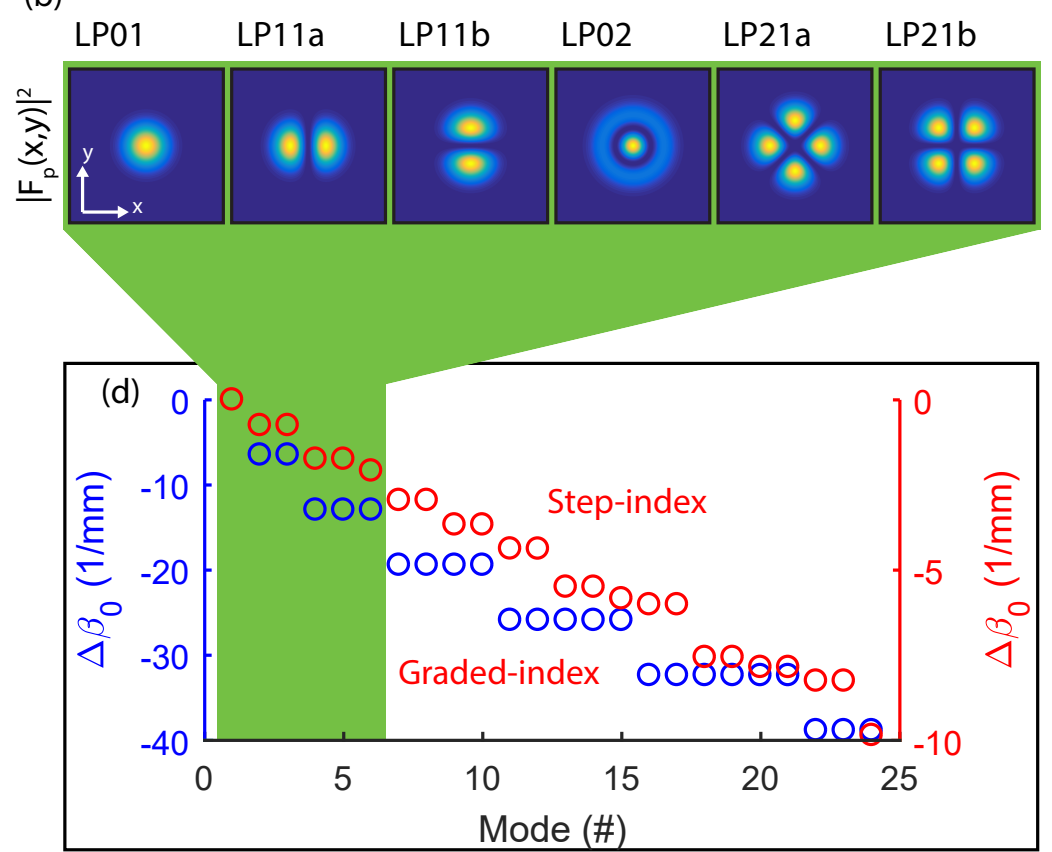

Figure 1.1: Multimode optical fibers. (a) shows a random superposition of the first 6 modes of a graded-index fiber. The modes are shown in (b). (c) shows the index profile of graded-index (GRIN) and step-index fibers. (d) shows the distinct propagation constants for a GRIN fiber and step-index fiber of the same size and with the same center to cladding index difference (numerical aperture).

measure something close to $|A(x, y, \omega)|^{2}$, while if the entire field is coupled into the spectrometer, it measures something closer to $\int_{-\infty}^{\infty} \int_{-\infty}^{\infty}\left|A\left(x^{\prime}, y^{\prime}, \omega\right)\right|^{2} d x^{\prime} d y^{\prime}$.

If we instead take a two-dimensional Fourier transform along the transverse coordinates, we obtain $A\left(k_{x}, k_{y}, \omega\right)$, which is the field in ' $k$-space'. Here, the $k_{(x, y)}$ are the spatial frequencies of the field. In the paraxial approximation, ' $k$-space' is virtually equivalent to a more intuitive 'angle from $z$ space' obtained by dividing $k_{(x, y)}$ by the magnitude of the total wavevector, $n_{o} \bar{k} \approx n_{o} k_{z}=\beta$, so that the transverse coordinates are $\theta_{(x, y)} \approx k_{(x, y)} / \beta$. In this case, $\left|A\left(\theta_{x}, \theta_{y}, \omega\right)\right|^{2}$ can be interpreted as the distribution of ray trajectories at each value of $\omega . A(x, y)$ is referred to as the 'near-field', while its transverse spatial Fourier transform is the 'far-field'. 
The number of modes a given optical potential has is essentially the product of its 'area' in $k$-space, or the far-field, and its actual area in 'real-space', or the near-field. For parabolic GRIN fiber, including both polarizations, the number of modes is [21].

$$
M=\frac{1}{2} \beta^{2} R^{2} \frac{n_{o}-n_{\text {cladding }}}{n_{o}}
$$

For quite multimode (large $M$ ) step-index fibers, an approximate similar expression exists,

$$
M \approx \frac{1}{2} \beta^{2} R^{2} \frac{n_{o}^{2}-n_{\text {cladding }}^{2}}{n_{o}^{2}}
$$

where in both cases, $n_{o}$ refers to the refractive index at the center of the fiber.

If we slowly vary the center frequency in Eqn. 1.3, we will obtain a differentbut-similar set of modes, with different-but-similar propagation constants. The latter define independent functions $\beta_{p}(\omega)$. As is evident from the previous two equations, the number of modes depends on the frequency (through $\beta(\omega)=$ $n(\omega) \omega / c$, as well as clearly through $n(\omega))$. So, for the very highest-order modes whose propagation constants are close to the propagation constant of waves in the cladding $\left(\beta_{\text {cladding }}=n_{\text {cladding }}(\omega) \omega / c\right)$, and whose field distribution $A_{p}(x, y)$ involves a lot of energy very close to edges of the core, changing the frequency leads to very large changes in $\beta_{p}$ and $A_{p}(x, y)$. In fact, while $\beta_{p}$ is a real (or at least mostly real) number for the guided modes, modes near their 'cut-off frequency' develop a large imaginary component, which corresponds to very high propagation losses due to radiation. Assuming we are not dealing with such modes, we can usually safely expand $\beta_{p}(\omega)$ as a Taylor series about $\omega_{o}$. From 
here on, we will adopt a notation for the $n$-th order coefficient of this Taylor series for each mode $p$ as $\beta_{n}^{(p)}$, so that the Taylor expansion is:

$$
\beta_{p}(\Omega)=\beta_{o}^{(p)}+\beta_{o}^{(p)} \Omega+\frac{1}{2} \beta_{2}^{(p)} \Omega^{2}+\frac{1}{6} \beta_{3}^{(p)} \Omega^{3}+\ldots
$$

where $\Omega$ is $\omega-\omega_{o}$.

\subsubsection{The multimode nonlinear Schrödinger equations}

Armed with this knowledge, and recalling that the modes are orthogonal, we can perform a change-of-basis operation to Eqn. 1.1 from 'real space' ( $x$ and $y$ ) to 'mode space'. When we do this, it is convenient to choose a reference frame at the center frequency in one of the modes. This amounts to subtracting the global longitudinal phase evolution, and the group velocity of that mode. This is typically mode 1 , so the frame's phase and group velocity are $\beta_{o}^{(1)}$ and $\beta_{o}^{(1)}$ respectively. This procedure produces the multimode nonlinear Schrödinger equations (MMNLSE)[22, 23, 1]:

$$
\partial_{z} A_{p}(z, t)=i \delta \beta_{0}^{(p)} A_{p}-\delta \beta_{1}^{(p)} \partial_{t} A_{p}+\sum_{m=2}^{N_{d}} i^{m+1} \frac{\beta_{m}^{(p)}}{m !} \partial_{t}^{m} A_{p}+i \frac{n_{2} \omega_{o}}{c} \sum_{l, m, n}^{N} S_{p l m n}^{K} A_{l} A_{m} A_{n}^{*}
$$

where, assuming all real values, the relative propagation constant of mode $p$

is $\left(\delta \beta_{0}^{(p)}=\beta_{0}^{(p)}-\beta_{0}^{(1)}\right)$, the relative group velocity of mode $p$ is $\left(\delta \beta_{1}^{(p)}=\beta_{1}^{(p)}-\beta_{1}^{(1)}\right)$, and higher-order chromatic dispersions (group velocity dispersion, third-order dispersion, etc.) are included in the third term. For linearly-polarized modes 
whose scalar field distribution are given by $F_{p}(x, y)$, the Kerr coupling tensor is given by ${ }^{5}$ :

$$
S_{p l m n}^{K}=\frac{\int \mathrm{d} x \mathrm{~d} y\left[F_{p} F_{l} F_{m} F_{n}\right]}{\left[\int \mathrm{d} x \mathrm{~d} y F_{p}^{2} \int \mathrm{d} x \mathrm{~d} y F_{l}^{2} \int \mathrm{d} x \mathrm{~d} y F_{m}^{2} \int \mathrm{d} x \mathrm{~d} y F_{n}^{2}\right]^{1 / 2}}
$$

Now, it is not necessary to start our change-of-basis from Eqn. 1.1. We do not need to make the paraxial approximation to find the transverse eigenmodes. Thus, in practice it can be beneficial to find the modes of $n(x, y)$ without one or more of the approximations intrinsic to Eqn. 1.1. This is one major benefit of Eqn. 1.7.

The biggest advantage of the MMNLSE, however, is that, when the problem can be described with a small number of modes, the MMNLSE is much faster to solve numerically (as well as easier to interpret by humans). For typical solution methods and computers, 'small' means less than or equal to about 20 modes. As will be seen later in this thesis, even when the physics involves >> 20 modes, MMNLSE simulations involving a small subset of the modes can provide a good qualitative description, one that can at least form the basis for a physical understanding or for guiding more advanced simulations.

\subsubsection{The nonlinear term(s)}

The last term of Eqn. 1.1 and the many terms in the final sum of the MMNLSE represent the optical Kerr nonlinearity. Judging by the title of this thesis, these terms must be important. It is true that they are very important here, but it is

\footnotetext{
${ }^{5}$ See Ref. [23] for the general expressions, and Refs. [22] and [21] for more on additional corrections
} 
worth remarking that this is a very unusual situation for light. Since for fused silica $n_{2} \approx 2.3 \times 10^{-20} \mathrm{~m}^{2} / \mathrm{W}$, the term(s) are incredibly small unless the intensity $|A|^{2}$ is very high. However, this can easily be the case when dealing with femtosecond pulses in optical fibers.

If we consider just the nonlinear term from Eqn. 1.1, we have:

$$
\partial_{z} A(x, y, t ; z)=i \frac{n_{2} \omega_{o}}{c}|A|^{2} A
$$

From this, we can see several things. First, the nonlinear term modifies the phase of the field in space and time, and the amount it modifies the phase is larger where $|A|^{2}$ is larger. Recalling that $|A|^{2}$ is the intensity (in $\mathrm{W} / \mathrm{m}^{2}$ ), this can be interpreted as an 'intensity-dependent refractive index',

$$
n_{N L}(x, y, t ; z)=n_{o}(x, y ; z)+|A(x, y, t ; z)|^{2} A(x, y, t ; z)=n_{\text {linear }}(x, y, z)+n_{2} I(x, y, t ; z)
$$

Assuming $A(x, y, t)$ is a peaked function (like a Gaussian in time and space), this term acts a little like a lens. A lens' shape introduces an inhomogeneous phase front to an incident field which is largest at the center of the lens, and decreasing towards the edges. Another comparison is to a waveguide, which is sort of a very long lens that is never quite finished with the field. Like lenses or other inhomogeneous optical elements, the intensity-dependent refractive index modifies the phase of the field envelope $A$. But unlike lenses and waveguides made by inhomogeneous optics, the nonlinear index is self-generated, which means that, through the medium, light affects itself, which effects its propagation, which affects its effect on itself, etc. A nonlinear self-lens, for 
example, does not have a fixed focal length. Instead, as the field focuses and becomes more and more intense, the focal length of the nonlinear self-lens becomes shorter and shorter. If the total power of the beam exceeds a value, $P_{\text {crit }}$, which is about 4.5 MW in fused silica, then this process is catastrophic, and continues until the the beam 'collapses' into a singularity (which in reality means higher-order nonlinearities or spatiotemporal nonlinear-dispersive effects become relevant[24]).

This is the essence of nonlinearity. Especially in long fibers and/or with high intensity pulses, the cumulative effects of nonlinearity on the behavior and propagation of light are positively enormous, even when their effect at any given instant is rather small. This should be interesting enough. However, one additional difference between the nonlinear perturbation to the refractive index should be considered. While it is easy to imagine an optical component for which $n(x, y)$ varies in space, it is much more special to have such a variation in space-time. Something like this may be called spatiotemporal.

Now that you at least understand the title of this thesis, let us return to apply these concepts to understand in a little more detail, and with a little more specificity, why you should care (primarily assuming you subscribe to ideologies 1 and 3).

\subsection{More specific motivations}

If we understand multimode nonlinear fiber optics, a lot of things we'd like to scale can be scaled by at least $M$, the number of modes we can use. Here are a handful: 
1. The peak power from ultrafast fiber lasers

2. The information transmission capacity of optical fiber networks

3. The field of view in fiber endoscopes used for en vivo multi-photon or other nonlinear optical microscopies

4. The number of degrees of freedom we have to control coherent light

5. The number of nodes or elements in an optical computer, or the capacity of an optical information processing device.

Let us examine the first two in more detail.

\subsubsection{Peak power of ultrafast fiber lasers}

A mode-locked laser is a laser that can produce an extremely short $\left(\approx 10^{-13} \mathrm{sec}-\right.$ onds long) pulse. The peak power of the pulse from a mode-locked laser is determined by how short a pulse can be produced, and by the amount of energy which can stably be stored in the pulse. The limiting factor is the intensity of the pulse. Even though nonlinearity is required for a mode-locked pulse to form, too much nonlinearity limits the peak intensity. When the intensity, $I$, is too high, the nonlinear phase shifts (i.e., the cumulative effects of nonlinearity) within the laser cavity $\left(\phi_{N L} \sim n_{2} I L\right.$ for $L$ being related to the length of the cavity) are too large, a single pulse will either break apart into multiple sub-pulses, or will disintegrate into noise. Different types of mode-locked lasers encounter this limit for different amounts of nonlinearity, but all types can handle only so much. More intuitively, if the peak intensity is too high, eventually the laser medium will be damaged. 
The intensity, $I=P / A_{\text {eff }}$, is just the power $P$ divided by the cross-sectional area of the laser beam inside the medium, $A_{\text {eff }}$. For a fiber, $A_{\text {eff }} \sim \pi R^{2}$. Whether the fiber is step-index or GRIN, or whatever else, glancing at Eqns. 1.4 and 1.5 shows that $A_{e f f} \propto M$. Thus, for a laser operating at the maximum intensity possible (limited either by the nonlinearity or optical damage), $I_{\max }$, the power is clearly just proportional to $M, P_{\max } \propto A_{\text {eff }} I_{\max } \propto M$. In other words, the maximum power the laser can produce is proportional to $M$.

\subsubsection{Information capacity in optical fiber telecommunications}

In an earlier section, the reason that high-performance ultrafast fiber lasers matter was explained: they may enable a whole bunch of very useful scientific tools, in principle at accessible prices and in compact and even portable packages. If you believe that physical science has value, developing these tools is pretty close to self-evident. The case for better optical telecommunications may even be more self-evident, however. Today, economic activity and growth of all kinds is tied to the revolution of the internet, and demand for bandwidth is growing rapidly, and without any sign of slowing down [10,11,12]. If we can understand MMNLFO, we have the potential to increase the speed of the internet by 3-4 orders of magnitude, not to mention improve the value of future network scaling efforts. While compact ultrafast fiber lasers have the potential to accelerate much of physical science, MMF-based telecommunications have the potential to accelerate pretty much every modern human enterprise.

How can MMNLFO increase the speed of the internet exactly? While an ex-

act answer is beyond the scope of this thesis (and to the level of practical fact, 
remains to be proven), we can get some idea. In optical telecommunications, information is encoded on the phase and/or amplitude of short pulses of light. We can transmit a certain number of such pulses per unit time, limited by the total bandwidth of the optical fiber (that is, what range of wavelengths we can send through the fiber with low-loss. In practice this is set by the bandwidth of optical amplifiers.). The maximum information capacity through an optical fiber, or any communication channel for that matter, is set by the nonlinear interference between transmitted bits and the noise in the system[25]. In the fiber, the Kerr nonlinearity thus sets a fundamental limit for how much information we can transmit. In other words, we are stuck with $I_{\max }$ being the maximum average intensity of light within the optical fiber. Below $I_{\max }$, increasing the power of the transmitted signal improves the error-free signal capacity, since it improves the signal-to-noise ratio at the receiver. Beyond it, however, the capacity decreases or at least ceases to improve, due to the reduction of the signal-to-noise ratio by nonlinearity.

Now, when one reaches the limit with a given fiber, of course you can just add more fibers. This costs twice as much, however, and takes up twice as much space. But you could add modes to the fiber, using the additional modes for independent channels (so-called mode-division multiplexing, MDM, a simple version of space-division multiplexing, SDM). But on first glance, Eqns. 1.4 and 1.5 seem to show that adding twice as many modes also takes up twice as much space, since $M \propto A_{\text {eff }}$. So, if we already know how to do things with the singlemode fiber, we have nothing to gain by using MMFs, right?

But, the area of the MMF is much larger! So for a given power in the fiber, the intensity is lower, and thus we can send more signal power through the fiber 
before reaching the nonlinear limit.

Well, unfortunately this is not helpful either. While it is true that the area of a MMF is larger than a SMF, that a given mode channel has a higher nonlinear limit is only true if we are not using all the MMF's modes to transmit. If we use all $M$ modes as independent channels, operating at maximum capacity in each channel entails using $M$ times as much total power. Light in any given mode can interacts with the light in every other mode (through the many terms in the final sum of the MMNLSE). Thus, because the channels occupy the same area, the fact that they are larger does not obviously seem to improve the overall fiber transmission capacity.

Despite the failure of these two basic arguments to make the case for MMF in telecommunications, it turns out that there are two other arguments that do. First, single-mode fibers may have small cores, but the actual fiber diameter is typically around $125 \mu \mathrm{m}$. It cannot be much smaller than this before its mechanical strength becomes an issue. Thus, we can essentially increase the fiber core from $8 \mu \mathrm{m}$ for SMF up to about $100 \mu \mathrm{m}$, as well as increase the difference between the core and cladding index, to obtain many more modes 'for free' all without increasing the nominal cost or size of the transmission fiber. While there is a lot more to a fiber telecommunications line than the fiber [12], if all these modes were used, it would provide a healthy boost of the total capacity by nearly 4 orders of magnitude before the actual space taken up by the fiber increased.

The second argument for MMF in telecommunciations is that, even though the larger area does not help improve the nonlinear transmission limit because the number of modes scales as fast as the reduction in optical intensity, the 
nonlinear interference between different-mode channels, or within a multimode fiber may somehow be different from that in a single-mode fiber. While in general it is not known what the nonlinear limit for communications through a multimode optical fiber is, it is known in one special case - a special case that will nonetheless likely be relevant if SDM is widely implemented[26].

This result may be intuitively surprising, but it indicates that multimode fibers with $M$ modes which are affected by strong disorder have a transmission capacity that is higher than $M$ separate single-mode fibers. Using the $3+1 \mathrm{D}$ GNLSE and MMNLSE, we can develop an intuition for this result. Disorder inside the fiber amounts to fluctuations in the refractive index function that vary along $x, y$ as well as along $z$. Despite the randomnness, if the Kerr nonlinearity is excluded, the equation is still linear. This linearity means that it's solution (the effect of the fiber) across some distance $L$ can be written as a linear transmission matrix, $T(\bar{L})$, acting on the input field, so that:

$$
A(x, y, t, L)=\bar{T}(L) A(x, y, t, 0)
$$

If we are able to detect the electric field's amplitude and phase ${ }^{6}$, then we can characterize $T \overline{(L)}$ ahead of time. If the disorder is small and its average value is close zero ${ }^{7}$ then the fiber has $M$ guided modes, and $T(\bar{L})$ is completely represented by an $M \times M$ matrix which can be measured through $M^{2}$ total measurements. This means that, provided we do some pre-measurement of $T \overline{(} L)$, if it is fixed in time or slowly-varying, a MMF with disorder can be used for $M$

\footnotetext{
${ }^{6}$ Which is now commonly done in telecommunications: so-called 'coherent detection' has been a breakthrough technology for single-mode fiber transmission.

${ }^{7}$ That is $n(x, y, z)$ in Eqn. 1.1 can be written as $n(x, y)+\delta(x, y, z)$ where $\int_{\text {fibercore }} \int_{0}^{L} \delta(x, y, z) d z d A=$ 0 .
} 
independent channels, the eigenvectors of $\overline{T(L)}$.

Now, while the linear case seems to be effectively unchanged by disorder, the nonlinear situation is. Nonlinear interactions between modes (for present purposes, these are most clearly seen in the MMNLSE) can be categorized into those that depend on the phase of the modes, and those that depend only on the intensity, $\left|A_{p}\right|^{2}$. Interactions that depend on the phase correspond to terms that look, for example, like $A_{l} A_{m} A_{n}^{*}$, where $n \neq(l, m)$ in the MMNLSE's last sum. The disordered fluctuations of $n(x, y, z)$ cause the phases of each mode to evolve randomly along $z$. Critically, the different modes 'see' different parts of the random fluctuations, so their random phase evolutions are not correlated (more precisely, they are only correlated over short distances). The result of these random, uncorrelated phases, is that all such phase-sensitive terms average to zero. This has a huge effect. Instead of $M^{4}$ different nonlinear coupling terms, only $M^{2}$ remain after averaging out by random phase fluctuations. This corresponds to a dramatic reduction in the effect of the nonlinearity in causing transmission degradation by introducing interference between modes.

Of course, this rests on having a MMF with sufficiently strong disorder such that the above averaging condition is satisfied. Surprisingly, to some extent even conventional fibers satisfy this condition. Specifically, the disorder must decorrelate the phases of the fields in each mode faster than nonlinear phases accumulate. In conventional fibers, no natural birefringence exists, and instead randomly-distributed strain within the fiber causes random birefringence which most measures suggest is correlated over roughly $10 \mathrm{~m}$ [13]. Thus, provided the length scale over which nonlinear phase accumulates is $>>$ $10 \mathrm{~m}$, modes which are sensitive to this fluctuating birefringence are randomly 
perturbed. The good news is that, in optical telecommunications, this nonlinear length scale, which can be estimated by the so-called 'nonlinear length', $L_{N L}=A_{e f f} / n_{2} k P_{o}$, where $P_{o}$ is the peak power [13], will virtually always be $>>10$ $\mathrm{m}$ (remember, $n_{2}$ is a ridiculously small number). In GRIN fibers, as can be seen in Figure 1, modes fall into quasi-degenerate mode groups (this remains true as long as the waveguide is nearly parabolic and the index difference between core and cladding is relatively small). Modes within these groups are sensitive to random birefringence, so provided GRIN fibers are used and the condition $L_{N L}>10 \mathrm{~m}$ is met, to some extent the problem is solved. Of course, there is a bit more to it than this, but if you have understood everything up until now you can probably begin to guess some of the deeper details.

\subsubsection{Exploring the world of multimode nonlinear optical pulse propagation}

From a scientific perspective, while the numerous apparent potential applications of MMNLFO provide an initial argument for its study, MMNLFO represents a truly enormous space of optical physics. With a high degree of control in both experiments and theory/simulations, one can access different regimes of nonlinear wave propagation, or control specific features. An abstract representation of this world of MM nonlinear optical pulse propagation is shown in Figure 1.2.

This abstract space represents the terrain that can be mapped out by scientists studying MMNLFO, and served as something of a guide for the explo-

rations that make up this thesis. The early sections, Chapter 2, begin in the 


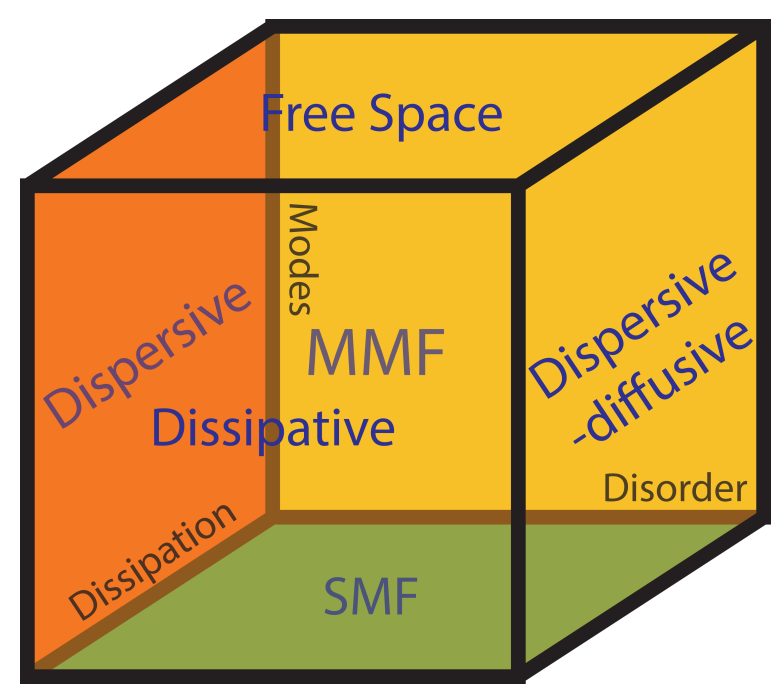

Figure 1.2: 3 dimensions of the world of MM nonlinear optical pulse propagation. By varying the number of modes, one may consider physics ranging from single mode $(1+1 \mathrm{D})$ to free space $(3+1 \mathrm{D})$ and in between. Varying disorder determines whether dispersion (due to modal dispersion and chromatic dispersion) are dominant, or if diffusive-like propagation is more relevant. Dissipation may likewise be varied, from being irrelevant to being a primary effect driving physics, such as in a MM laser. Other 'dimensions' could easily be added, such as mechanical coupling, 'quantumness' of the light involved, normal or anomalous dispersion, feedback or confinement in the $z$-dimension, and so on.

lower corner, furthest back in the interior of the cube: relatively few modes, and deliberately with negligible amounts of disorder and dissipation. The culmination of Chapter 2 is in observations of highly-multimode supercontinuum, which begins to exhibit features reminiscent of nonlinear optical pulse propagation in free-space. With subsequent studies, as in Chapter 3, we deliberately targeted a regime of MMNLFO where dissipation and disorder are important to the dynamics, and where a larger number of modes must be considered. This chapter, which is also set in the normal dispersion regime, reaches into the center of the cube. The fourth chapter takes us to the strongly-dissipative regime of the multimode fiber laser. Finally, the last chapter probes the remaining volume of the cube, as well as higher dimensions of the hyperspace of MM nonlinear dynamics. 


\subsection{Experiments in multimode nonlinear fiber optics}

Experiments in MMNLFO have, at least so far, been pretty simple. In the final chapter, we will visit our current and future projects that will trade a little of this simplicity for significantly enhanced experimental control and precision. However, to date the most important component of experiments in MMNLFO has been complementing it with thorough and careful theoretical and numerical studies, and where possible comparing predictions directly with the simple experimental measurements.

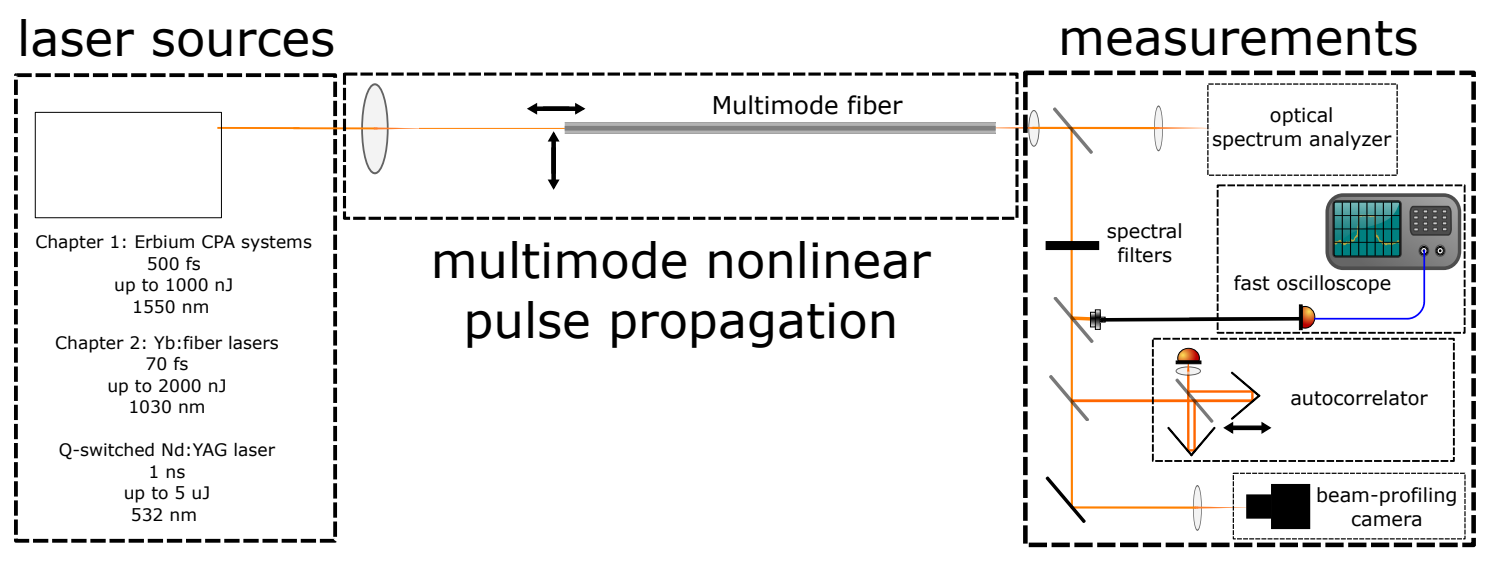

Figure 1.3: Prototype of the experimental setup for MMNLFO experiments in Chapters 2-3. High power laser pulses are launched into various multimode fibers, and the output is measured in many different ways, often using spectral filters to examine particular frequency regions independently.

That said, the simplicity of the MMNLFO experimental setup can be misleading. Yes, the primary techniques used in this thesis are fairly standard tools of ultrafast and nonlinear optics, such as the optical spectrum analyzer (OSA), the autocorrelator, and the beam profiling camera. Nonetheless, in using each of these devices, it has been very important to consider precisely what is actually being measured. Unless specific things are done to ensure otherwise, an OSA with a finite input aperture measures only a particular spatial location 
of a beam; for MM beams the spectrum may vary in space. The autocorrelator in general assumes a Gaussian beam, and especially if second-harmonic generation is employed, the signal depends on an assumed phase-matching between these beams. Using a phase-matching free alternative, like two-photon absorption [27], allows for spatially-integrated measurements. Alternatively, for sufficiently few-mode beams, or with spatial filtering beforehand, autocorrelation based on phase-matched second harmonic generation may be used to get a 'basic idea'. For beam profiling, one may need to employ achromatic lenses, and confirm the alignment of the imaging telescope for wavelengths across the beam. This is especially crucial when direct measurements are made to characteristic sizes of modes in the fiber - here a slight misalignment can dramatically affect the implication of a measurement.

\subsubsection{The 'mode-us' operandi of MMNLFO}

We began this chapter by talking about ideology. My intent (really, my experiment) has been for the thesis to embrace itself as a microcosm of science. Before getting purely into that microcosm, let us close the chapter by taking one final 'zoom out' to examine the context and guiding approach for the work subsequently summarized.

A number of measures, or general guidelines, have informed my approach. Obviously these are not unusual approaches in science, but for a number of reasons I think it is worth mentioning them briefly here. The primary reason is that this thesis is a microcosm of science, but the second is that I think it is easy for workers from either single-mode nonlinear fiber optics or from filamentation 
in free-space (pure $1+1$ or $3+1 \mathrm{D}$ respectively) to underestimate the thickness of the complexity of MMNLFO.

First, my efforts have focused on obtaining the broadest qualitative conclusions. The proper procedure has been to get as much experimental data as possible, with as many numerical simulations and theoretical approaches as possible, considering multiple hypotheses and in particular identifying the most unexciting 'null hypothesis' that could explain any given observation. What ultimately is reported in publications tends to be a select few experimental, theoretical and numerical simulation results that support this overall conclusion. I have (in the supplementary material usually), however, tried to make a habit of including also the most contrary data obtained, along with a comment on why this 'most contrary' data is still consistent with the overall conclusions.

Second, my focus has been on identifying core concepts: the tools that analytically, numerically and conceptually provide the most efficient or insightful description of the broadest qualitative conclusions. Solitons, or soliton-like waves, are one such concept, since they represent the stable 'nonlinear eigenmodes' of the complex nonlinear MM propagation. Nonlinear attractors, configurations of the field in space-time or particular trajectories of the field in phase space, whether they are soliton-like or not, are equally important. Instabilities are examples of non-soliton nonlinear attractors: they are also nonlinear attractors, just usually toward things not quite so aesthetically beautiful or useful as the solitons.

Finally, I have required that any major conclusion be supported by one or more types of numerical simulations, one or more theoretical analyses, and one or more sets of experiments (as well as obviously not be invalidated by any of 
the three). For example, if a conclusion is replicated by simulations involving the GMMNLSE and the 3+1 D GNLSE, it obtains more confidence. It is likewise of interest to observe whether a given conclusion is robust to adding or removing higher-order effects. From a human perspective, the most interesting and insightful description arises from the simplest, most reduced, model that can describe the observations. In contrast, applications of MMNLFO may ultimately require quantitatively accurate modeling, or at least appreciation of all the details of the physics.

For the most part, these principles have been consistently successful. Nonetheless, the work is not perfect by any means. Mainly for posterity, two (ultimately relatively minor) mistakes are discussed in the next chapter. The first mistake, examined in section 2.2.3, significantly diverges from the intended summary-level description of the rest of this thesis to provide a new theoretical analyses of MM solitons that disproves our early hypothesis about the role of modal dispersion in determining the energy of MM solitons. The second is the failure to account for geometric parametric instabilities in the analysis of spatiotemporal dispersive radiation from oscillating MM solitons, discussed in Section 2.4.2. Neither mistake ultimately changes the conclusions of the original works, but in my opinion the added/updated insight is well-worth including here. 


\section{CHAPTER 2}

\section{SPATIOTEMPORAL DYNAMICS OF MULTIMODE OPTICAL SOLITONS}

In this chapter, we will examine nonlinear waves called multimode solitons which form in anomalous dispersion $\left(\beta_{2}<0\right)$ multimode fibers. MM solitons are robust nonlinear attractors, which tend to try to preserve themselves in face of higher-order perturbations or inter-soliton interactions. This robustness and nonlinear attraction makes them a conceptual framework to understand much more complex multimode dynamics.

My $\mathrm{PhD}$. research began with a handful of projects performed initially in collaboration with Will Renninger, who proposed them, and supervised me through their first four months. These projects were not quite entirely failures, and have not quite entirely been forgotten, as the last chapter will reveal. For one, they naturally led to the work described in this chapter, the first publishable work of my thesis.

Multimode solitons were first predicted by Hasegawa [28] (and virtually simultaneously by Crosignani[29]), albeit in models that neglected all terms besides the self- and cross-phase modulation terms appearing in the MMNLSE. Later, Yu et al. predicted spatiotemporal solitons in GRIN MMFs, using a 3+1 D equation similar to the 3+1 D GNLSE[30], a result which was later also obtained by Raghavan and Agrawal [31], and has since been advanced further by Shtyrina et al.[32]. Later in this chapter we will revisit these two classes of soliton predictions; they are not necessarily the same nonlinear waves.

Will Renninger first attempted to observe multimode solitons by launching pulses through a single-mode fiber spliced directly to a GRIN MMF [33]. While 
numerical simulations showed the essential behavior of MM solitons, the solitons were comprised of almost exclusively the fundamental mode of the waveguide, with higher-order modes merely being 'trapped' by the soliton in this lowest-order mode. Hence, the soliton acted precisely as one would expect a typical soliton of the 1+1 D NLSE, or in other words a single-mode soliton.

Later, I significantly extended this simply by launching light into the GRIN fiber through free space coupling with a lens, using translation of the fiber to excite different combinations of modes. A higher power laser allowed a variety of different MMS to be observed, as well as new spatiotemporal dynamics discussed in the next section. This study was our first foray into the complexity of multimode nonlinear fiber optics, and we were faced with a number of experimental challenges, as well as an overwhelming number of physical questions. As a consequence, with this study we asked quite conservative questions, namely:

1. Are MM solitons forming and propagating inside the fiber?

2. Do the dynamics of the soliton-like pulses agree with what simulations predict?

3. What are the dynamics of MM solitons? What happens when they interact or are perturbed?

Ultimately, and particularly with subsequent and ongoing studies with Zimu Zhu, we have convincingly answered the first two questions in the affirmative, albeit while raising plenty more new questions. For the last question, we do not yet have all the answers, but at least have some. 


\subsection{Linear propagation of pulses in multimode fibers}
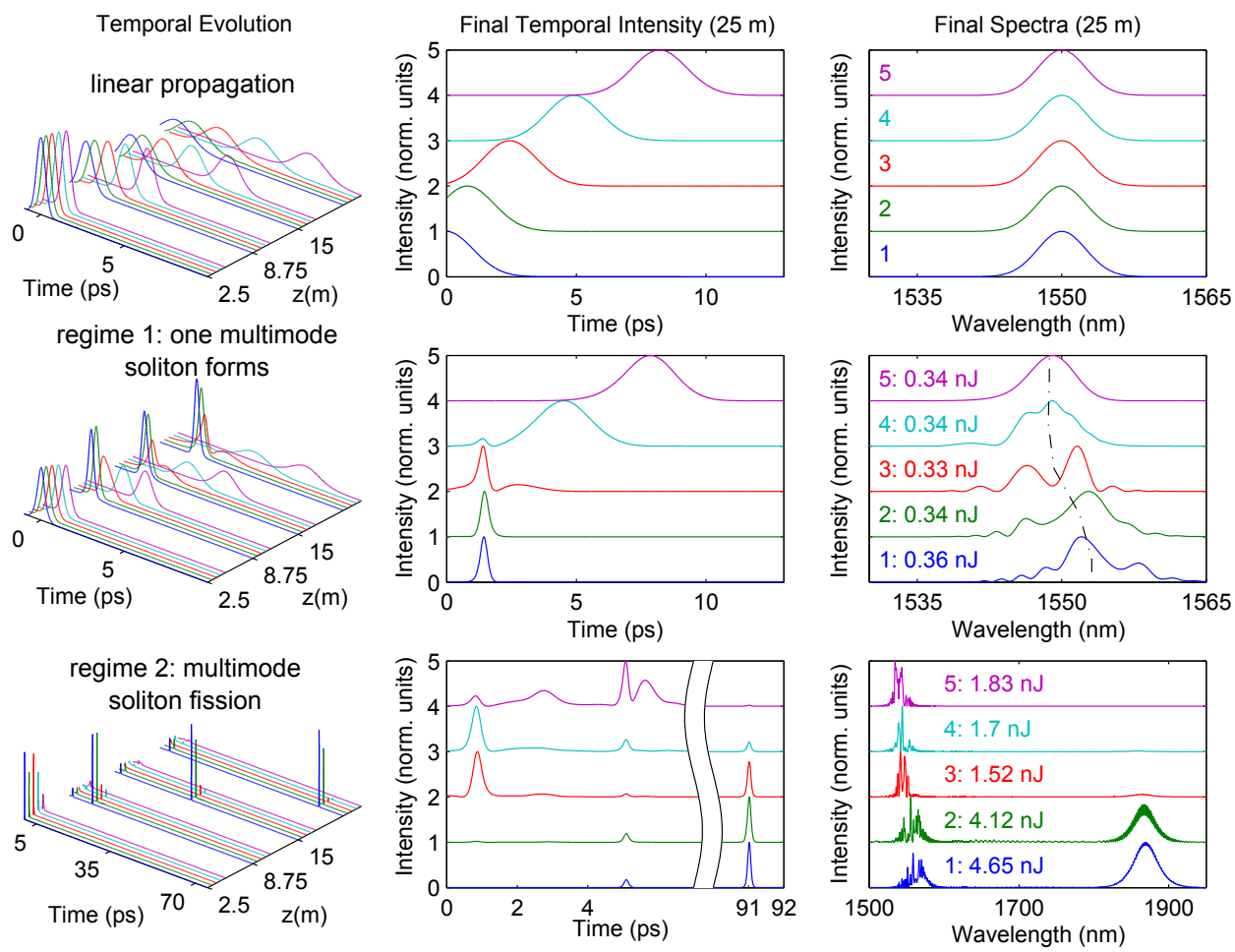

Figure 2.1: Propagation in anomalous dispersion GRIN MMF. The rows show three regimes of behavior for increasingly intense pulses launched into the fiber. The top shows the linear propagation. The middle shows the case where $1 \mathrm{MM}$ soliton forms from the launched pulse. The bottom row shows the spatiotemporal soliton fission regime, where numerous MM solitons are formed after an initial sequence of spatiotemporal compression.

The MMNLSE shows pretty clearly what linear pulse propagation in multimode fiber looks like. The linear part reads:

$$
\partial_{z} A_{p}(z, t)=i \delta \beta_{0}^{(p)} A_{p}-\delta \beta_{1}^{(p)} \partial_{t} A_{p}+\sum_{m=2}^{N_{d}} i^{m+1} \frac{\beta_{m}^{(p)}}{m !} \partial_{t}^{m} A_{p}
$$

Term by term, we can see that each mode develops propagation phase at a different rate, determined by $\delta \beta_{0}^{(p)}$. The second term simply corresponds to the different average velocities of pulses in different modes. This is called 'modal 
dispersion': at a given frequency, the group velocity of a pulse is different in different spatial modes. Last, the rest of the terms are chromatic dispersion terms which describe how different frequency components of a pulse in each given mode travel. In general, the chromatic dispersions in different modes can be radically different, but within this thesis we will not encounter situations where they significantly differ from one another.

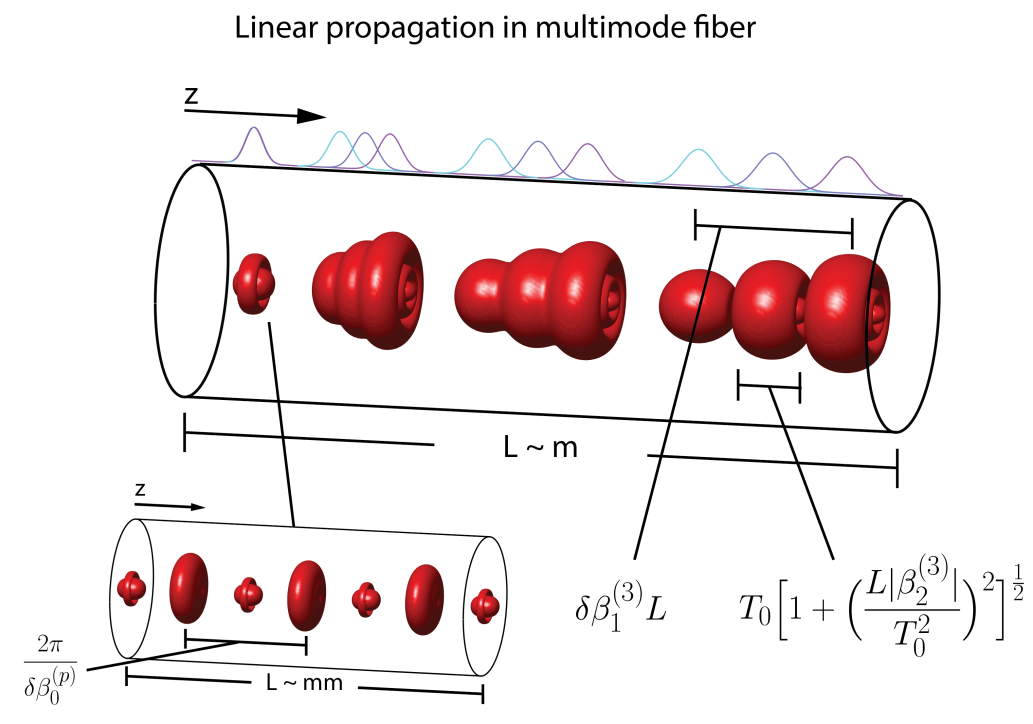

Figure 2.2: Linear MM propagation in spacetime. The mode-decomposed field at the output of the multimode cavity, in the (a) time and (b) frequency domains. Each radially symmetric mode has a different color, although only the three lowest-order radially symmetric modes have enough energy to be visible.

The top row of Figure 2.1 shows these terms in action. Starting from an initial condition where a pulse of equal duration and energy is launched into each of 5 different modes, over $25 \mathrm{~m}$ of fiber, the pulse in each mode broadens due to the last term(s), and moves away from the pulses in the other modes due to its unique modal velocity given in the second term. Not evident here is the first term, which corresponds to a very rapid (over roughly $1 \mathrm{~mm}$ ) oscillation of the beam when the pulses are overlapped. Figure 2.2 illustrates what this looks like 
the space-time domain, with the inset showing the fast oscillations due to the beating due to the first term.

\subsection{Multimode solitons}

\subsubsection{Solitons in $1+1 \mathrm{D}$}

If we just consider the reference mode in the MMNLSE, and we neglect higherorder chromatic dispersion, we have the NLSE for a single-mode fiber,

$$
\partial_{z} A(z, t)=-i \frac{\beta_{2}}{2} \partial_{t}^{2} A+i|A|^{2} A
$$

This equation also has modes, in some sense. That is, we can find solutions to the equation above for which $\partial_{z} A(z, t)=\hat{H} A(z, t)=\frac{i}{2 L_{d}} A(z, t)$, where $L_{d}=\tau^{2} /\left|\beta_{2}\right|$. These magnificent solutions are the fundamental solitons:

$$
A(z, t)=\sqrt{P_{o}} \operatorname{sech}(t / \tau) e^{i z / 2 L_{d}}
$$

Here, the sum of the phase introduced by the (anomalous: $\beta_{2}<0$ ) groupvelocity dispersion and the nonlinear self-phase modulation is a constant, so the soliton $A(z, t)$ propagates without changing its shape. The condition for this

is that $P_{o}=\frac{\left|\beta_{2}\right| A_{\text {eff }}}{n_{2} k_{o} \tau^{2}}$. Since experimentally we do not have easy direct access to the peak power, $P_{o}$, we usually have to be content with a slightly-less direct parameter, the pulse energy. If one integrates the power $|A(z, t)|^{2}$, the energy in the soliton can be shown to be $E_{p}=2 P_{o} \tau$, or: 


$$
E_{p} \tau=2\left|\beta_{2}\right| A_{e f f} / n_{2} k_{o}
$$

This is called the soliton energy-area theorem.

\subsubsection{Multimode solitons}

Multimode solitons, at least in the regime described in this thesis, are not strictly solitons. That is, the MMNLSE is not an equation for which we can find eigensolutions for. Nonetheless, in many respects multimode solitons act in the way we expect solitons to behave. The vast majority of waves that appear in the study of solitons, more carefully termed solitary waves, are like this[34].

How does a MM soliton work? When nonlinearity is included in the MMNLSE, the self-phase modulation causes each pulse to resist chromatic dispersion. The cross-phase modulations between pulses in different modes cause pulses in different modes to naturally shift their center frequencies in such a way that the intermodal dispersion is counteracted by the group velocity dispersion. For example, in the example shown in the second row of Fig. 2.1, the fundamental mode has the smallest value of $\beta_{1}$. Since furthermore the dispersion is anomalous, it redshifts (shifts to lower frequencies) so as to increase it's effective $\beta_{1}\left(\delta_{\omega}\right)=\beta_{1}(0)-\left|\beta_{2}\right| \delta_{\omega}$. A view of the soliton evolution is depicted in Fig. 2.3 


\section{Multimode solitons in multimode fiber}

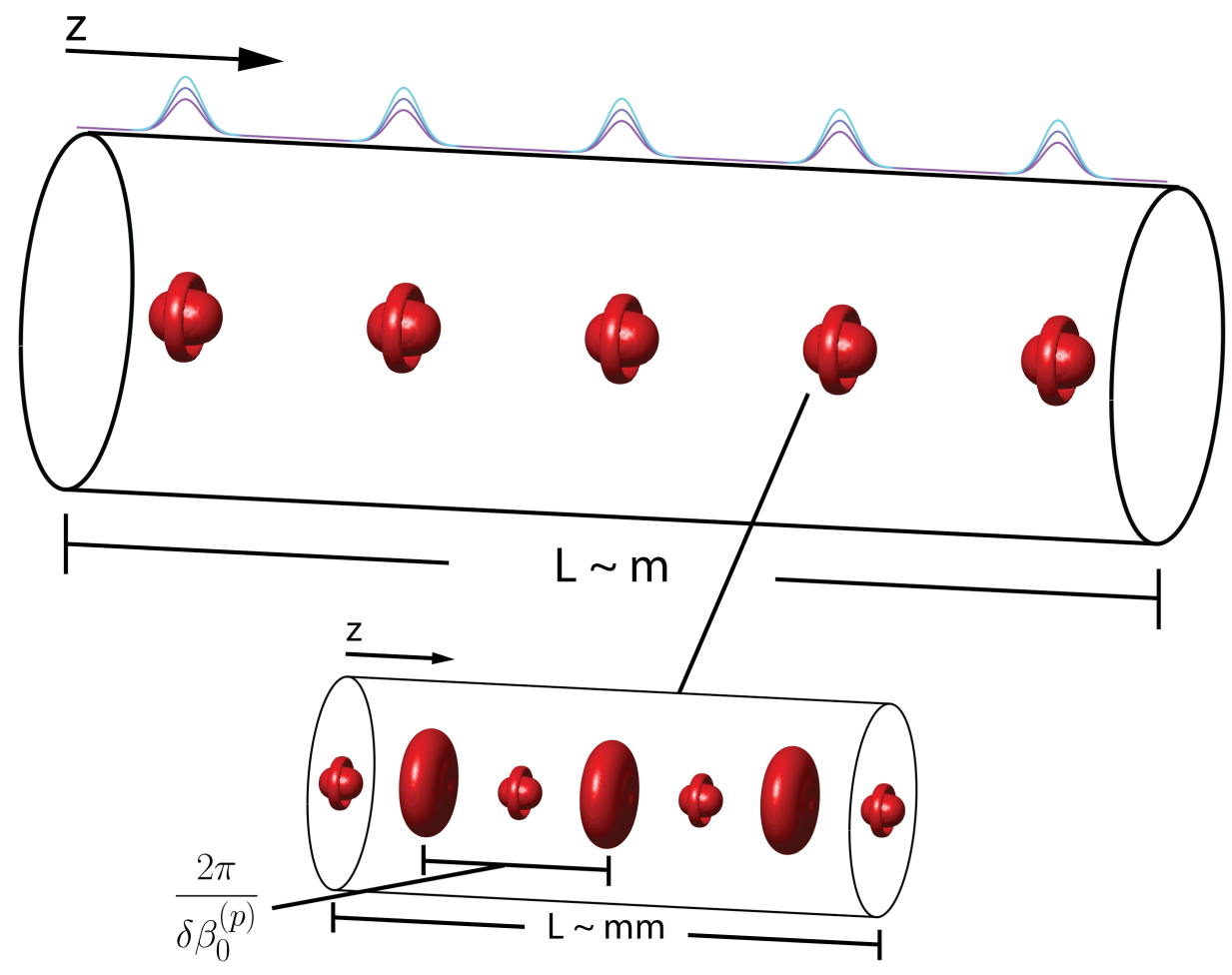

Figure 2.3: Multimode solitons in spacetime. The mode-decomposed field at the output of the multimode cavity, in the (a) time and (b) frequency domains. Each radially symmetric mode has a different color, although only the three lowestorder radially symmetric modes have enough energy to be visible.

\subsubsection{MM soliton energy}

One natural question to ask is 'Is there an analogue of Eqn. 2.4, an energyarea theorem, for MM solitons?' Here I consider this question with a new analytic model for the MM soliton, which I examine in some detail. This model highlights deficiencies in our earlier understanding and assumptions about MM solitons, so in this section and the next I correct these works [33, 27, 35].

Let us start by considering a 2-mode soliton where, for some reason, the four-wave mixing terms of the MMNLSE are zero, and the two modes have 
identical values of $\beta_{0}, \beta_{2}$ and $A_{\text {eff }}$, but different values of $\beta_{1}$, differing by $\delta \beta_{1}$. We will examine some of these deficiencies subsequently. Despite obviously not representing a true MM soliton, a solitary wave of the full MMNLSE, we can gain some insight from this simplified special case. We will also extract some insight from its failures to be a fully accurate description too.

In this situation, in the additionally special case that the amplitude in each mode is identical, a solitary wave solution can be found[13, 36]:

$$
A_{1,2}(z, t)=\sqrt{P_{o}} \operatorname{sech}(\sqrt{1+B} t / \tau) e^{i z(1+B) / 2 L_{d}+i \delta \beta_{1} / 8\left|\beta_{2}\right| z \pm i \delta \beta_{1} t / 2 \beta_{2}}
$$

where the modes are assumed to have different values of $\beta_{1}$, differing by $\delta \beta_{1}$, but the same $\beta_{0}, \beta_{2}$ and $A_{e f f}$, and where $B$ is the coefficient of the cross-phase modulation. If we examine the phase, we see that the main soliton phase, the soliton propagation constant or wavevector, is modified by $B$. The second phase term arises from the shift of the propagation constant due to the frequency shift. Since the component arising from $\beta_{1}$ is canceled by the frequency shift for each mode, this is just $\Delta \beta_{0}=\frac{\beta_{2}}{2}\left(\delta_{\omega}\right)^{2}$. The third term is due to the frequency shift, which is in this case symmetric as the pulse in each mode shifts by an equal amount in opposite directions to reach equal group velocity.

If we integrate the power of the soliton in each mode and take the sum to get the total energy, we find $E_{p}=2 P_{o} \tau \times 2 / \sqrt{1+B}$. From this, we see that if $B=0$, we have the energy of two 'free' solitons. For $B$ between 0 and 3 , the total energy is larger than a single soliton, but smaller than 'free' solitons in each of the modes. For $B>3$, the energy of the MM soliton may actually become smaller than one uncoupled $1+1 \mathrm{D}$ soliton. This can all be thought of as a soliton molecule: just 
as atoms may prefer to form a molecule if it lowers their energy, a MM soliton forms in a MM fiber because it represents a reduction of the soliton-associated energy. If we let $T_{m}$ be the actual duration of the MM soliton, $T_{m}=\tau / \sqrt{1+B}$, we have $E_{p}=4 P_{o} T_{m}$. Hence, from one point of view the energy area theorem is modified as:

$$
E_{p} T_{m}=2(1+B) \times 2\left|\beta_{2}\right| A_{e f f} / n_{2} k_{o}
$$

We can attempt to generalize the result for the two-mode soliton to an $\mathrm{N}$ mode soliton, assuming all $\mathrm{N}$ modes have equal contributions:

$$
E_{\text {tot }}=\sum_{m=1}^{M} \sum_{l=1}^{M}\left(B_{l m}\right) 2\left|\beta_{2}\right| A_{e f f} / n_{2} k_{o} T_{m}
$$

Here, $E_{i}, T_{i}$, are the energies, durations, peak powers, of the pulses in each mode, while $A_{\text {eff }}$ is the effective area of the fundamental, and the $B_{i j}$ are the phase modulation coefficient between the modes, $S_{l l m m}^{K}$ and $S_{m l m l}^{K}$. Typical values of $B_{i j}$ for the MMNLSE in GRIN fiber are $<1$, with most being $<<1$. For a 3 mode soliton comprising the lowest-order modes of a GRIN fiber, and for which all modes have the same polarization and the pulse in all modes is the same duration $T_{m}=T$, the above equation would be:

$$
E_{\text {tot }}=6.594 \times 2\left|\beta_{2}\right| A_{e f f} / n_{2} k_{o} T
$$

A plausible way to generalize this result to a MM soliton comprising unequally-distributed modes, retaining the assumption that all modes share the same duration, $T$, is by scaling the phase-modulation coefficients by the energy 
in the mode $l$ relative to the energy in the first mode. Figure 2.4 shows the factor multiplying the fundamental mode area theorem for different distributions of energy in the modes.

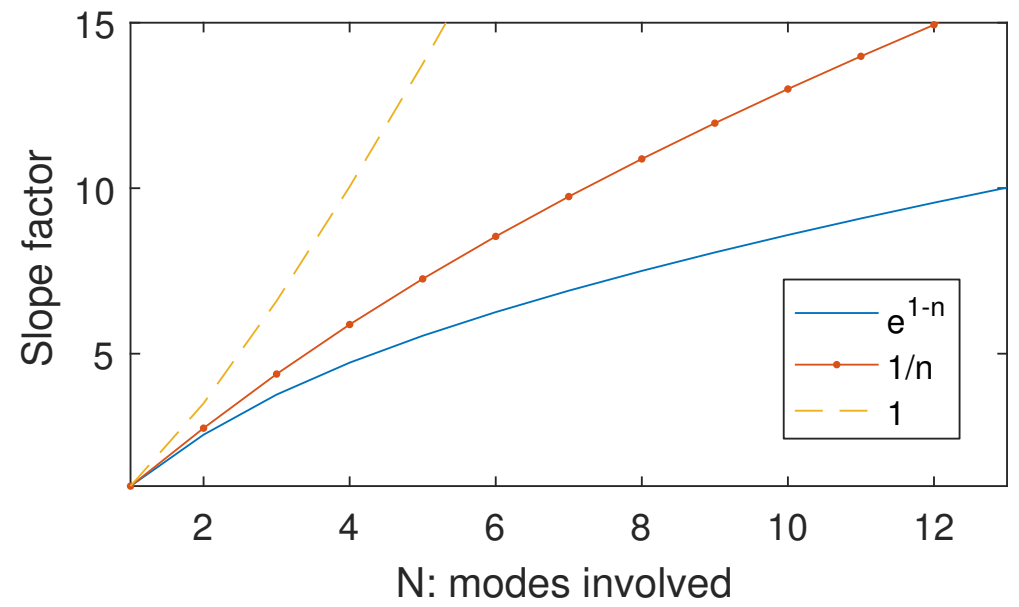

Figure 2.4: Theoretical guess at the factor modifying the energy-area theorem for multimode solitons compared to a soliton in the fundamental mode. The different curves show the factor for different distributions of energy within the MM soliton.

With these results laid out, let us examine why they are probably only reasonable guesses. That is, let us revisit the deficiencies in this 'N-mode soliton' model as a description of the real MMNLSE solitary waves like the those depicted in Figs.2.3 and 2.1 First, the energy in each mode is not necessarily equal, nor is the pulse duration in each mode necessarily the same. Second, of course the modes will have different $A_{e f f}$, and the cross-phase modulation between each pair of modes will have a different value of $B$. Third, the modes may also interact through phase-sensitive four-wave mixing terms, through which energy may be redistributed in the MM soliton's constituent modes. Finally, the modes will also have their own propagation constants, described by the first term in the MMNLSE. Of these, perhaps the most important is the possibility of four-wave mixing, since through energy exchange between the modes the 
MM soliton exhibits a character which is much closer to a single soliton entity than a 'composite soliton molecule'. Another feature of importance will be the different propagation constants, which underly instabilities of MM solitons considered later in this chapter.

Given all these issues, we should be conservative in taking intuition from Eqn. 2.7 and the preceding discussion. That being said, combined with the deficiencies, we can make several hypotheses (which, in our limited studies so far appear to be true).

1. A MM soliton exists (is a stable nonlinear attractor) because it minimizes energy relative to $M 1+1 \mathrm{D}$ solitons propagating independently in the modes.

2. A MM soliton will generally have more energy than a SM soliton of the same total area and duration, since pulses in different modes exert weaker influence on one another than pulses in the same mode.

3. At least to first order, the energy of a MM soliton does not depend on the modal dispersion. However, to excite a MM soliton from an initiallytransform limited pulse intuitively requires more power and/or more initial bandwidth if the modal dispersion is stronger. Nonetheless, even with very strong modal dispersion a MM soliton may be excited, provided the exciting pulse is close to the MM soliton attractor (which would require pulses in different modes having very different center frequencies).

One way to understand this behavior is to examine the intensity of the MM soliton. When two solitons propagate independently in different modes, the sum of their intensities is incoherent, $I_{1}+I_{2}$. If pulses instead form a composite 
soliton, in which they overlap in polarization, phase, time and space precisely, the intensity may be increased by up to 4 -fold. Even if the solitons lock in phase and time, the less solitons overlap in space (or polarization) the less will be the increase in intensity resulting from their overlap. There will nonetheless be an increase in the intensity provided the modes have some overlap. This increase in intensity is the reason why the MM soliton is a lower-energy configuration than multiple single-mode solitons. On the other hand, combining solitons in multiple modes does not increase the intensity as much as if one were to simply take all that energy and place it in a single mode soliton (in which case all the added intensities sum coherently). This inefficiency is why the MM soliton will generally have higher energy than a single-mode soliton with comparable intensity.

Before continuing to the next section, it is worth emphasizing that most, but not all of this analysis is consistent with our initial work on MM solitons. One particular place where it disagrees however, is in the importance of modal dispersion on the soliton energy. In Ref. [27], we argued that modal dispersion meant that MM solitons should have a energy-duration relationship that was similar to single-mode solitons, as in the area-theorems discussed, but that MM solitons should exhibit a larger slope of $E$ vs $1 / \tau$ compared to solitons in the fundamental mode, or even single-mode solitons with the same equivalent area. While evidently this is true, it is true for different reasons than we claimed. MM solitons generally exhibit a larger energy because of the inefficient nonlinear coupling of the different modes within them relative to nonlinear coupling within a given mode. We probably made this error by wrongly assuming that MM solitons are a sort of 'waveguide-aided' spatiotemporal soliton; this is not true as the next section explains. Second and most primarily, this error is sup- 
ported by simulations and experiments in that the threshold to excite a given MM soliton from an initially-narrowband single pulse certainly does depend on the modal dispersion.

Finally, despite its clear deficiencies, the theoretical model described here seems to be at least roughly consistent with those earlier experiments. Both the simulations and experiments of MM solitons shown in Fig. 3 of Ref. [27] agree very well in shape and magnitude with the prediction of a exponentiallydistributed MM soliton from Figure 2.4, which is a reasonable description for the Gaussian-shaped solitons considered there.

\subsubsection{Spatiotemporal solitons in MMF versus MM solitons}

As mentioned, a secondary source of our initial mis-attribution of the role of modal dispersion in MM solitons stems from a presumed equivalence between two categories of predicted solitons. While in detail the situation is a little more nuanced, we can nonetheless distinguish these solitons as fundamentally different kinds of solitary waves.

The MM solitons considered in this chapter most closely resemble those predicted by Hasegawa and Crosignan[28, 29]. In the limit of infinitely-many modes, the MM solitons considered throughout this chapter are something of a soliton-molecule O-wave[37]. Wavelength shifts of different modal components counteract modal dispersion, and ultimately, the 'modal dispersion' of diffraction.

In contrast, the 'spatiotemporal solitons' predicted elsewhere[30, 31, 32] 
should be differentiated. Here, the physical picture is a soliton that, by adding an additional spatial inhomogeneous phase to the GRIN waveguide, forms a single-mode waveguide (or at least finds itself in the fundamental mode of said waveguide). In the low-energy regime, such a soliton must be in the fundamental mode of the linear waveguide. With increasing energy, the average spatial size of this soliton must shrink relative to the linear waveguide's fundamental mode, and in the limit of infinitely many modes the soliton's spatial size is infinitely smaller than the fundamental plane wave. It is then a full 3+1 D spatiotemporal soliton, in which nonlinearity directly counteracts diffraction and dispersion simultaneously (note however the solutions do not predict stability in this regime; STS in 3+1 D are unstable[38]).

It is possible that future work, discussed in the final chapter, will be able to observe the second category of soliton, either by directly exciting it using spatial light modulators, or in multi-pass 'waveguides'.

\subsection{Spatiotemporal fission and Raman scattering of multimode solitons}

Before examining experimental observations of $\mathrm{MM}$ solitons, it is beneficial to first examine what happens when we launch a pulse whose energy exceeds that for which a single MM soliton forms.

Fig. 2.1 is a pretty good representation of what happens in typical experiments and simulations when one launches a MM pulse into a MM GRIN fiber

and increases the energy $[39,27,40,35]$. At low power, the pulse broadens due 
to linear effects. At higher power, a single MM soliton forms, with possibly some dispersive radiation escaping in certain modes. This 'single MM soliton' regime lasts until some threshold power for multiple MM solitons, or soliton fission, which in general depends on the initial modal composition and the pulse parameters. In this fission regime, there is too much energy in the initial pulse to be stored easily in just one MM soliton, so it ultimately blows up into several (plus some dispersive radiation). At and beyond the threshold power, the launched pulse compresses strongly in time, as well as typically in space, with energy flowing from higher-order to lower-order modes. At some point along the fiber, this compression is arrested and the pulse fissions in spacetime: it breaks up into many different pulses, as well as dispersive radiation, that then move apart in space as well as time.

Fig. 2.1's bottom panel shows a typical outcome. An intense MM soliton emerges from this 'spatiotemporal fission' in relatively low-order modes, and is redshifted and decelerated, moving rapidly away from the rest of the field. This red-shifting results from coherent Raman gain of the red side of the soliton's spectrum by the blue-side. While this effect is well-known for solitons in SMF[41, 42, 43], it is noteworthy that MM solitons also exhibit soliton selffrequency shift, remaining MM solitons as they shift. Intuitively, however, depending on which modes predominantly occupy the red side of the MM soliton spectrum, the MM soliton's modal distribution may change.

The rest of the 'fissioned' pulse also forms into different MM solitons. Besides the Raman-shifted MM soliton, at least two, and probably 4 MM solitons can be observed in the middle lowest plot of Fig. 2.1. This spatiotemporal fission into multiple MM solitons shows clearly why MM solitons are useful in under- 
standing complex nonlinear optical pulse propagation in MMFs, and illustrates how they are the stable configurations of high-intensity light in anomalousdispersion MMFs (even more than solitons in the individual modes).

To describe pulse propagation in the third regime, we need to consider higher-order effects beyond the MMNLSE. These higher-order affects are the cause of the arrested spatiotemporal compression of the field, prompting a break-up into stable MM solitons. The so-called generalized MMNLSE, originally derived by Poletti and Horak [22, 23] includes the Raman effect, which like the Kerr nonlinearity couples all the modes together, and self-steepening.

$$
\begin{aligned}
\partial_{z} A_{p}(z, t)= & i \delta \beta_{0}^{(p)} A_{p}-\delta \beta_{1}^{(p)} \partial_{t} A_{p}+\sum_{m=2}^{N_{d}} i^{m+1} \frac{\beta_{m}^{(p)}}{m !} \partial_{t}^{m} A_{p} \\
& +i \frac{n_{2} \omega_{o}}{c}\left(1+\frac{i}{\omega_{o}} \partial_{t}\right) \sum_{l, m, n}^{N}\left[\left(1-f_{R}\right) S_{p l m n}^{K} A_{l} A_{m} A_{n}^{*}\right. \\
& \left.+f_{R} S_{p l m n}^{R} A_{l} \int_{-\infty}^{t} d \tau h_{R}(\tau) A_{m}(z, t-\tau) A_{n}^{*}(z, t-\tau)\right]
\end{aligned}
$$

Here, the Raman contribution to the third-order optical nonlinearity is represented by the 'Raman fraction', which for fused silica is about 0.18 , and the phenomenological Raman impulse response, $h_{R}[13]$. The Fourier transform of $h_{R}$ is related to Raman gain spectrum, which describes the tendency for the Raman effect to, with phase-matching and energy conservation ensured by a phonon within the glass, amplify a red-shifted wave. The Raman gain spectrum is broad, with a maximum value at $-13.5 \mathrm{THz}$ from the initial field (i.e., the center frequency of $\left.A_{p}(z, t)\right)$. In a soliton (or MM soliton), all its frequencies overlap precisely in time (it is a transform-limited pulse), and thus the blue side (high frequency) side of the soliton spectrum may amplify the red-side of the 
spectrum, creating the aforementioned soliton self-frequency shift.

If we keep our ongoing assumption that only linearly-polarized fields in a single polarization are relevant, the tensors are simple:

$$
\begin{aligned}
S_{p l m n}^{K} & =S_{p l m n}^{R} \\
& =\frac{\int \mathrm{d} x \mathrm{~d} y\left[F_{p} F_{l} F_{m} F_{n}\right]}{\left[\int \mathrm{d} x \mathrm{~d} y F_{p}^{2} \int \mathrm{d} x \mathrm{~d} y F_{l}^{2} \int \mathrm{d} x \mathrm{~d} y F_{m}^{2} \int \mathrm{d} x \mathrm{~d} y F_{n}^{2}\right]^{1 / 2}}
\end{aligned}
$$

Refs. [27] and [40] contain more details about the fission process in highlymultimode GRIN fiber. However, one thing that spatiotemporal fission and the Raman self-frequency shift allow is a clean observation of isolated MM solitons. This is because exciting a MM soliton from a pulse is not usually a fully efficient process, as the middle panel of Figure 2.1 illustrates. The formed MM soliton propagates at the same wavelength as any dispersing radiation, and consequently somewhat elaborate schemes need to be developed to account for it [27]. In contrast, the Raman-shifted MM soliton can be isolated by a long-pass spectral filter. This is what I discuss in the next section.

\subsubsection{Observations of multimode solitons}

As mentioned, Refs. [39] and [27] observed MM solitons in GRIN fibers with many modes, by directly measuring MM solitons in the 'single soliton regime'. In both cases, the measurements exhibit good agreement between simulations, experiments, and theory. Especially if you have read section 2.2.3, I encourage you to re-examine these references. 

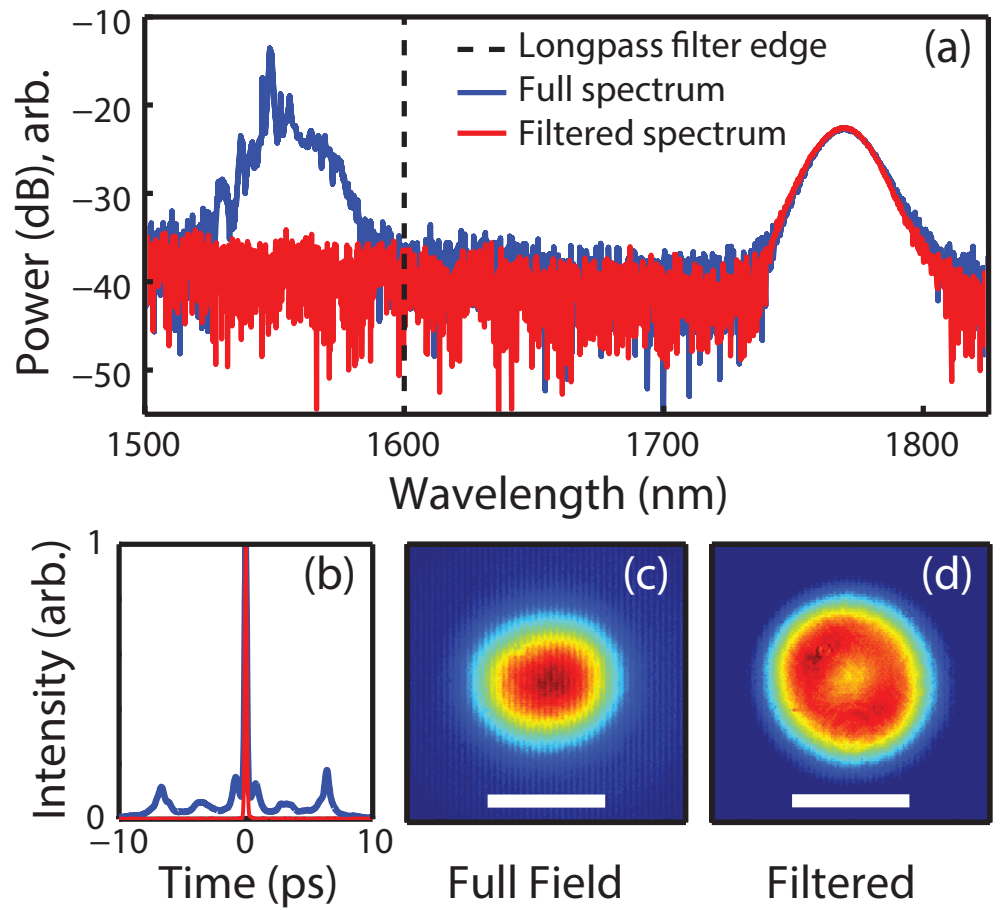

Figure 2.5: Direct observation of a few-mode Raman-shifted MM soliton. (a) shows the spectrum of the entire field exiting the few-mode GRIN fiber, along with the isolated soliton spectrum after spectral long-pass filering. (b) shows the intensity autocorrelations before (blue) and after (red) this spectral filtering, illustrating the isolation of the single MM soliton in the time domain. (c) and (d) show the near-field beam profiles of the full-field and the field after spectral filtering (scale bar is the fundamental mode diameter). The intensity profile in (d) can only be accounted for by all 3 spatial modes of the fiber, LP01, LP11a and LP11b, and thus is a MM soliton.

That being said, in the interest of providing the clearest and most compact evidence for MM solitons, I will rely on work performed with (and primarily performed by) Zimu Zhu [35], who as a new PhD student filtered out all the failures of his predecessors into a beautiful, simple (at least relatively speaking) demonstration of MM solitons. In this work, we eliminated much of the complexity of our earlier studies by considering a few-mode fiber (supporting just 6 modes, 3 spatial with 2 polarizations, instead of the 100s of previous works) and focused on the spectrally-isolated Raman-shifted MM soliton. Example data 


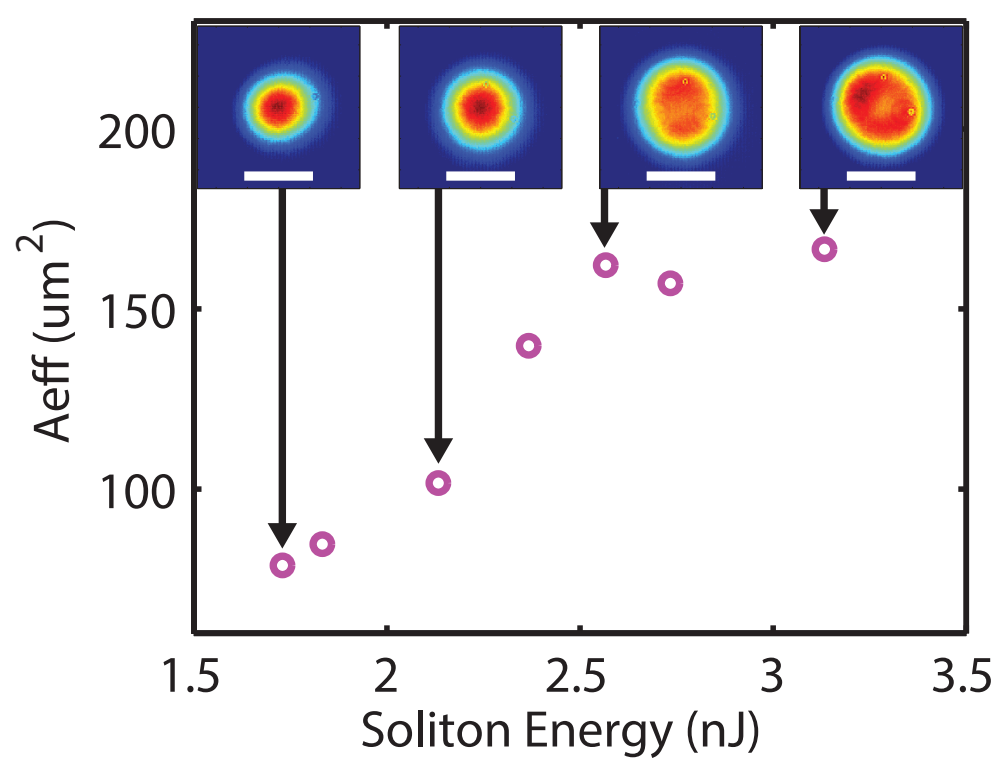

Figure 2.6: Changing $A_{\text {eff }}$ of the Raman-shifted soliton for increasing soliton energy. MM Raman solitons were spectrally isolated by a longpass filter. For increasing launched pulse energy, this isolated Raman soliton exhibits a similar pulse duration, but changing spatial shape. At low energy, the MM soliton comprises primarily the fundamental mode. At higher energy, the soliton's energy is roughly equally distributed among the 3 spatial modes of the fiber.

from this study is shown in Fig. 2.5.

This work also permitted clearer observations of the 'spatiotemporal adaptability' of MM solitons. As mentioned, due to the FWM terms (and Raman) in the GMMNLSE, MM solitons can exchange energy between their modal components. Fig. 2.6 shows how changing the energy of the MM soliton affects its spatial mode distribution, here described by the beam profile and the effective area, $A_{\text {eff }}=\left[\iint|E(x, y)|^{2} \mathrm{~d} x \mathrm{~d} y\right]^{2} /\left[\iint|E(x, y)|^{4} \mathrm{~d} x \mathrm{~d} y\right]$. This result is not of a single MM soliton in a changing environment, rather it shows how the spatiotemporal fission process changes with increasing initial pulse energy. However, it illustrates how MM solitons act as a kind of 'spatiotemporal solitons', in the sense that they have coupled spatial and temporal degrees of freedom. 


\subsection{Spatiotemporal oscillations of multimode solitons}

As was mentioned earlier, one deficiency of the analytic MM soliton suggested in section 2.2.3 is that it neglects the intermodal beating, $\delta \beta_{o}^{(p)}$ between the different modes of the soliton. These oscillations mean that the soliton, while unchanging in time, changes in space - it periodically oscillates. In GRIN fiber, this oscillation is particularly simple because modes are separated into groups with equally-spaced propagation constants, so all MM fields oscillate with a period equal to or at multiples of $P=1 / \Delta \beta=\sqrt{n_{o} \pi^{2} R^{2} / 2\left(n_{o}-n_{\text {cladding }}\right)}$ as depicted in Fig. 2.3. This is because parabolic GRIN fiber is just another simple-harmonic oscillator potential:

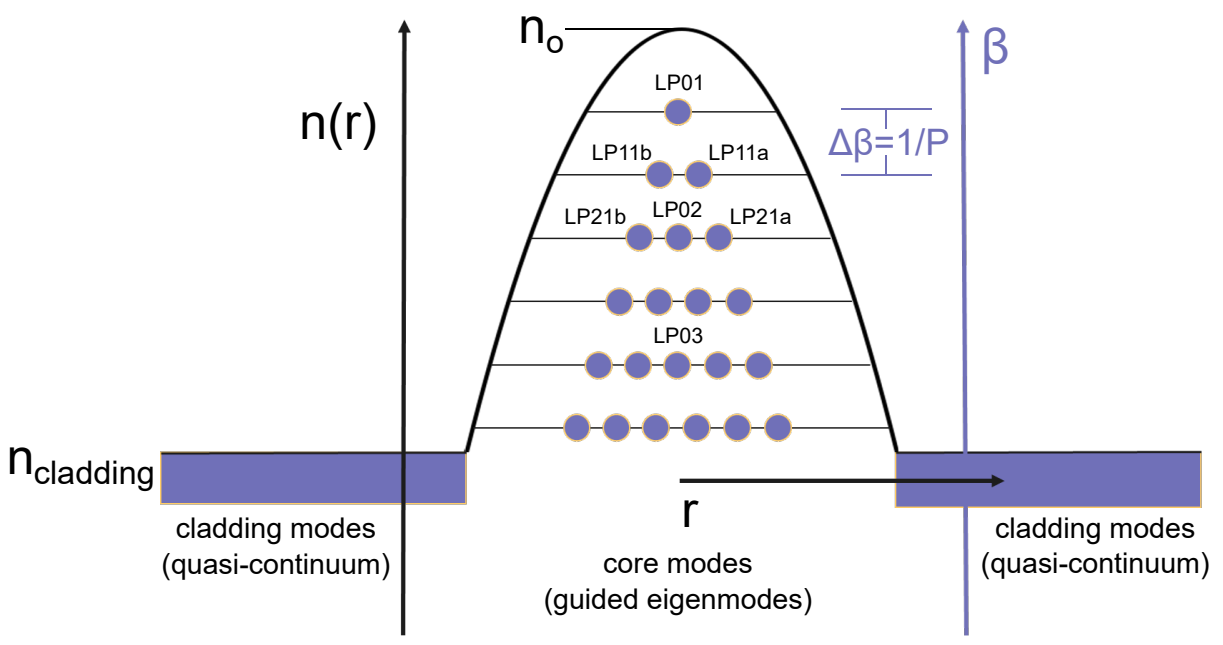

Figure 2.7: Mode structure of a parabolic potential. A parabolic optical potential, as most GRIN fibers are, has groups of quasi-degenerat modes separated whose propagation constants, $\beta$ are separated by equal amounts, equal to $\Delta \beta=1 / P$

These oscillations pose some big problems for MM solitons. Recalling earlier sections, a soliton's phase evolves with a wavevector $k_{\text {sol }}=\left|\beta_{2}\right| / 2 \tau^{2}$. One fantas- 
tic feature of solitons (and MM solitons, to the extent we can tell so far) is that, if the environment they propagate through changes, they tend to naturally reshape to preserve the soliton energy-area theorem. This is the reason they seem to elegantly remain solitons even as they are decelerated by the Raman effect.

\subsubsection{Oscillation instability of solitons (including MM soli-}

\section{tons)}

If the changes to the soliton environment occur over length scales much longer than the soliton's phase evolution period, $Z_{o}=2 \pi / k_{\text {sol }}=4 \pi \tau^{2} /\left|\beta_{2}\right|$, the soliton will tend to losslessly re-adjust. In contrast, if the changes occur over a similar length scale the soliton cannot adjust fast enough - it simply cannot change fast enough to become a perfect soliton in the new environment. In this case, at some instant the soliton will cease to be a soliton, but can instead be thought of as a collection of light that includes a perfect soliton. In ensuing propagation, the light in the collection that is not part of the new perfect soliton within it will be shed from the soliton, radiated away as so-called dispersive radiation (dispersive because it is just linearly propagating light that is not part of a non-dispersive soliton).

If the changes to the soliton's environment are periodic, with some period $Z_{p}$, then at each instant the difference between the old, perfect soliton, and the new, slightly imperfect soliton, will be a field with some spectral structure. Since the perfect soliton field is changing periodically with the oscillations of the environment, it is clear that the 'difference' field will also be periodically changing. Light with this characteristic has a phase which is related to the difference between the soliton period and the period of the changing environment. In terms 
of the wavevectors,

$$
k_{d i s}=k_{s o l}-2 m \pi / Z_{p}
$$

Here, $k_{d i s}$ is the wavevector of the dispersive radiation, the 'difference field'. This equation is a phase-matching condition, which implies that linear dispersive radiation with the correct phase evolution is phase-matched to the difference between the soliton and the changing conditions, which impart a virtual momentum $2 \pi / Z_{p}$. This phase-matching is valid for any integer multiple of the the latter, hence the $m$ which is an integer.

The wavevector of linearly propagating fields is, relative to the center frequency of the soliton, a quadratic function of the frequency difference $\Omega, k_{\text {linear }}=$ $k_{d i s}=\beta_{2} \Omega^{2} / 2$. Thus, one can solve the phase-matching condition for the frequency of the dispersive radiation that satisfies it:

$$
\Omega_{m}=\frac{1}{\tau} \sqrt{\frac{2 \pi m}{k_{s o l} Z_{p}}-1}
$$

It is a very reasonable hypothesis that this instability applies equally to a MM soliton. While it is intriguing that a MM soliton may redistribute energy amongst its modes in addition to changing its shape in time, it is still limited by a maximum rate of change. Provided we use $\tau$ as the real pulse duration of the MM soliton, its wavevector is the same as that of a single-mode soliton, and we can apply this analysis more or less directly.

The MM soliton's own spatial oscillations present an instability for itself. In this view, the 'ideal' MM soliton is one like Eqn. 2.5, where the soliton does 
not actively 'perturb itself'. The MM soliton of reality is, unfortunately, much more self-destructive. A MM soliton is really only stable when its own internal spatial oscillations, due to mode beating, are much faster or much slower than its own phase evolution, $2 \pi / k_{\text {sol }}=4 \pi \tau^{2}\left|\beta_{2}\right|$. As its duration decreases and this period approaches the beating period (for a GRIN fiber, $P$ or multiples of it), the MM soliton will radiate energy into these specific frequencies until it's duration is sufficiently long enough to 'not see' its own oscillations.

\subsubsection{Cataloguing the instabilities of MM solitons and waves in GRIN fiber}

Before entering the next section, where some of the most exciting results of my $\mathrm{PhD}$ are discussed (including experimental measurements of the dispersive waves just described), let us examine the second of the errors alluded to earlier. Ultimately it is not clear this qualifies as an 'error' as much as an error we could easily have made, but I list it here as another detailed point for posterity.

If one examines Fig. 2.7, it is clear that if one excites only the radiallysymmetric modes LPON, that the effective period of oscillation, $P$, is actually twice as short, since an LPON mode is only found every second mode group. Thus, if one considers purely these modes, the period of oscillation $Z_{p}$ is not actually the nominal pitch of the fiber, but half as short.

Coincidentally, a complementary four-wave mixing instability exists in GRIN fibers (or any nonlinear dispersive medium with four-wave mixing and

periodic perturbations). This was later dubbed a 'parametric geometric insta- 
bility' by Katarzyna Krupa and colleagues in their paper [44] published just 7 months after our paper on space-time oscillation induced dispersive radiation [45]. However, at the time I knew this instability as one described by Matera et al. for single-mode fields[46]. While the geometric instability will be described later in the next chapter, the basic concept is similar to the soliton dispersive wave one. However, unlike dispersive radiation emission, the parametric instability is a four-wave mixing process applying to a single-frequency field, not necessarily a soliton.

The difference between these two manifests materially. Through the soliton's wavevector, the separation between sidebands due to dispersive radiation and GPI four-wave mixing, for a given period $P$, is different by a factor of $\sqrt{2}$. That is,

$$
\begin{aligned}
& \Omega_{m, O D W} \propto \sqrt{\frac{4 \pi m}{\left|\beta_{2}\right| Z_{p}}} \\
& \Omega_{m, G P I} \propto \sqrt{\frac{2 \pi m}{\left|\beta_{2}\right| Z_{p}}}
\end{aligned}
$$

As the next section will show (and Ref. [40] shows with numerous examples), it is possible to observe both processes with high-power pulses in the anomalous dispersion regime (as well as others related to spatiotemporal modulation instability, which will be described in the next chapter). In theory and simulation, these processes can overlap (for example, I am relatively sure the unusual exponential growth of some of the sidebands observed in Ref. [45] results from this). In Ref. [45], we derived the period directly from the space-time 
oscillation period of the solitons, which is a good way of being relatively sure of the correct origin of the radiation (that is, if the MM soliton is oscillating in space-time at some period, it is radiating dispersive waves whose frequencies are predictable using the formula for $\Omega_{m, O D W}$ ).

In experiments, however, it is easy to see that depending on which modes one excites, there may actually be no way to tell from the final beam profile and the spectrum which process is occurring. Thus, while it seems fairly likely that the experimental data presented in Ref. [45] shows the intended dispersive waves, rather than GPI sidebands, it is difficult to be sure since dispersive wave sidebands arising from a MM soliton in a given set of modes can precisely overlap with the GPI sidebands of a field in another set of modes. This is complicated further by the fact that the precise parameters of the experimental GRIN fibers used are not known, especially at 1550-nm. Thus, if I could rewrite Ref. [45], I would, in addition to including a clarifying note like this section in the supplementary information, would have included experimental data for the most widely-spaced sidebands observed experimentally. These sidebands are shown in Figure 2k and in Ref. [40], as well as in Figure 2.8c, where they are the second from the bottom, and Figure 2.8c. The beam profiles of these sidebands (and the pump field in Figure 2k in Ref. [40]) confirms these sidebands correspond to a fairly pure excitation of the LPON modes. Since these are the sidebands with the largest possible separation of any process excitable by a Gaussian beam launched into the fiber, they can be most unambiguously identified as dispersive waves from space-time oscillations of multimode solitons. 


\subsection{Complex nonlinear optics: controlling the bewildering range of nonlinear effects}

In mid-late 2014, we borrowed a high-power 1550-nm ultrafast fiber laser from Chris $\mathrm{Xu}$, and proceeded to thoughtfully launch as much power into short GRIN multimode fibers as possible. Of particular interest were the mysterious spectral 'peaks' we observed, which as described in the previous section included at least GPI and oscillation-induced dispersive waves, but probably also different intermodal, or spatiotemporal, modulation instabilities and the corresponding dispersive radiation processes. Coupled with the capacity to control the characteristics of the excited MM solitons by changing the modes initially excited, I soon realized that to a significant extent, I could generate about any spectrum I desired, or to a lesser extent any spatiotemporal field, just by tuning the initial modes excited and the power. Operationally, this meant that in the laboratory I adjusted the position of the exciting beam relative to the fiber using a 3-axis translation stage. A summary of the experiment is shown in Fig. 2.8.

This work, while severely lacking in the kind of factual conclusions that one often wishes for in a scientific publication, really emphasizes the exciting, bewildering and even overwhelming multitude of things that can be explored in MMNLFO. It is, if not a success of answers, a success of questions. In contrast to the role of science outside science itself, the questions are often more important.

This work also shows that, to a large extent, MM solitons can provide a conceptual framework for understanding more complex dynamics. However, as the previous sections have suggested, they fall short and even a PhD student deep into the details may struggle to conceptually unpack complex MMNLFO 
(a)
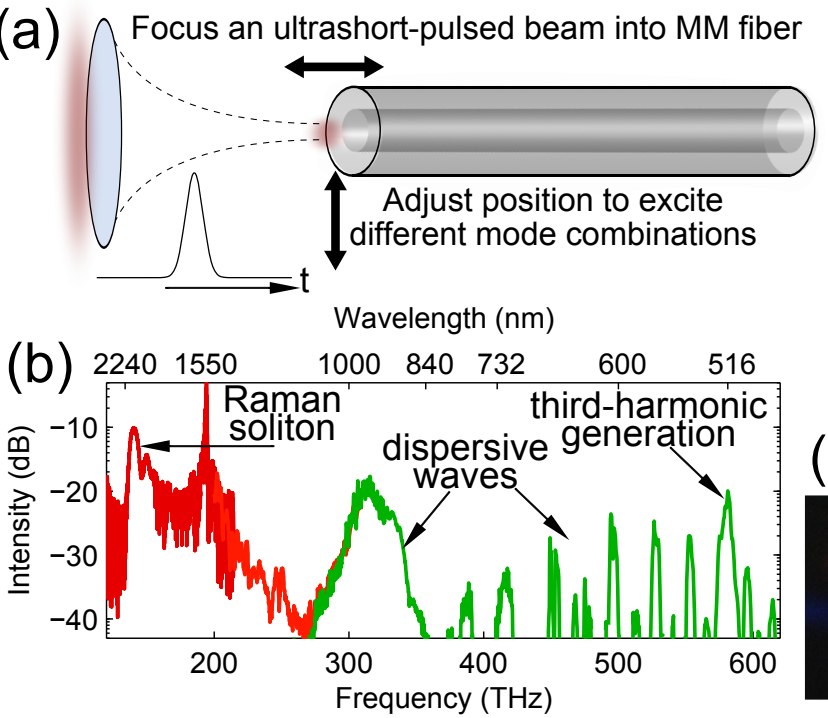

(C) Different initial conditions

Wavelength $(\mathrm{nm})$

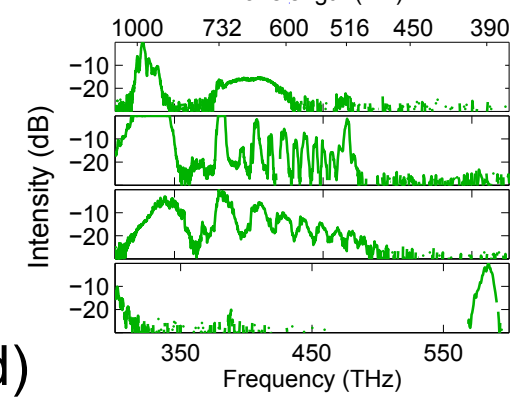

Figure 2.8: Summary of controlling nonlinear effects and spatiotemporal supercontnuum in GRIN MMF. (a) shows the basic experimental procedure, while (b) shows a typical spectrum, spanning the transparency of fused-silica (and requiring 3 separate spectrometers). (c) shows a few selected examples of different visible spectra obtained with different initial conditions. (d) shows dispersive waves resulting from space-time oscillations described in the previous sections.

experiments.

Thus, with these experiments and this work complete, I turned to the next chapter of my PhD with a goal of finding an even clearer explanatory order in the complexity, in an approach inspired by complexity science. To do this, I turned to normal dispersion fibers (to eliminate any possibility of MM solitons) and embraced the disorder and dissipation that had so far, by a dozen different means, been evaded. 


\section{CHAPTER 3}

\section{SELF-ORGANIZATION AND SPATIOTEMPORAL INSTABILITY IN NORMAL-DISPERSION MULTIMODE FIBERS}

Our work on multimode solitons and supercontinuum in the anomalous dispersion regime left many unanswered questions, tempting us to delve still deeper. As some of the commentary in previous chapters makes clear, this is precisely what we did. However as it goes, more often a good approach to an exploration is to begin with a broad survey. With this in mind, toward the end of 2015 my primary efforts turned to normal dispersion, and to trying to incorporate strong elements of disorder and dissipation. Roughly three factors influenced this decision. First, I wanted to do something within the world of multimode nonlinear fiber optics as far removed from the previous studies as possible. The normal dispersion regime eliminated the possibility of temporal solitons ${ }^{1}$. Second, since much of our motivation swirled around the development of high-power ultrashort-pulsed lasers based on multimode fiber, it seemed prudent to understand normal-dispersion multimode nonlinear fiber optics as a prerequisite to normal-dispersion multimode fiber lasers. As will be expanded on in the next chapter, normal dispersion lasers were expected to support much higher power because the dissipative solitons obtained in normaldispersion lasers are chirped (so the intracavity peak power is lower) and exist with larger roundtrip nonlinear phase shifts than solitons of the anomalous dispersion regime (so the absolute peak power in the cavity can be higher)[47].

Lastly, in 2016 Zhanwei Liu discovered a green (532 nm) Q-switched ns laser in our laboratory, and immediately saw it could be used for MMNLFO exper-

\footnotetext{
${ }^{1} \mathrm{~A}$ pretty notable exception here are dissipative solitons discussed in the next chapter, but these require more than just a passive fiber.
} 
iments. This odd discovery fit well, since the shorter wavelength meant that disorder within fibers would be more relevant (this can be seen most simply for the Rayleigh scattering from refractive index inhomogeneities, which scales with $\lambda^{4}$, but certainly applies in a lesser extent to other forms of disorder in the fiber).

\subsection{Nonlinear beam self-cleaning in multimode graded-index fiber}

In late 2015, Katarzyna Krupa and co-workers in France (principally Limoges at the time) told us of experiments they had recently performed, observing a non-dissipative nonlinear beam cleaning, and a new kind of parametric instability in multimode GRIN fiber, resulting from the self-imaging property of a parabolic waveguide (later called geometric parametric instability, GPI). Both effects have turned out to be very important core concepts in MMNLFO. These works were eventually published in 2016[44] and early 2017[48]. In a case of science progressing much faster than its published record, these effects turned out to be so easily-observable and important to MMNLFO in normal-dispersion GRIN fibers that both effects were soon observed in exploratory experiments being conducted in this regime in our own and other laboratories $[49,50]$. Beam cleanup especially turned out to be a surprisingly ubiquitous feature of many experiments in this regime[51,52,53,54,55,56] - a mysterious feature which in part motivated our work[57, 49] described in this chapter.

The signature of Kerr beam cleaning is shown in Fig. 3.1 [49]. A combination of modes is excited in the fiber, and at low power levels for which nonlineari- 
ties can be neglected, the output beam shows a speckled pattern resulting from the interference of many spatial modes. As the power is increased, this speckled pattern gradually changes into a beam profile dominated by the Gaussian fundamental mode of the waveguide.
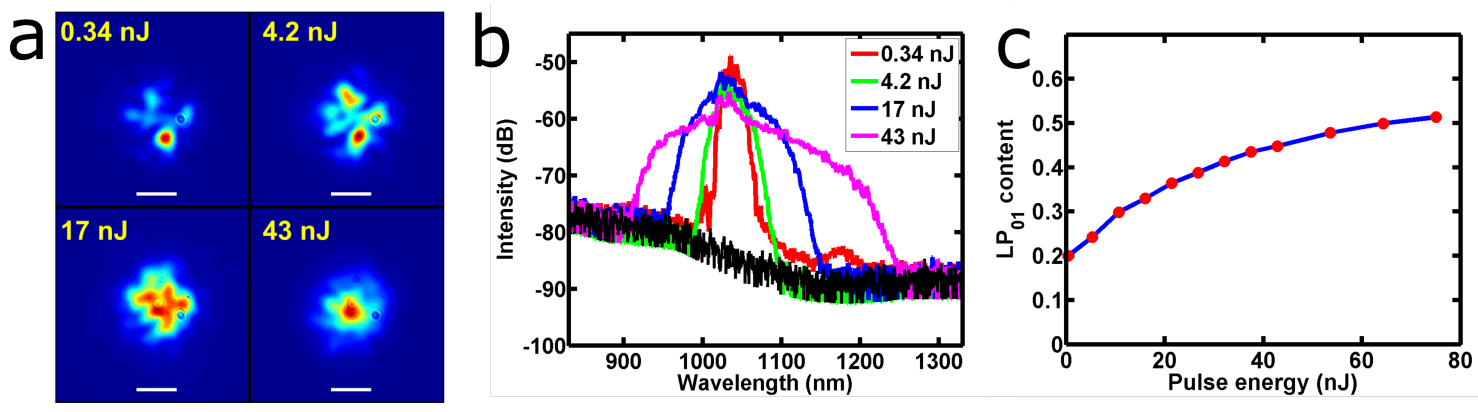

Figure 3.1: Kerr beam cleaning of femtosecond pulses. (a) shows the near-field beam profile for light at the end of a short GRIN fiber. (b) shows that spectral broadening is roughly symmetric, supporting the claim that the cleanup arises primarily through the conservative Kerr nonlinearity. (c) shows the fraction of energy in the LP01 mode for simulations with different pulse energy.

Kerr beam cleanup[48] occurs because, as multiple modes propagate in a nearly-parabolic GRIN fiber, the intensity oscillates at multiples of a single common period, the self-imaging period or pitch of the GRIN fiber, $P$. This intensity oscillation amounts to a longitudinal refractive index grating, which can provide the virtual momentum to phase-match four-wave mixing between different transverse modes, since those modes' propagation constants (i.e., their momenta) are of course separated by precisely integer multiples of $1 / P$. One would normally expect reciprocal exchange - that is, energy would oscillate back and forth between modes. However, this assumes that the rate of phase accumulation in the modes involved is unchanged by this exchange, and so the nonlinear index grating remains preserved throughout. This is not true: the fundamental mode accumulates nonlinear phase shift faster than other modes due to its high intensity, and high overlap with the other modes[48, 57]. For input powers 
roughly above some threshold, as energy flows into the fundamental mode, its rate of nonlinear phase accumulation can become out of phase with the modes from which energy was flowing. This terminates the phase-matched energy exchange, leaving energy permanently in the fundamental mode.

\subsection{Integrating the role of disorder and dissipation}

As was mentioned, several questions remained about beam cleanup. It has been natural to compare the process to Bose-Einstein condensation (BEC), which can be described for temperatures very close to $0 \mathrm{~K}$ by the Gross-Pitaevskii equation - which has precisely the same form as that describing nonlinear optical wave propagation in a GRIN fiber $[52,48,57,49]$. But in contrast to BEC, the essential features of beam cleaning seemed to be not only active when dissipation and disorder were added to the linear or nonlinear interactions between modes, but even enhanced and more easily observed. Indeed, it was previously wellknown that the Raman nonlinearity in GRIN fiber exhibited a beam cleanup process [51]. Thus, the question was 'Why is this self-organization of light into the fundamental mode such a universal feature of nonlinear light propagation in GRIN fiber?'

This question naturally matched the mission of exploring MMNLFO in a regime devoid of solitons, and with strong dissipation and disorder. We began with experiments. A 532-nm laser emitting ns-duration pulses was used so that, initially, modal and chromatic dispersion could be neglected, and so that disorder and dissipation (through mode-dependent loss, disorder-induced loss and Raman nonlinearity) were strong. A small-core GRIN MMF was used so that 
the total number of modes (55) was small enough that GMMNLSE simulations could be employed and expected to represent the physics reasonably well.

A set of typical experimental results is shown in Fig. 3.2. As the pulse energy (peak power) is increased, light self-organizes from an initially-multimode distribution into one strongly dominated by the fundamental mode. Both Raman and Kerr beam cleaning occur, as evidenced by the eventual very pure Gaussian beam profile, which could be observed across the spectrum at this point (if only Raman was responsible, only the red-shifted portion of the spectrum would be 'cleaned'[51]). Eventually, as the light has collected into the fundamental mode, sidebands become visible in the spectral domain. By measuring the spatiospectral profile and analyzing the sideband locations (Fig. 3.2), we attributed them to spatiotemporal, or intermodal, modulation instability.
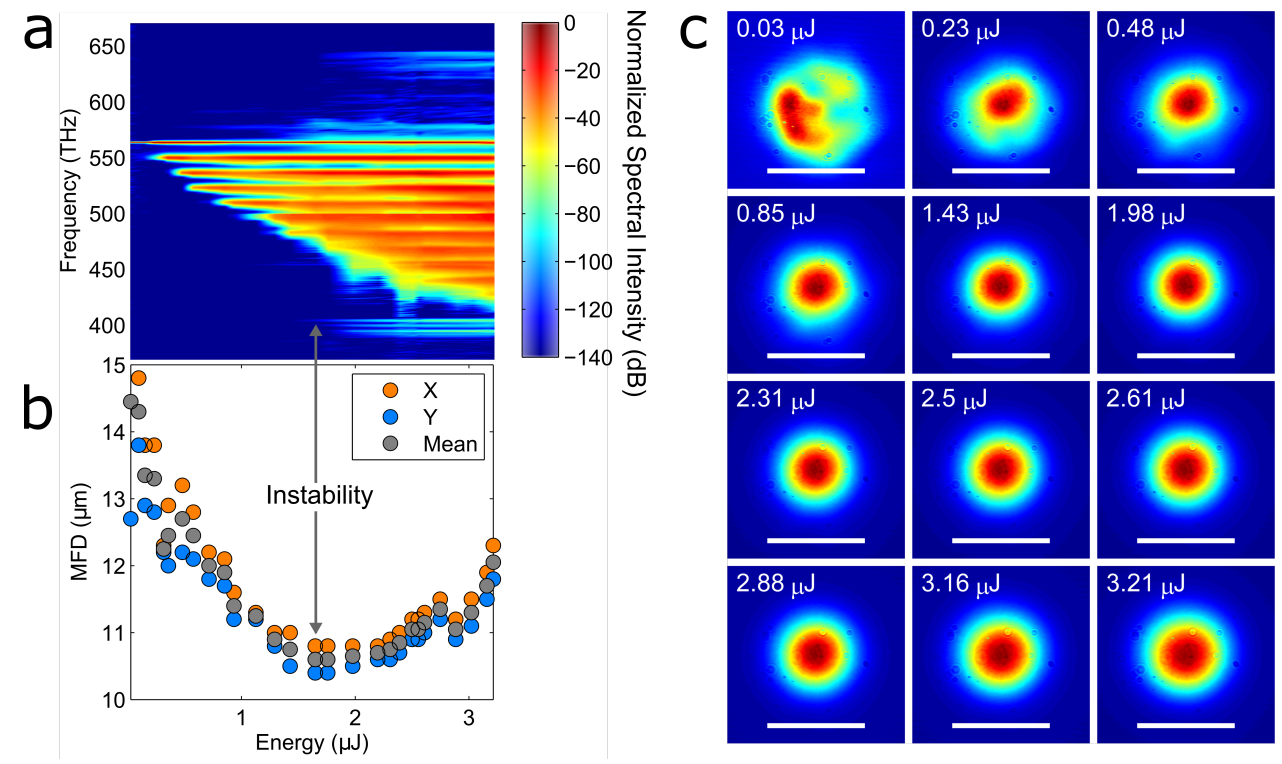

Figure 3.2: Experimental observation of self-organized instability in disordered, dissipative multimode graded-index fiber. (a)The spectrum and (b) the beam dimensions for increasing pulse energy launched into the fiber. (c) shows the near-field output beam profiles corresponding to the data in $a-b$.

This result evokes the concept of self-organized criticality, which ultimately 
inspired our theoretical analysis. Self-organized criticality refers to a general tendency of complex systems to self-organize toward a critical state, that is one which lies on the cusp of a phase transition[58,59]. In the case of the GRIN MMF, in the idealized description, this emergent behavior is the selforganization of the light in two-dimensions into a configuration which is maximally prone to a nonlinear instability that breaks the time-translation symmetry (that is, a phase transition) and ultimately gives way to spatiotemporal complexity (that is, a disordered multimode field in 3 dimensions).

\subsubsection{Coupled mode theory for describing the network}

To analyze different effects within the same framework, we employed coupledmode theory to produce a description of the complex coupling network that characterized all the interactions between modes. The point of this approach is not a quantitative or precise description - rather just to provide an intuitive framework for understanding emergent multimode phenomena - energy flow among the modes in space and frequency domains - in very complex scenarios where even the most efficient numerical simulations would be impractical (not to mention low on insight due to their complexity). We were able to verify this approach by comparing it to numerical simulations in limiting cases.

Before exploring the coupled-mode network, it is worth contextualizing and justifying our theoretical approach. Coupled-mode theory[61, 62] can, of course, be used to obtain 'rigorous' descriptions of nonlinear optical processes. Coupled-mode theory, in some sense, is the predominant theory of perturbative nonlinear optics, for example as the MMNLSE, or coupled-NLS descriptions 

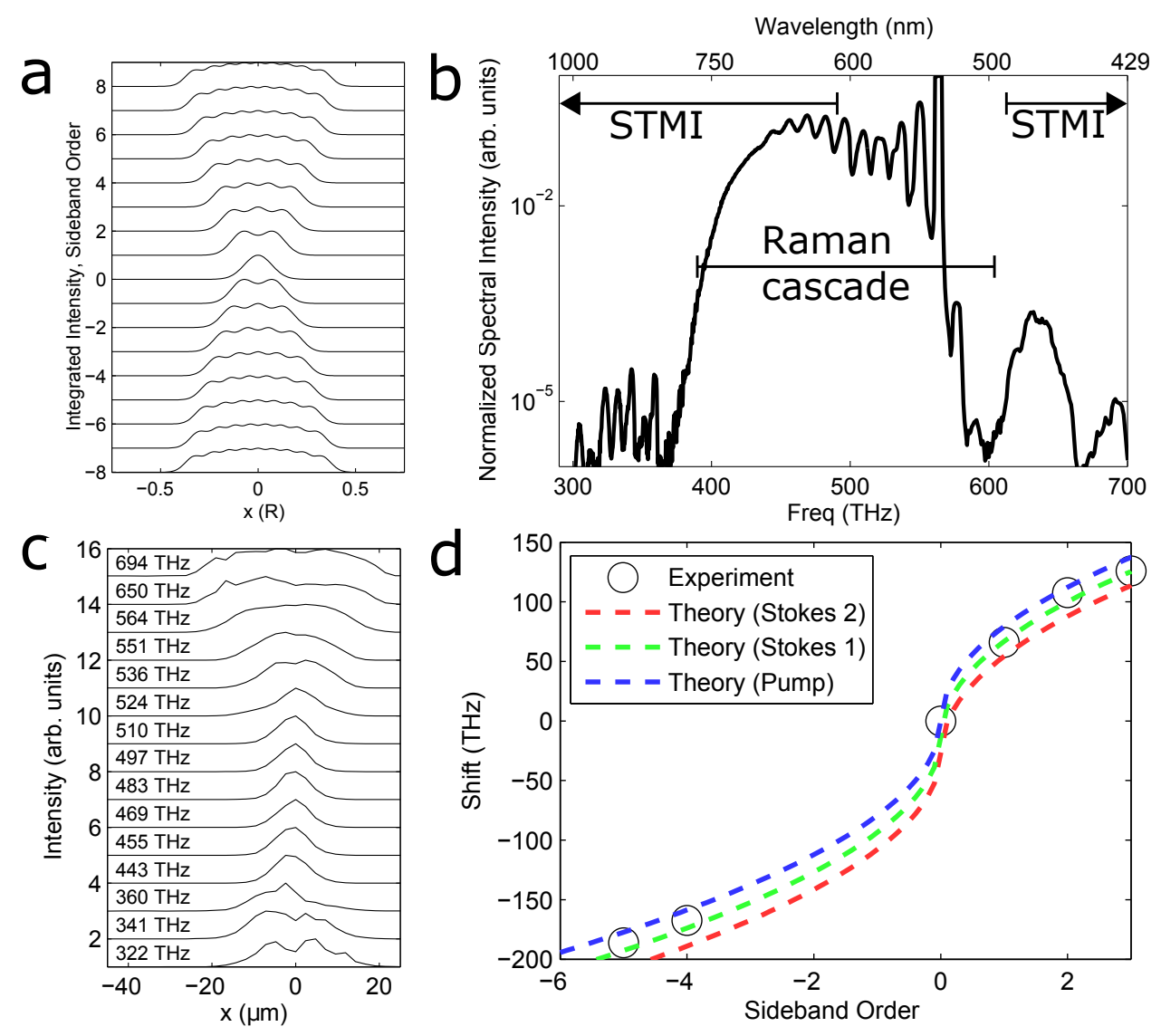

Figure 3.3: Spatiotemporal instability in multimode graded-index fiber. (a) shows the theoretically-expected spatiospectral profile for spatiotemporal modulation instability of the fundamental mode[60]. (b) shows the spectrum at the highest pulse energy, indicating the Raman cascade and the region where STMI sidebands are generated. (c) shows the measured spatiospectral profiles at the indicated frequencies, which replicate the expected hyperbolic spatiospectral shape. (d) shows the sideband shift measured, and the phase-matching curves, calculated for the pump at $532 \mathrm{~nm}$, as well as the two strongest Raman sidebands.

of four-wave mixing[13] or three-wave mixing[15]. However, without making certain approximations (for example, undepleted strong pump wave(s)), reducing the number of coupled modes (and the dimension of the coupling coefficients) to 2 is not possible. While the 3 - and 4-wave descriptions are numerically tractable, they lose much of the intuitiveness of 2-wave descriptions that apply to linear mode coupling. We can think of our approach in this chapter as taking 
a step backwards in a derivation of the MMNLSEs to where, despite not being numerically-tractable, all the mode-coupling processes appear in a similar way, making the interactions of these processes easier to understand intuitively. Thus, it is a technique for extracting low-dimensional intuition (but not necessarily quantitative answers or numerical simulations) from the more complex 4-wave description of the GMMNLSE, simply by looking at the equations differently.

A sketch of this complex coupled-mode network theory is as follows. For a fiber with eigenmodes $\phi_{i}$, the coupling (i.e., energy exchange) between any two modes, $\phi_{l}$ and $\phi_{m}$ can be understood through perturbation theory. The coupling coefficients are given by:

$$
\begin{aligned}
C_{l m}(z) & =\frac{k_{o}}{2 n_{e f f}} \frac{\left.\int_{-\infty}^{\infty} \int_{-\infty}^{\infty}\left[n_{o}^{2}(x, y, z)-\left(n_{o}(x, y, z)+\Delta n(x, y, z)\right)^{2}\right] \phi_{l}(x, y) \phi_{m}^{*}(x, y) d x d y\right]}{\sqrt{\int_{-\infty}^{\infty} \int_{-\infty}^{\infty}\left|\phi_{m}(x, y)\right|^{2} d x d y \int_{-\infty}^{\infty} \int_{-\infty}^{\infty}\left|\phi_{l}(x, y)\right|^{2} d x d y}} \\
& \approx K \int_{-\infty}^{\infty} \int_{-\infty}^{\infty} \Delta n(x, y, z) n_{o}(x, y) \phi_{l}(x, y) \phi_{m}^{*}(x, y) d x d y
\end{aligned}
$$

where $\Delta n(x, y, z)$ are the perturbations to the refractive index relative to the ideal index profile $n_{o}(x, y)$ for which the modes $\phi_{i}(x, y)$ were calculated. It is useful to write $\Delta n(x, y, z)=\Delta n(x, y) f(z)$. Here, $\Delta n(x, y)$ describes the overlap between the modes, while the longitudinal component $f(z)$ of the perturbation describes the phase-matching. The evolution of the amplitude of the field, $a_{m}(z)$ in the mode $\phi_{m}$ is given by $($ for $l=1, \ldots, M)$ :

$$
\frac{d a_{l}(z)}{d z}=-i \beta_{l} a_{l}+\sum_{m}^{M} C_{l m}(z) a_{m}
$$


Considering the coupling between two isolated modes, one can show $[62,61]$ that the amplitude of an initially weakly-occupied mode from a stronger one through a distance $L$ is

$$
a_{m}(L)=\int_{0}^{L} C_{m l}(z) e^{-i\left(\beta_{l}-\beta_{m}\right) z}
$$

Thus, the amplitude coupled is proportional to the coupling coefficient, and to the Fourier transform of the perturbation's longitudinal component, $F(\delta k)=F T[f(z)]$ evaluated at the propagation constant difference over a distance $L$. Thus, the periodicity of $f(z)$ influences what coupling processes are efficient. If $F(\delta k)$ is large at a value of $\delta k=\beta_{l}-\beta_{m}$, then provided the modes have some overlap (the integral Eqn. 3.1 is large) coupling between the modes will be strong. I like to think of $F(\delta k)$ as representing the reservoir of momentum provided by the medium perturbations to facilitate interactions between modes with different momenta.

This model can be extended to nonlinearity through $\Delta n(x, y, z)=n_{2} I(x, y, z)$ where $I(x, y, z)$ is the intensity of the MM field. While Eqn. 3.1 applies only to a single-frequency, we can also use the basic results to understand the phasematching that underlies multi-frequency four-wave mixing processes.

\subsubsection{Application to beam self-cleaning with disorder and dis- sipation}

The motivation to apply this approach is to understand the nonlinear attractor behavior of beam cleanup in GRIN MMFs. That is, why it occurs for a generic 
initial condition, and to understand how disorder and dissipation in the fiber affect it. We were able to show that, on average, there is a flow of energy from higher-order to low-order modes, culminating in the fundamental mode, and that weak disorder and disspation enhance beam cleanup, making it occur for a much broader range of initial conditions and power levels than would otherwise occur.

To show this, we considered what the average mode-coupling network would look like across many random excitations of the fiber, or for a typical excitation. This procedure shows that, on average, the coupling coefficients for nonlinearity-induced energy exchange favor the fundamental mode and, moreover, that there is a systematic bias toward lower-order modes. In other words, provided phase-matching, this bias indicates that energy may be expected to flow from higher-order modes to low-order modes, and in particular to the fundamental mode, because of nonlinearity-induced mode coupling.

All modes in GRIN are either quasi-degenerate $\left(\delta k=\beta_{l}-\beta_{m} \approx 0\right)$ or are separated by an integer number of the self-imaging wavevector (that is, $\left.\delta k=\beta_{l}-\beta_{m}=n / P\right)$. Thus, the beating between an arbitrary superposition of modes in the fiber produces a perturbation whose longitudinal component, $F(\delta k)$ has a large amplitude at $\delta k=n / P$ as well as around $\delta k=0$. Thus, from this 'reservoir of momenta', phase-matching is in general assured for all mode coupling processes. As a result, the bias of the mode-coupling coefficients is relevant, and thus for the majority of initial conditions, energy flows into low-order modes with increasing nonlinear index perturbation or propagation distance. This explains the ubiquity of the self-cleaning behavior in absence of disorder and dissipation. 
Dissipation and disorder, however, can be shown to enhance the cleanup (Fig. 3.2.2). They are moreover especially relevant at lower powers where the bias of the nonlinear coupling is only weak. This can be understood on first intuition as similar to how increased temperature may allow a system to more rapidly find equilibrium, by exploring phase space more quickly and thoroughly.

Dissipation affects both nonlinear and linear coupling. For the former, dissipation within the nonlinear coupling is responsible for a non-reciprocal selfcleaning process, Raman beam cleanup [51]. Raman beam cleanup originates from the same coupling bias to low-order modes that accounts for Kerr beam cleaning. Since the process is dissipative (it involves energy being lost to a phonon), energy transfer is a unidirectional, irreversible flow rather than the average flow tendency that occurs for conservative nonlinearity. For linear coupling, dissipation arises from coupling to cladding modes. Higher-order modes are more lossy in the fiber because they are more easily coupled to the cladding modes by disorder within the fiber. This preferential loss to higher-order modes means that even if all modes are equally excited, the lower-order modes soon have higher amplitude. This in turn enhances the bias of the nonlinear coupling coefficients.

Finally, disorder plays a somewhat more subtle role in self-organized beam cleaning. An intuition for this effect is that disorder tends to cause diffusion of energy among higher-order modes, and thus helps to direct the distribution of modes towards the average distribution of modes for which the bias to low-order modes occurs. This is the 'temperature/noise helps a system explore phase space' concept. More specifically, disorder introduces symmetry- 

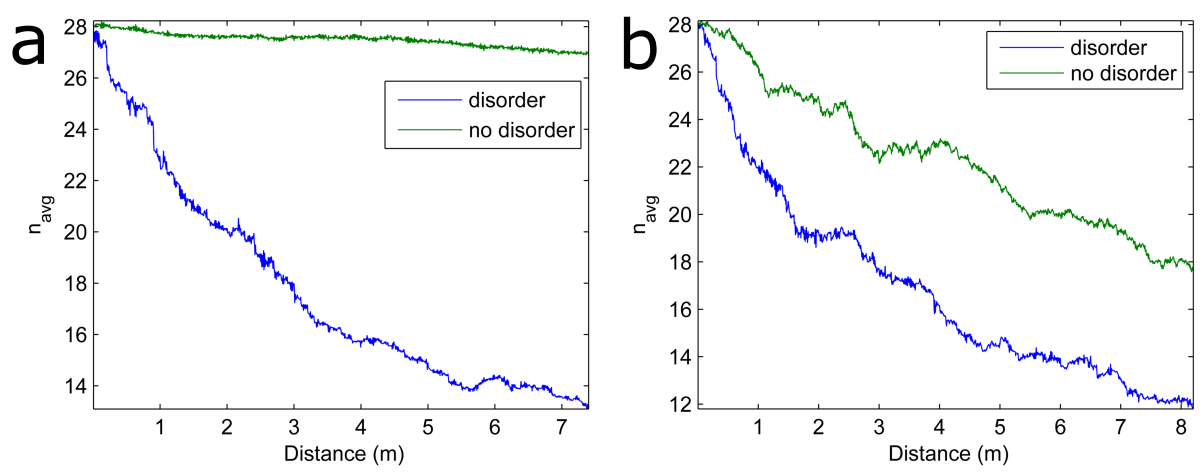

Figure 3.4: Effect of disorder on self-organized energy flow into lower-order modes in GRIN fiber. Simulations of monochromatic light propagation in a nonlinear GRIN fiber with and without realistic fiber disorder. (a) shows the average mode number, $n_{\text {avg }}=\sum_{m=1}^{M} m E_{m}$, where $E_{m}$ is the energy in mode $m$, for a $10 \mathrm{~kW}$ total power. (b) shows the same for $50 \mathrm{~kW}$ total power.

breaking perturbations to virtually all modes, and thus acts to seed nonlinear instabilities[63] and other disordered coupling processes. This seeding of the instability dramatically lowers their threshold, and in turn the threshold for selforganized beam cleaning toward the fundamental mode. Together, these processes make sure that even if a high-order mode becomes relatively dominant (and so the nonlinear coupling coefficients will at that instant exhibit a strong bias towards it), it is nonetheless unstable and will break up into a many-mode superposition, which can through the mechanisms described above exhibit selforganized energy flow into the fundamental mode.

\subsection{Multi-frequency multimode coupling network}

The nonlinear beam cleanup represents a flow of energy in 2D (that is, between modes at a single frequency or within a narrow range of frequencies) into a spatiotemporal unstable configuration in space. That is, it is self-organized criticality of a time-symmetric field into a spatiotemporally-disordered one. 
To see this, we need to add multiple frequencies to our mode coupling network mode. To describe multi-frequency mode coupling, we let $\delta k=\beta_{l}\left(\omega_{l}\right)$ $\beta_{m}\left(\omega_{m}\right)$ and, as a second constraint, enforce pairs of couplings for which energy conservation is ensured. This can be thought of as a continuation of the cohesive framework so far considered at one frequency into multiple frequencies. Technically it is rigorously correct with the constraint imposed, but this is not really our intent.

\subsubsection{Geometric parametric instability}

In the case of geometric parametric instability[64, 44], a continuous wave field in a superposition of modes generates spectral sidebands which are composed of a field in the same (or nearly the same) superposition of modes. Since a superposition of modes produces a longitudinal index perturbation $F(\delta k)$ which has large amplitudes at integer multiples of $1 / P$, the perturbation can phasematch coupling between the same mode at two (four considering the energyconservation constraint) different frequencies, for which $\delta k=\beta_{l}\left(\omega_{l}\right)-\beta_{l}\left(\omega_{m}\right)=$ $n / P$.

\subsubsection{Spatiotemporal modulation instability}

A related modulation instability also occurs in GRIN fiber, for which the sideband positions are precisely the same as for GPI, but which has a fundamentally different origin. As is suggested by its name, in spatiotemporal modulation instability (STMI), phase-matching occurs through spatial, or modal, dispersion 
matching temporal dispersion $[65,66,60]^{2}$. With STMI, $\delta k=\beta_{l}\left(\omega_{l}\right)-\beta_{m}\left(\omega_{m}\right)$ is close to 0 . Even though $\omega_{l}$ and $\omega_{m}$ are very different, in different modes the propagation constants may be the same (i.e., $\beta_{l}\left(\omega_{l}\right) \approx \beta_{m}\left(\omega_{m}\right)$ ), and so nonlinear coupling will occur provided there is some spatial overlap of the modes.

Hence, while GPI is an instability which applies to a multimode field which produces an index perturbation, a nonlinear index grating, whose $F(\delta k)$ has large amplitudes at integer multiples of $1 / P, \mathrm{STMI}$ applies to nearly single-mode conditions, such as when all the energy has flowed into the fundamental mode, and where the nonlinear index perturbation has an $F(\delta k)$ with high amplitude only near 0 (since no or very little mode-beating is occurring).

GPI and STMI represent limiting scenarios for multi-frequency nonlinear coupling, and of course it is possible for processes which are part-GPI and partSTMI to occur. In other words, for a given intermodal, multi-frequency process, the phase-matching may be in part completed by a nonlinear index grating written by a multimode field, and in part by the different dispersion of different modes.

\subsection{Self-organized instability}

Finally, we can understand the self-organized criticality, or instability, that is the nonlinear attractor. Our analysis shows that energy flows into the fundamental mode, which is the most unstable configuration of the light when spatiotemporal effects are considered. Figure 3.4 shows a numerical verifi-

\footnotetext{
${ }^{2}$ While GPI is also clearly a spatiotemporal instability, it is nonetheless more instructive to emphasize the space-time matching in STMI, in order to differentiate it from GPI, which is phase-matched by the nonlinear index gratings.
} 
cation of this result. It is, however, intuitive from the previous discussion why the fundamental mode should have the highest instability, and thus represent the two-dimensional configuration of light which maximizes the timetranslation-symmetry-breaking and the development of spatiotemporal complexity through multiple multi-frequency, multimode coupling processes. First, the fundamental mode has, on average, the highest intensity, and the highest overlap with all other modes. Second, it is the least affected by disorder, and loss induced by disorder, so nonlinear coupling processes may proceed with relatively minimal interference from disorder.

Why do the nonlinear dynamics of light in disordered, dissipative GRIN MMFs exhibit this universal pattern of self-organization toward an instability? One answer is that it is simply because of the complex multi-frequency mode coupling network's topology, as outlined above. This is just how energy is expected to flow through the network. A more general observation appeals to thermodynamics. That is, it is intuitive that the state of maximal spatiotemporal complexity is the highest entropy configuration of the field, given its degrees of freedom in space as well as in time (frequency). Thus, self-organized criticality here and in general is in some sense the statement that, in the interest of ultimately maximizing entropy, certain temporary self-organizations, even if they might represent 'momentary violations' of the second law, may be tolerated or even expected[67]. 


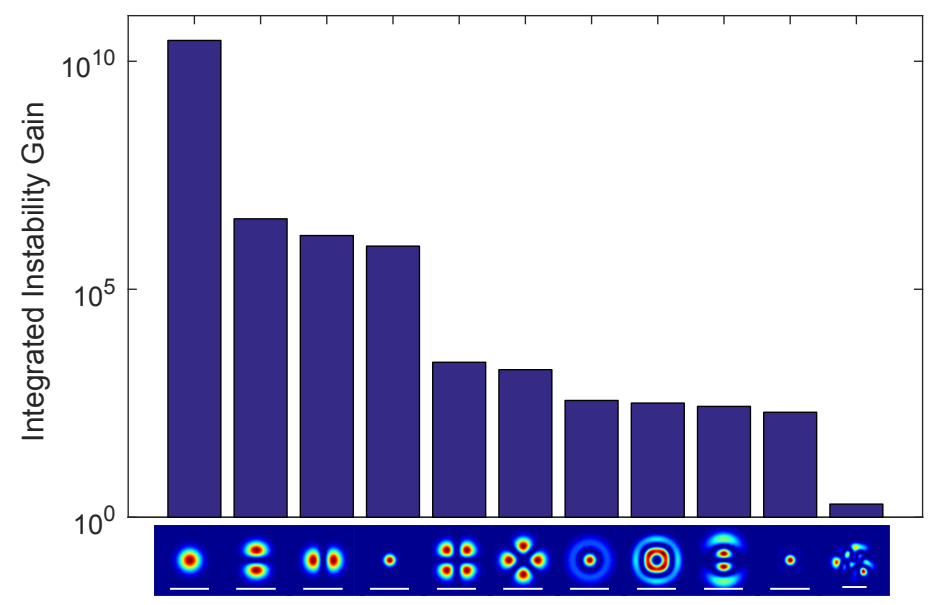

Figure 3.5: Spatiotemporal instability of different field distributions in GRIN fiber. For fixed peak power and the initial conditions shown at the bottom, simulations using the GMMNLSE were run, and the total gain (integrated over the band, and starting from quantum noise) of the first STMI/GPI sideband was calculated. The result shows that, as energy flows from an initially-MM field, such as the last condition on the right, into the fundamental mode, the last condition on the left, the instability of the field grows by roughly 10 orders of magnitude. 
CHAPTER 4

SPATIOTEMPORAL MODE-LOCKING AND MULTIMODE FIBER

LASERS

\subsection{Introduction}

The basic ideas underlying spatiotemporal mode-locking and multimode fiber lasers emerged very early on in my PhD. research. While it remains to be seen if these ideas should be considered 'good', or even if they can be considered scientific ideas at all, they fulfill at least one common quality of good ones: they are so obvious that a novice can not only understand them, but in fact may even be most likely to suggest them. This of course existed within the context of Frank's group being a group that researched high-power mode-locked lasers, which directly motivated explorations into multimode fiber [68], as outlined in Chapter 1 .

With these motivations in mind, in early 2015 Zhanwei Liu even performed a set of experiments attempting to observe mode-locking in multimode lasers. While fairly systematic and well-motivated, this work was guided only by our then-immature intuition about how the physics might play out, including a number of biases following from single-mode laser design. They produced rather discouraging results that soon caused it to be abandoned, and without clear conclusions.

Nonetheless, following the culmination of the works in the previous chapter, in late 2016 the multimode fiber laser seemed to be the point in the world of MM nonlinear waves most remote from what had so far been successfully 
mapped out. As I began to develop the theoretical tools to help inform eventual experiments, my attitude was emboldened when Walter Fu learned of an odd one-off custom fiber produced by Nufern, a partially graded-index, Yb-doped multimode fiber. Since the low modal dispersion of a GRIN fiber makes strong nonlinear coupling between short pulses in different modes easier, a GRIN gain fiber seemed to be a key requirement for a MM laser. For this reason it was something we had been, as research group, inquiring about for the previous years. While the fiber obtained is not a 'true GRIN' fiber in the sense that it does not have a parabolic profile, it does have lower modal dispersion than a typical MM gain fiber. This is in part due to a partially-graded profile, but also in large part simply because it is a highly multimode fiber. In contrast, fibers with only a few modes typically exhibit higher modal dispersion (and of course, in the limit of a continuous rod, the modal dispersion is negligible).

In this chapter, I will first review the modes of a multimode laser cavity. Then, I will introduce the general description of spatiotemporal mode-locking. Last, the first experiments and simulations on STML will be reviewed, and our current understanding of the physics will be summarized.

\subsubsection{Longitudinal and transverse modes in laser cavities}

A laser is formed by an optically-active medium with a population inversion, and some optical feedback mechanism that redirects stimulated emission from this medium back into it repeatedly; usually a cavity. The lasing modes of a three-dimensional cavity are $F_{n}(x, y, z)$, which are three-dimensional electromagnetic field patterns which have distinct oscillation (lasing) frequencies, $\omega_{n}$. 
Due to the non-Hermitian boundary conditions, the 3D modes are not orthogonal, instead the forward-traveling modes are biorthogonal with the backwardtraveling modes. However, in most cases of interest the modes represent a complete basis set such that the laser field can be described as:

$$
E_{\text {laser }}(x, y, z, t)=\sum c_{n}(t) F_{n}(x, y, z) \exp ^{-i \omega_{n} t}
$$

where the $c_{n}$ are complex numbers. When the $c_{n}$ have a well-defined, nontrivial (i.e., more than $1 c_{n}$ is non-zero) relationship that is stable over many times the laser's fundamental oscillation period, $n L / c$, the laser is spatiotemporally mode-locked.

In almost every mode-locked laser considered to date, the situation has been considerably simplified by making an additional restriction. By creating high loss to all but one transverse mode (usually the Gaussian fundamental mode), $F_{n}(x, y, z)=F(x, y) F_{n}(z)$. In other words, $F(x, y)$ is fixed, and the modes only vary along $z$; that is, they are only longitudinal modes. With this measure, modelocking is reduced from a $(3+1)$ to a $(1+1)$-dimensional problem. Very similar considerations apply to mode-locking in coherently-pumped optical resonators.

To begin, let us examine the mode resonances of a typical multimode laser cavity. In many situations of interest, we may write the modes of the 3D laser cavity as a composite function $F_{n}(x, y, z)=F_{q}(x, y) F_{p}(z)$. We can then write the resonant frequencies in terms of the longitudinal mode numbers $p$ and transverse mode numbers $q$ as $\omega_{p q}$ (generally there are two such numbers, but for compactness we will assume all combinations are represented by a list index q). The condition for resonance for a given mode is that the cavity, of optical 
roundtrip length $n L$, comprises an integer number of wavelengths:

$$
\omega_{p q}=\frac{2 \pi c p}{n_{p q}\left(\omega_{p q}\right) L}
$$

Examples of what these modes look like are shown in Figure. 4.1

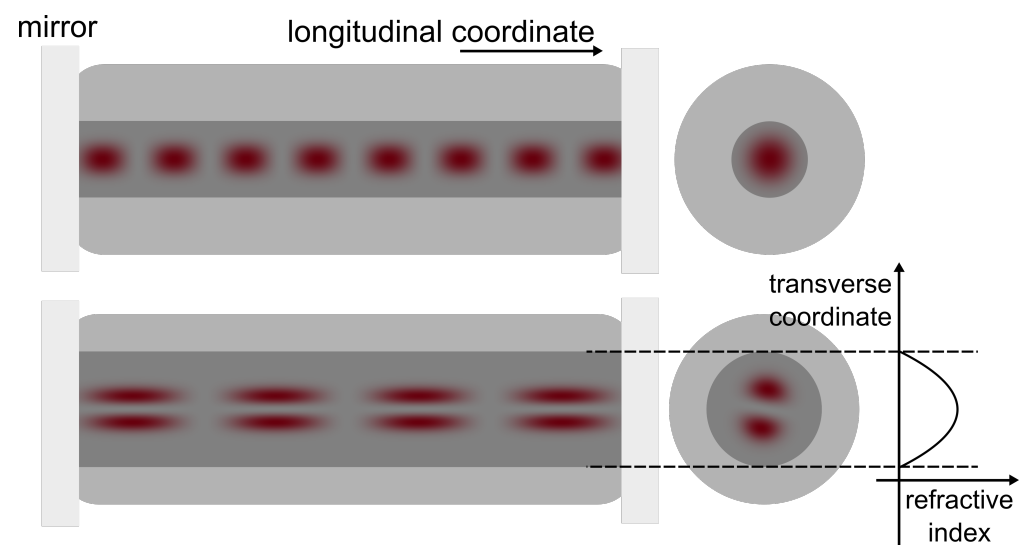

Figure 4.1: Examples of 3D modes of a multimode cavity. The top shows a single-mode fiber laser cavity, with a fundamental transverse mode and particular longitudinal mode intensity. The bottom shows a multimode fiber laser cavity, which exhibits in addition to the fundamental transverse mode, also many higher-order transverse modes (such as the LP11 shown) each with their own family of longitudinal patterns.

In the case of a multimode fiber laser, $n_{p q}\left(\omega_{p q}\right)$, are the effective indices ${ }^{1}$ for the given transverse mode at the frequency $\omega_{p q}$. For other resonators, the optical roundtrip length may need to be supplemented with mode-dependent geometric factors (i.e., the Guoy phase shifts). By substituting $n_{p q}\left(\omega_{p q}\right)=\beta_{p q}\left(\omega_{p q}\right) / \omega_{p q} c$ into Eq. 4.2, expanding the $\beta_{p q}$ up to second order in frequency, one can show:

$$
\omega_{p q}=\omega_{0}+\Omega_{p} \frac{\beta_{1}^{1}}{\beta_{1}^{q}}-\frac{\beta_{2}^{q}\left(\beta_{1}^{1}\right)^{2}}{2\left(\beta_{1}^{q}\right)^{3}} \Omega_{p}^{2}
$$

${ }^{1}$ The effective index is just the difference between the vacuum wavenumber $k_{o}=2 \pi / \lambda$ and the modal propagation constant, $\beta=n_{e f f} k_{o}$. 
where $\Omega_{p}$ is $2 \pi\left(p-p_{0}\right) / L \beta_{1}^{1}$ is the ideal angular free-spectral range (the freespectral range is $\Delta_{p}=\Omega_{p} / 2 \pi$ ) for the fundamental transverse mode family ( $q=$ 1), and where we are assuming for simplicity that the different transverse mode families have a common reference resonance, $\omega_{0}$. If we ignore the dispersion in the third term, Equation 4.3 shows that, for each transverse mode family $q$, the longitudinal modes correspond to a frequency comb with a spacing $\Omega_{p q}=$

$\Omega_{p} \frac{\beta_{1}^{1}}{\beta_{1}^{4}}$. For each such comb, the local free-spectral range, which is $\approx \frac{1}{2 \pi} \partial \omega_{p q} / \partial p$, varies linearly according to the chromatic dispersion of that mode family, $\beta_{2}^{q}$. For linearly-propagating fields inside the resonator, the phase shift per roundtrip is

$$
-\frac{d \phi_{p q}}{d T_{p q}}=\frac{\beta_{q}\left(\omega_{p q}\right) L}{L / v_{q}}=\beta_{q}\left(\omega_{p q}\right) v_{q} \approx \omega_{0 q}+\Omega_{p} \frac{\beta_{1}^{0}}{\beta_{1}^{q}}+\frac{\beta_{2}^{q}\left(\beta_{1}^{0}\right)^{2}}{2\left(\beta_{1}^{q}\right)^{3}} \Omega_{p}^{2}
$$

However, there is also dispersion of these resonances due to the dispersion of the frequencies and transverse modes. Figure 4.2 depicts qualitatively (i.e., exaggerating the dispersions) what the resonances from Eqn. 4.3 look like in a laser, assuming everything is linear.

\subsubsection{Spatiotemporal mode-locking}

But everything is not linear! When additional effects are introduced, including dissipative linear ones like spatial and spectral filtering, as well as nonlinear interactions between the modes, these resonances can shift into ordered frequency structures. Likewise, the multimode lasing field can shift from a complex 'continuous wave' lasing state with lots of complex mode beating into a circulating coherent MM pulse. As was stated earlier, (spatiotemporal) mode-locking corresponds to an electromagnetic field state where the modal amplitudes maintain a 


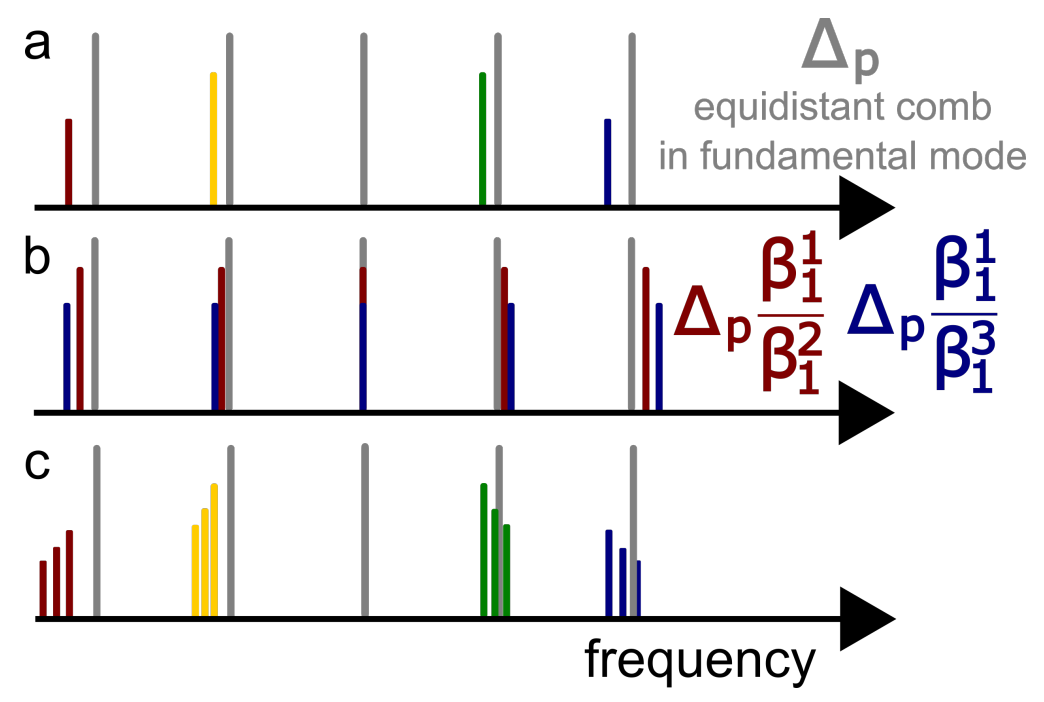

Figure 4.2: Conceptual depiction of lasing frequencies in a multimode laser cavity. (a) shows the effect of (normal) chromatic dispersion, which shifts the modes from an ideal equidistant frequency comb. (b) shows modal dispersion without chromatic dispersion. Each color represents a distinct transverse mode family, q. (c) shows a resonant mode spectrum considering both modal and (normal) chromatic dispersion.

fixed relationship. In the case of mode-locking in a single transverse mode with anomalous chromatic dispersion, nonlinearity (Kerr nonlinearity in the laser medium, as well as dissipative nonlinearity in the saturable absorber) cause phase modulations that cancel out the final term in Eqn. 4.3 above, resulting in the equally-spaced modes of mode-locked operation. For normal chromatic dispersion, spectral filtering is also important in the mode-locking process. When multiple transverse mode families are considered, the description is precisely the same, but we must generalize our language slightly. Processes which affect the lasing frequencies' self-organization include:

- Spectral, spatial, and most generally spatiospectral filtering. These introduce linear mode-dependent loss. Spatial and spatiospectral filtering may introduce dissipative linear coupling between modes.

- Linear mode coupling. Devices like Bragg gratings, or spatial phase 
masks like spatial light modulators, may couple together fields in different spatial modes with the same frequency (i.e., photons with distinct momenta but identical frequency). Disorder within the laser cavity may also create mode-coupling of this kind. Linear mode coupling also occurs in a spatial filter.

- Nonlinear mode coupling. The Kerr nonlinearity in the laser medium, and/or the dissipative nonlinearity in the saturable absorber cause longrange (nonlocal in the frequency domain, local in the time domain) coupling between distinct resonant modes. In addition, gain saturation can cause a (typically slower) interaction between modes. Last, modulation of the cavity by an active device like an electro-optic modulator may introduce local (in the frequency domain, nonlocal in the time domain) coupling between nearby resonant modes.

The simplest form of spatiotemporal mode-locking is just mode-locking in a single transverse mode. In this case, the linear resonances are:

$$
\omega_{1 q}=\omega_{0}+\Omega_{p}-\frac{\beta_{2}^{q}}{2 \beta_{1}^{1}} \Omega_{p}^{2}
$$

In this situation, one can see that lasing at each frequency oscillates at a resonance frequency that makes up an equally-spaced comb of frequencies, except that each is shifted slightly due to the dispersion. This dispersion shift means that the net phase each round trip, $\frac{d \phi_{1 q}}{d T_{1 q}}$ varies across the spectrum. In other words, the field disperses chromatically in time. When mode-locking occurs, nonlinear interactions between the modes, as well as possibly through linear dissipative elements like a spectral filter, cancel the final term, leaving a fre- 
quency comb. In this case, the phase acquired by the laser field in each mode over one round trip is:

$$
-\frac{d \phi_{1 q}}{d T_{1 q}} \approx \omega_{0 q}+\Omega_{p}
$$

Eqn. 4.6 simply shows that each mode's phase evolves only according to its oscillation frequency, and so the field does not disperse but is instead constant from round trip to round trip. It is, in other words, a pulse that is the same every round-trip. Another way to see this it to note that the Fourier transform of a comb of equally-spaced oscillators (frequencies) is a train of pulses, equallyspaced in time.

The simplest form of spatiotemporal mode-locking beyond mode-locking in a single transverse mode family is depicted in Fig. 4.3a, where the lasing frequencies of each transverse mode family coalesce into a common frequency comb. For the case of normal chromatic dispersion that we have primarily explored so far, this form of mode-locking is the most common. As the above discussion suggests, there are many other possibilities, such as the situation depicted in Fig. 4.3b. To describe these, however, will require a more sophisticated framework than the deliberately-simplified one we have considered so far.

In this 'next simplest STML' case, the lasing frequencies correspond (through the Fourier transform) to a temporal pulse train at a single repetition rate, $\Delta_{S T M L}$. In a reference frame of a pulse in the fundamental mode, and with a center frequency $\omega_{o}$, the fact that the mode-locked lasing frequencies are not the same as mode-locking in the fundamental mode means that the STML pulse moves at a finite velocity relative to the fundamental mode. We can write the resonant 
frequencies in the reference frame of the fundamental mode cavity as:

$$
-\frac{d \phi_{p q}}{d T}=\omega^{S T M L}=\omega_{0}+\Omega_{p}+\alpha \Omega_{p}=\omega_{0}^{S T M L}+\Omega_{p}^{S T M L}
$$

where the use of $T$ rather than $T_{p q}$ or $T_{q}$ is noteworthy, and where $\alpha$ is a parameter for the non-zero velocity of the pulse in the reference frame of the fundamental mode at the center frequency. Equating the previous two, we find $\alpha=\left(\beta_{1,0} / \beta_{1, S T M L}-1\right)$. This is just a change to the static reference frame of the STML pulse. What determines the STML repetition rate? So far, it turns out to not always be simply the weighted average of the repetition rates of the constituent transverse mode families. However, this is nonetheless a good initial guess.

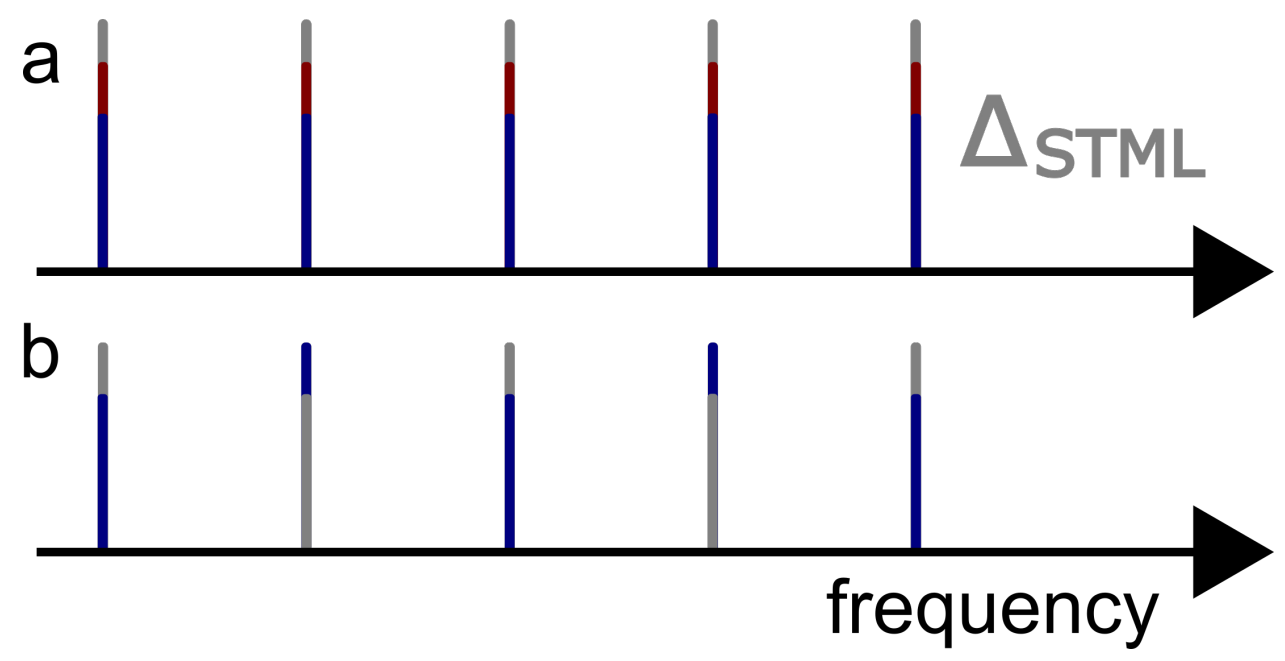

Figure 4.3: Examples of spectra of STML lasers. In (a), the resonant modes of each transverse family shift into a common, equidistant frequency comb, while in (b), two modes form a more complex comb with a larger unit cell. Many much more complex spatiotemporally mode-locked states are possible in principle.

All this introduction shows that STML can be thought of as a generalization of mode-locking in a single-transverse mode. In a single-mode mode-locked laser, nonlinear and linear coupling between modes can counteract dispersion, 
shifting the lasing frequencies into a phase-locked frequency comb. In a MM mode-locked laser, everything is the same, except that the modes are $3 \mathrm{D}$, dispersion includes both chromatic and modal dispersion, and the nonlinear and linear coupling occurs not only between longitudinal modes but also transverse modes. In conventional mode-locking, nonlinear and linear interactions between modes must counteract the chromatic dispersion of the cavity to form a self-organized, phase-locked frequency comb. In a MM mode-locked laser, the same is true in the more general sense that interactions and dispersions are spatiotemporal. Thus, a first intuition about the design of a laser for STML is that the spatial dispersion (modal dispersion) should be small, or at least comparable to the chromatic dispersion that we already know can be counteracted by nonlinearity to cause mode-locking.

To really study this in more detail requires a much more advanced model. Let's take a look at what a MM laser cavity in which STML may occur looks like. This is not the most general depiction of an STML cavity, but it is prototypical enough to begin with. Figure 4.4 shows a conceptual schematic of this protocavity.

Numerically, this is solved by taking an electric field envelope $A(x, y, t ; z)$, propagating it through the nonlinear, dispersive gain medium, applying a saturable absorber transfer function, then dividing the field into output-coupled and feedback components, then filtering with some combination of spatial and spectral filters, and then repeating.

The nonlinear, dispersive gain medium could, for example, be one multimode gain fiber. It could be a rod of doped glass, a combination of multimode and single mode fibers, or even just single-mode fibers (in which case it would 


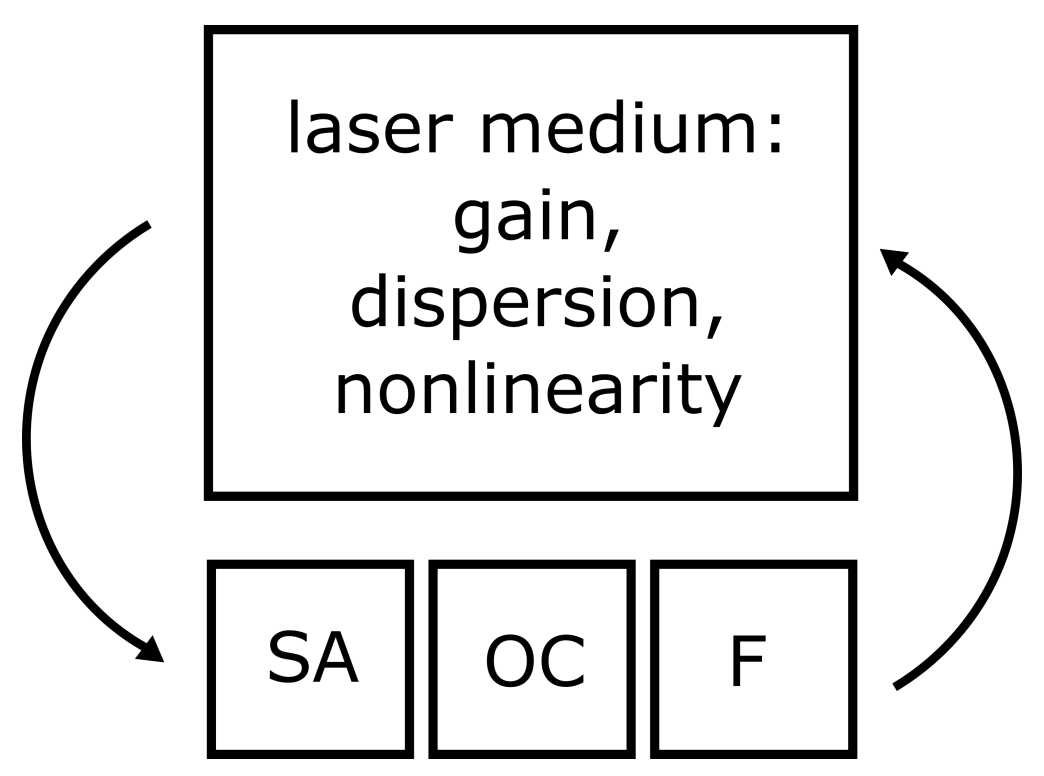

Figure 4.4: Conceptual depiction STML laser cavity. The cavity comprises a nonlinear, dispersive gain medium, a saturable absorber (or something to act like one), an output coupler, and a filter (spatial, spectral, and/or spatiospectral).

be a typical mode-locked fiber laser cavity). The propagation in this medium can be described by:

$$
\begin{aligned}
\partial_{z} A(x, y, t ; z)= & \frac{i}{2 \beta\left(\omega_{o}\right)} \nabla_{T}^{2} A-i \frac{\beta_{2}}{2} \partial_{t}^{2} A \\
& +i \frac{\beta\left(\omega_{o}\right)}{2}\left[\left(n(x, y, z) / n_{o}\right)^{2}-1\right] A+i \frac{n_{2} \omega_{o}}{c}|A|^{2} A \\
+ & \frac{g_{o}(\omega)}{1+\int_{t_{\max }}^{t_{\max }}|A(x, y, t ; z)|^{2} d t / J_{\text {sat }}} A
\end{aligned}
$$

Where the equation is very similar to Eqn. 1.1 except the last term, representing the slowly-saturating gain, where $g_{o}(\omega)$ is the frequency-dependent smallsignal gain and $J_{\text {sat }}$ is the saturation fluence (energy density) of gain medium. The integral's bounds here are over the simulation time window, which is a window of time around the pulse typically shorter than the round trip time. The limits of the approximation will be discussed later. The parameters of the gain may also be functions of space (for example, to reflect an inhomogeneous 
dopant or pump distribution).

Propagation through the saturable absorber, filters, and output coupler can be described by spatiotemporal transmission functions:

$$
\begin{gathered}
A(x, y, t ; z) \rightarrow A(x, y, t ; z) \sqrt{1-\frac{\alpha_{o}}{1+\frac{|A(x, y, t ; z)|^{2}}{I_{s a t}}}} \\
A(x, y, t ; z) \rightarrow \sqrt{1-O C} A(x, y, t ; z) \\
A(x, y, \omega ; z) \rightarrow F(x, y, \omega) A(x, y, \omega ; z)
\end{gathered}
$$

Here, $\alpha_{o}$ is the modulation depth, $I_{s a t}$ is the saturation intensity of the saturable absorber, $O C$ is the output coupling ratio (so $\sqrt{O C} A(x, y, t ; z)$ is the output field), and $F(x, y, \omega)$ is the filter transmission function. While the last coordinate in the filter transmission function can only be $\omega$ (we cannot filter in time passively), the first two coordinates may also be $k_{x}$ and $k_{y}$, if we are performing filtering in the far-field. In many situations, it is convenient to perform a change of basis on the above equations, and to propagate in a transverse mode basis in some or all of the stages of the cavity.

\subsubsection{Understanding STML with a first example}

Fig. 4.5 shows an example of a stable, STML pulse. In this cavity, a small spatial filter (in the near-field) is used to force mode-locking into radially-symmetric 
modes. The size of the spatial filter, $20 \mu \mathrm{m}$, is chosen to be smaller than the fundamental mode size, roughly $35 \mu \mathrm{m}$. While this is not always required to observe STML states of the kind shown in Fig. 4.5, it is a reliable route because it ensures that the lowest-loss configuration of modes is a superposition of multiple transverse mode families. This concept of 'lowest loss configuration' is a useful one in understanding STML in general: the superposition of 3D modes that minimizes the round-trip loss through the cavity will tend to experience the highest gain, since the gain amplifies all modes exponentially. The available total gain is limited, and so with this exponential advantage, the superposition of modes which obtains the maximum round-trip gain will tend to extract all the available gain and all other possible lasing configurations are suppressed. The presence of nonlinear interactions and feedback, such as the saturable absorber, imposes a phase-sensitivity to the mode interactions, and means that the ideal superposition of modes is not just a distribution of energy in modes (i.e., mode intensities), but actually a specific set of complex 3D mode amplitudes (that is, having both well-defined magnitudes and phases).

This behavior resembles passive mode-locking by Kerr lensing, as is commonly employed in modern Ti:sapphire lasers[69, 70, 71]. Since the modulation resulting from Kerr lensing (KLM) tends to be very small, the Ti:sapphire laser is only very weakly operating in what could be considered multiple transverse modes of the 'cold', or linear resonator. Most features of the KLM Ti:sapphire laser differ from the typical scenario in MM fiber lasers. MM fiber lasers are significantly more nonlinear, strongly-dispersive and dissipative, the latter being a combination of high gain, loss, strong spectral and spatial filtering and much stronger total saturable absorption. Nonetheless, even if very slightly, this type of mode-locking is absolutely a meaningful (i.e., more than 1 transverse mode 

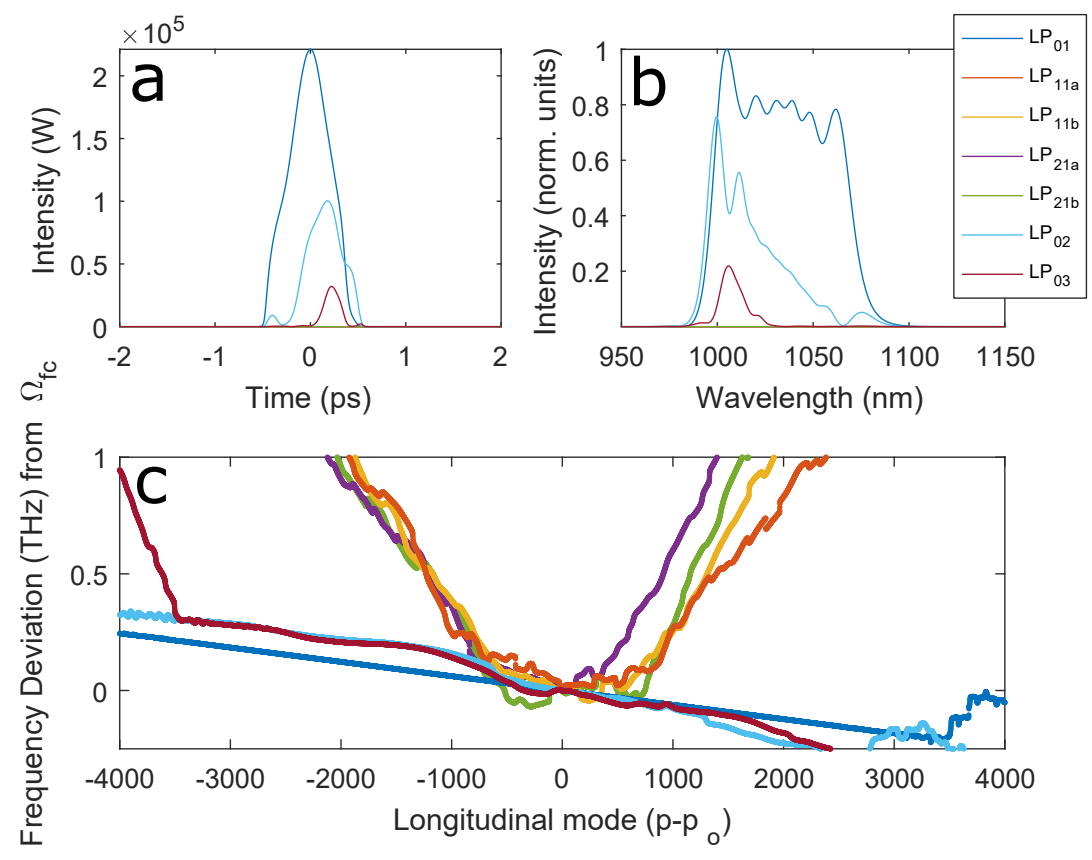

Figure 4.5: Numerical example of STML in multimode fiber laser. One of the most common types of STML observed is pulse in which multiple transverse families lock together to form a steady-state, multimode pulse that is constant from round-trip to round-trip. (a) shows the steady-state, mode-resolved pulse, while (b) shows the spectrum. (c) shows the steady-state round-trip phase shift of different longitudinal modes in each transverse mode family (i.e., the lasing frequency shift from an ideal frequency comb in the fundamental mode). This shows that modes that are locked into the pulse exhibit a common, stable frequency comb, where the linear deviation corresponds to a shift of the repetition rate (frequency comb separation) from what would be expected for modelocking in the fundamental mode (which would be a horizontal line). Unlocked modes propagate linearly, so the lasing frequencies exhibit a quadratic deviation from the ideal frequency comb in the fundamental mode.

family) form of STML.

The spatiotemporal mode-locking self-organization process is in some sense analogous to evolution by natural selection. The effect of different genes is 'phase-sensitive' and 'nonlinear' in that a given organism's characteristics do not result merely from the independent action of its distinct genes, but also the 
sequence and combination (in general in a nonlinear fashion). An organism's fitness determines its rate of exponential reproduction of said genes. Given the finite supply of energy available to the population, in a fixed environment evolution will proceed until the dominant organism represents the optimally-fit solution to the specific constraints of the environment, and within the universal constraints of physics.

It is tempting to get carried away with this analogy, and with it acknowledged as such, let us briefly do just that. For one, the analogy vividly illustrates some of the broader potential for for MM mode-locked lasers to facilitate the automatic design of physically-optimal 3D electromagnetic fields that maximize multiple simultaneous objectives, and in a natural and smooth way. In the MM laser, each 'generation', furthermore proceeds in mere nanoseconds, with steady-state being reached on microsecond timescales. In our real MM laser, this represents a nonlinear optimization of 100s of millions of complex amplitudes (a number that is comparable to the human genome's roughly 3 billion base pairs).

This example features a typical pulse evolution within the cavity (Fig. 4.6). As the pulse forms from noise, the lasing field exhibits a complex evolution in space and time both, a spatiotemporal (3D) transition to mode-locking. However, once the optimal pulse is found, a periodic steady-state evolution is observed. In this evolution, the distribution of energy among the transverse modes is relatively constant (Fig. 4.6b), even though the modes experience significant gain (Fig. 4.6a). The duration, spectral bandwidth and modal walk-off (Fig. 4.6c-e) all increase monotonically within the multimode, normal-dispersion gain fiber. The combined action of the spatiotemporal saturable absorber, and 

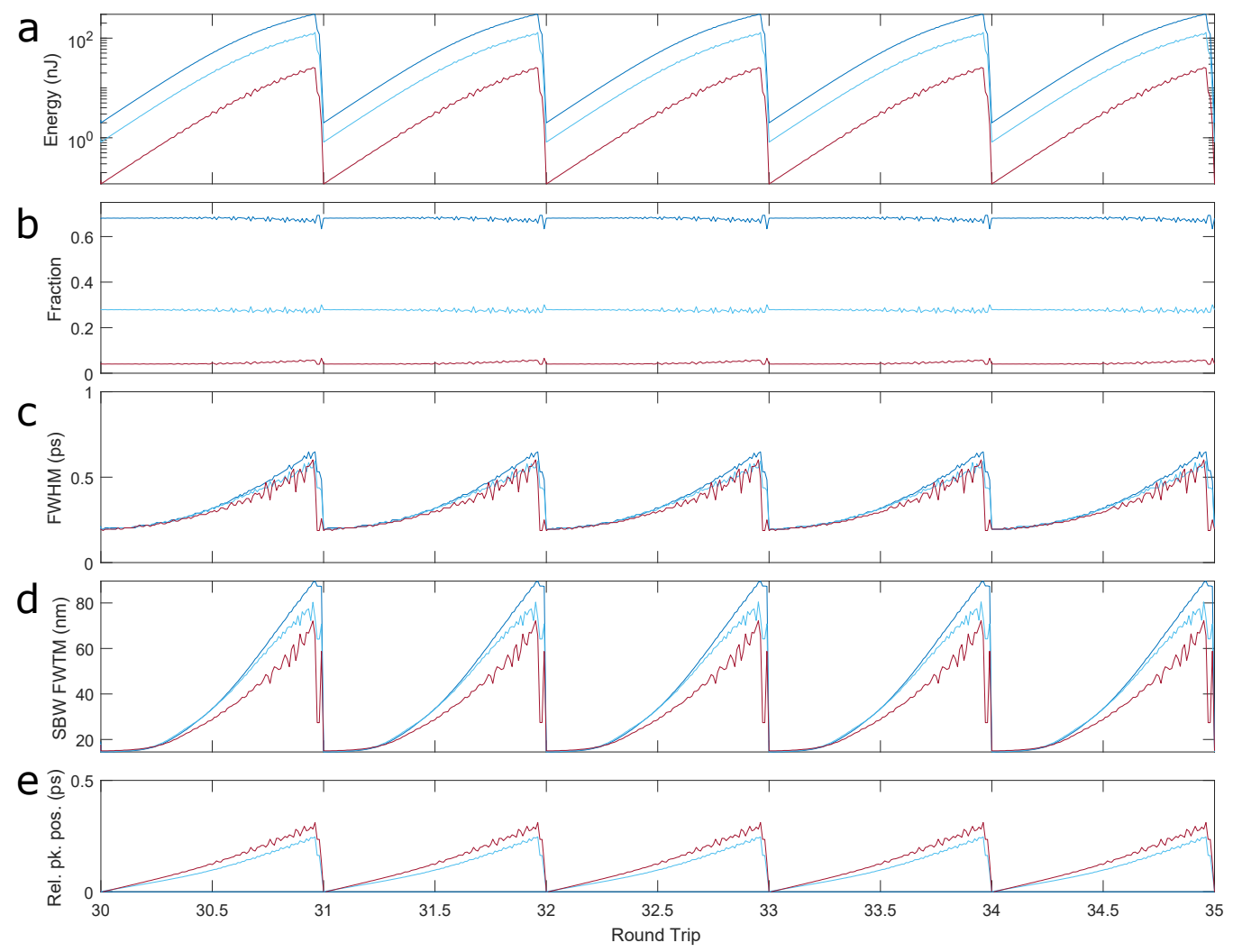

Figure 4.6: Intracavity evolution of the numerical STML example. The plot shows, for the mode-locked transverse mode families, the intracavity evolution of (a) the energy, (b) the fraction of the pulse energy in each mode (c) the full-width-at-half-maximum (FWHM) pulse duration, (d) the spectral bandwith (measured at full-width at 10\% maximum, FWTM), and (e) the temporal position of maximum intensity in each mode, relative to the peak intensity in the fundamental mode (i.e., the modal walk-off).

the spatial and spectral filter, act to restore all three parameters to their initial values. Thus, to a first approximation the evolution can be viewed as a 3D generalization of normal-dispersion mode-locking in $1 \mathrm{D}[39,72]$, where a narrow spectral bandpass filter is employed to shorten the duration of a linearly chirped pulse once per round-trip. It is, of course, worth emphasizing that this is almost certainly not the only way STML may occur: several others will be discussed briefly, and many more are likely waiting to be explored. Nonethe- 
less, this example is a useful starting point, with many features that appear to be common across different types of STML.

One of the most important concepts illustrated by the previous figures is why modal dispersion matters. In lasers with large modal dispersion, it is clear that the pulse in each mode would separate in time. While a suitable spatial filter could force the laser to operate in multiple transverse modes, pulses formed in each mode would not efficiently interact with one another, and would not enhance each other's transmission through the saturable absorber (whether a spatiotemporal one or not). This situation is precisely that of every previous attempt to realize a laser based on MM fiber[68]. In these lasers high-power pulses were severely unstable to breaking into multiple, disconnected pulses in different modes.

\subsection{First experimental realization}

Consideration of the complete problem defined by Eqns. 4.8 to 4.11 is considerably complicated by the structure of the slowly saturating gain term. Moreover, experiments on mode-locked multimode fiber lasers require relatively high power levels, high enough that it is very easy for a mistake to result in the costly destruction of fiber or other components. Finally, obtaining a multimode, rare-earth-doped fiber exhibiting low modal dispersion is not easy. Thus, if we want to build a laser with the lowest modal dispersion possible, we need to begin with a passive MM fiber. To do this, we built a simplified cavity in which the gain fiber was a single-mode fiber. In this case, the gain fiber acts as a spatial filter, and the gain physics is significantly simplified, since within 


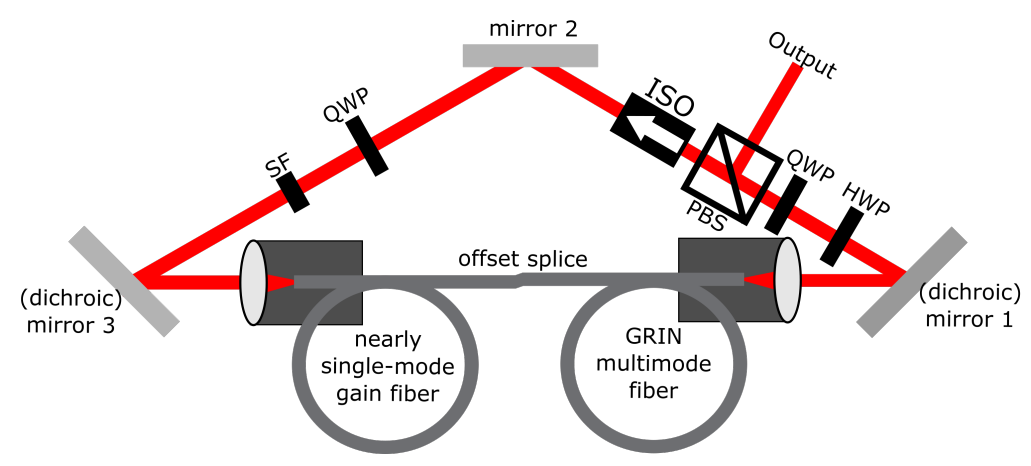

Figure 4.7: Schematic of the first, simplified MM laser cavity. Nonlinear polarization rotation is used as an effective saturable absorber in a ring cavity, requiring a polarizing beamsplitter (PBS) and several half-wave (HWP) and quarterwave plates (QWP). The gain fiber is, for all practical purposes, single-moded, and is spliced to a multimode passive GRIN fiber with an offset at the center to excite many modes. A spectral filter (SF) plays a role in the pulse evolution, while the isolator (ISO) ensures unidirectional lasing. Dichroic mirrors are used to couple a 976-nm pump into and out of the fiber.

the gain fiber only a single local mode is present. As later sections will expand on, even with this simplification there are notable approximations being made with this model. However, with this simplification, the approximations left are ones that are ubiquitous in the modeling of ultrashort-pulsed femtosecond fiber lasers[39, 72].

Naturally, if a spatial filter is appropriately chosen, one expects only the fundamental transverse mode of the cavity to lase, or another transverse mode, regardless of how many modes are supported in the gain fiber or in other fibers within the cavity. However, for the vast majority of spatial filters one might use, the highest transmission through the filter will be a superposition of transverse modes. Similarly, the 3D pulse which minimizes the loss through the saturable absorber will not in general be a one which resides exclusively in a single transverse mode.

To make sure that, indeed, our simplified cavity operated in a multi- 

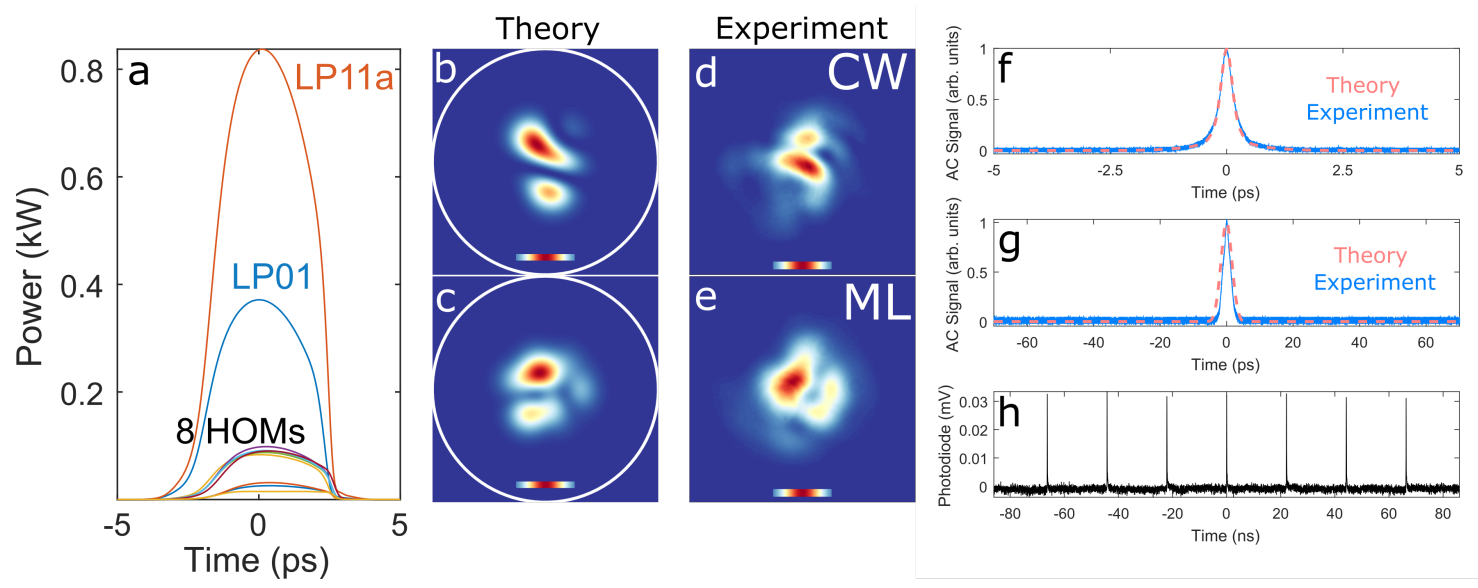

Figure 4.8: Experimental and theoretical results in the first cavity. (a) shows the theoretical steady-state mode-locked pulse, resolved into the modes. (b) shows the beam profile expected for continuous-wave lasing at maximum efficiency, and (d) shows the experimental measurement. (c) shows the expected beam profile in the mode-locked state, while (e) shows the experimental measurement. (f) shows the intensity autocorrelation of the pulse after dechirping using a pair of diffraction gratings, while $(\mathrm{g})$ shows the chirped pulse, without external dispersion compensation, on a long-range. (h) shows the pulse train, measured using a fast photodiode and oscilloscope with $<50 \mathrm{ps}$ resolution. The scale bars in the beam profiles are a 1D plot of the Gaussian profile of the fundamental mode of the waveguide. For the theoretical beam profiles, the fiber core is plotted in white; the experimental measurements are plotted on the same scale.

transverse mode regime, we spliced passive MM GRIN fiber to the gain fiber with a large offset between the fiber centers (Fig. 4.7). This ensured that many transverse modes of the MM fiber were excited. The laser was aligned to maximize the continuous wave power below the mode-locking threshold. In continuous wave operation, intermodal nonlinear interactions can be neglected, and the fibers were short enough $(<10 \mathrm{~m})$ such that disorder can be considered small. Thus, the output beam profile of the laser showed to a good approximation the transverse modes which were excited in the fiber. Fig. 4.8 shows this continuous wave beam profile (in experiment and simulation respectively) in an example cavity, chosen for a moderate offset for which roughly 10 transverse modes are 
excited.

When the pump power is increased above a certain threshold, the laser field suddenly transforms. This transformation occurs in time as well as space, it is spatiotemporal. That is, the beam profile changes abruptly as the field self-organizes due to nonlinear interactions between transverse modes, timedomain measurements show the formation of a femtosecond-scale pulse emitted once per round-trip, and and spectral measurements show a broad spectrum, typically with steep edges characteristic of normal-dispersion modelocking [47]. All these changes are of course coupled together, as the forming pulse is spatiotemporal, spatiotemporally mode-locked. In my opinion, this spatiotemporal phase transition is the strongest (and certainly the most exciting) evidence that the laser is spatiotemporally mode-locking.

More detailed measurements of the mode-locked state confirm that the laser field consists of multiple transverse modes, which together comprise a single, coherent multimode pulse. Long-range autocorrelation (Fig. 4.8f-g) shows the short duration of the pulse and, when combined with fast photodiode measurements of the pulse train, forces us to conclude that the pulse is alone in the cavity (that is, there is no background of continuous wave lasing, or multiple sub-pulses). Thus, we have to conclude that the multimode beam profile belongs to the multimode, spatiotemporally mode-locked pulse.

The pulse evolution of this laser, according to simulations, is quite similar to the one shown earlier in this chapter for a laser with a spatial filter and a MM gain medium. That is, spectral filtering, spatial filtering, and spatiotemporal saturable absorption all act to counteract the pulse broadening caused by modal and chromatic dispersion. Different transverse modes become phase- 
locked through nonlinear interactions as they form a coherent pulse that minimizes the loss (or maximizes the gain) for the field through the cavity.

Now, as you might expect, there is a lot more that can be seen with this laser, and an overwhelming number of STML states have been observed, including multi-periodic states, as well as ones comprising roughly 100 transverse spatial modes. More details on some of these are given in Ref. [73]'s supplementary material.

\subsection{Fully-multimode fiber cavity}

While the simplified cavity does manage to exhibit most of the physics of STML, it is ultimately a bit contrived to have a single-mode fiber (or nearly single-mode fiber) in the cavity. From the point of view of really exploring STML, and of realizing high-power, flexible ultrafast fiber lasers, we need to be able to also observe STML in a laser with a MM gain medium.

\subsubsection{Justifying the model}

In such a laser, we need to be a little more careful about how our model works. Let us examine in a little more detail where the model, Eqns. 4.8 to 4.11 , comes from, as well as its deficiencies. The gain introduces a second timescale in addition to the fast one of the Kerr nonlinearity.

The first timescale is the timescale of fast nonlinear interactions, principally through the Kerr nonlinearity and the saturable absorber. In our investigations 
so far, the saturable absorber is always a result of the Kerr nonlinearity. For a MM field, the amount of light that is coupled out of the cavity from the polarizing beam splitter depends on the accumulated nonlinear phase shift at each point in space-time. The loss of this nonlinearity-dependent output coupling, as seen by the circulating pulse, is a spatiotemporal saturable absorber. It is fast in the sense that it depends on the nonlinear phase shift at every instantaneous point in spacetime within the pulse, but however the loss is only manifested once per round trip. Thus, the model laid out earlier does not precisely describe this, but it is qualitatively correct.

The second timescale, which is significantly more relevant for a laser in which the gain medium is multimode, is that of the gain dynamics. Gain saturation, as it is modeled in Eqn. 4.8, results because the gain medium has only a finite number of excited ions for a given pump power. In a single-mode gain medium, all that matters is the number of excited ions in the core. However, in a multimode waveguide, different modes overlap with different regions of the core. The different modes also overlap with one another to different extents. The result is that we have to consider the depletion of excited ions throughout the cross-section of the fiber.

In our model, the gain saturation in $(x, y)$ occurs in proportion to the integral of the pulse across the entire time window. This is an approximation. First, we are supposing that the gain dynamics (decay of the population or relaxation oscillations) are very slow relative to the pulse dynamics. This is true to a good approximation in $\mathrm{Yb}$ fiber lasers. That said, we are in effect saying that the gain saturation occurs within the longest timescale of the simulation. For the sake of computer memory, we typically do not consider the entire round trip time in 
simulation, but instead consider just the 100 ps or so around the pulse. In fact, the amount of energy that can be stored in excited ions within a typical doped fiber is much larger than the pulse energy of even an exceptionally high-power mode-locked fiber laser, by a factor of at least 1000 . Thus, to really model gain saturation correctly we would need to extend our integration bounds to include the last 1000 simulated round trips (at which point the gain dynamic timescales would indeed be relevant). This is not practical. Even with optimized codes and using a subset of the fiber modes, simulating the pulse propagation across a 2$10 \mathrm{~m}$ long fiber cavity takes at least 1 hour. Thus, to simulate the spatiotemporal mode-locking transition, where a multimode pulse forms from fluctuations of the continuous wave field, then stabilizes into a steady-state evolution, would take several months. This approximation has fortunately not prevented very accurate modeling of single-mode ultrafast fiber lasers, where it is almost ubiquitously employed. This should comfort us a little when it comes to STML, and so far we have observed rather remarkably good qualitative agreement between this model and experimental observations. However, because MM lasers can exhibit much more complex dynamics than SM lasers, it is nonetheless important to remember these inherent approximations.

\subsubsection{Experimental and numerical results in cavities with mul- timode gain media}

The first numerical example considered in this chapter was a laser in which the gain medium was multimode (in the example, 7 modes were considered). This

is one typical type of STML state observed. Our initial experimental measure- 
ments of this kind of state are reported in the supplementary material of Ref. [73].

Although while I am writing this thesis, our investigations are still ongoing, it is worth introducing two other typical STML states, the 'slow-fast dissipative soliton', and multi-periodic STML states. We will examine both numerical results as well as experiments. For the experiments, the cavity design is virtually identical to the one depicted in Fig. 4.7, except that the fibers are replaced by a single, multimode gain fiber.

\section{Slow-fast dissipative solitons}

Another important type of STML state is the slow-fast dissipative soliton, which results from the interaction of strong saturable absorption, that is fast nonlinear losses, with slow gain competition. Much like the first state considered, this type of STML is characterized by a spatiotemporal phase transition, where the field self-organized in 3D to form a steady-state pulse that is constant from round trip to round trip. The key difference, however, is that even though many transverse modes may be involved in the continuous wave lasing state observed at incrementally-lower pump power, at steady-state the slow-fast dissipative soliton occupies a single transverse mode (or a family of degenerate transverse modes).

Figure 4.9 shows a simulation of this type of mode-locking. The reason it is called a 'slow-fast dissipative soliton' is that, despite the laser nominally supporting multiple transverse modes, it selects a single one through a combination of (fast) nonlinear loss, whereby modes with low amplitude are attenuated 
each round trip in the spatiotemporal saturable absorber, and (slow) exponential gain competition, wherein the most energetic mode extracts the majority of the energy available in the gain medium. These two processes, one being an ultrafast nonlinear interaction between all the 3D modes and the other being a slow interaction in the spatial domain only, create and sustain the spatiotemporal self-organization of the slow-fast dissipative soliton.
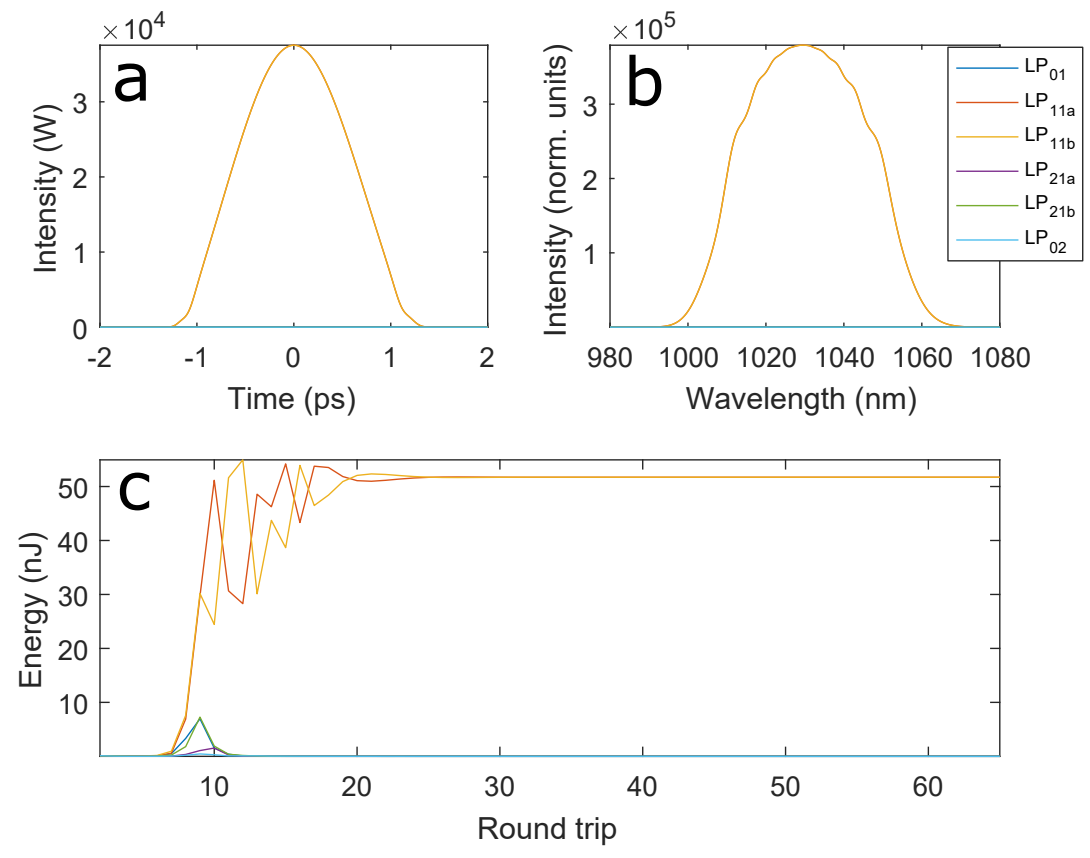

Figure 4.9: Slow-fast dissipative soliton, numerical example. A slow-fast dissipative soliton is a stable, mode-locked pulse that forms in a single mode or group of degenerate modes due to the combined effects of (slow) exponential gain competition and (fast) spatiotemporal saturable loss. Here, the pulse contains identical quantities of two LP11 modes. (a) shows the steady-state, moderesolved pulse, while (b) shows the steady-state, mode-resolved spectrum. (c) shows the formation of the SFDS from the initial noise launched into the cavity.

Several features of the SFDS are worth remarking on. First, the SFDS is a robust form of STML which may emerge even in lasers with quite strong modal dispersion. This is practically useful, since most MM fibers have strong modal 
dispersion, especially those exhibiting environmental stability. To avoid the fate of earlier MM fiber mode-locked laser attempts, this requires that very strong saturable absorption be employed. At time of writing, I am optimistic that this may be realized by the Mamyshev mechanism [74, 75, 76] we have recently explored as an effective saturable absorber in SM lasers. Second, the SFDS terminology refers to the space-domain. In the time-domain, the SFDS may exhibit an evolution similar to the amplifier similariton [72], dissipative soliton [39], as well as intermediates or other kinds of evolutions. While some coupling between space and time is critical, especially in the formation of the SFDS from noise, no particular constraint is placed on the steady-state time-domain evolution aside from strong saturable absorption and gain. Finally, while the purity of the mode is perhaps less exciting scientifically than other types of STML, for many modern applications this is beneficial, especially when the selected mode is the fundamental, Gaussian mode that most modern laser applications expect.

Rigorously confirming the SFDS in experiments will require the development of better techniques for measuring the modal composition and 3D shape of STML pulses, and most likely the reliable high-contrast saturable absorption of Mamyshev-type saturable absorbers. The former is discussed in the final chapter. However, it is likely that STML states close to, if not precisely, SFDS operation have been in observed in the laboratory. The final figure of Ref. [73] shows one probable candidate, in which the SFDS pulse appears to comprise a combination of LP21 modes. 


\section{Multi-periodic evolutions, and more}

Another important class of STML states are those in which a coherent MM pulse forms, but the pulse is not precisely the same each round trip. Rather, multiple round trips are required before the same pulse is observed. Before examining these in more detail, a few contextual remarks. First, this is the the first kind of 3D mode-locked pulse ever conceived of, and for a long time was the only kind of 3D mode-locked pulse ever conceived of. Auston [77] and Smith [78, 79] considered mode-locking involving multiple transverse and longitudinal modes. They considered gas-based lasers with resonators comprised of two mirrors, so that the resonant frequencies are:

$$
\omega_{m n q}=\frac{q \pi c}{d}+\frac{c}{d}(m+n+1) \cos ^{-1}\left(1-\frac{d}{R}\right)
$$

where $q$ is the longitudinal mode index, $m$ and $n$ are the indices of the Hermite-Gaussian tranvserse mode, $d$ is the resonator length and $R$ are the radii of the mirrors. In the limit as $d>>R$, the transverse mode spacing is equal to the longitudinal mode spacing. In this limit, Auston and Smith saw the possibility for 3D, or 'total', mode-locking in which a pulsed beam was formed, which would oscillate in the transverse plane at a rate determined by the the transverse mode spacing, and in the longitudinal direction by the longitudinal mode spacing.

Given our earlier discussion, it should be obvious now that the condition that the longitudinal and transverse mode spacings are the same is not necessary. In a fiber laser the transverse mode spacing is much smaller than the longitudinal mode spacing. As previous examples show, nonlinear and lin- 
ear coupling between the modes can in this case cause a STML pulse which is characterized by a simple frequency comb spectrum, with the same pulse being emitted at a repetition rate near the cavity fundamental repetition rate. However, there is no reason why this may be the only stable outcome. Especially as the pulse contains a significant variety of modes, STML may equally manifest as a pulse whose intermodal phases, modal energy distribution, or other quantities, repeat over multiple, rather than a single, round trip. The spectrum in this case exhibits long-range order, but must have a frequency unit cell larger than conventional mode-locking and the previously-described STML states. A very simple example is shown in Fig. 4.3b.

We observe these kind of STML states in both laser cavity designs described so far, although more commonly in cavities comprised of only MM gain fiber. These multi-periodic states are also observed in simulations, a fact that is somewhat surprising given our mistreatment of the gain saturation timescale.

Fully exploring or describing this category of STML would go quite beyond the intended level of detail of this thesis. States in this category include many different periods, as well as possibly chaotic or aperiodic sequences. The pulse may exhibit oscillating intermodal phases, amplitudes, or both, and through these, other parameters of the pulse may oscillate from round-trip to roundtrip. In simulations and experiments alike, they are most commonly observed by introducing perturbations to STML states similar to the previous examples. Thus, they may reflect the adaption of a given STML pulse to a changing environmental parameter, but perhaps not the global optimal pulse that would be obtained from an initial noise condition.

Figure 4.10 shows the simulated output of a MM laser, where pump power 
(roughly related to the gain saturation fluence) is slowly increased. This illustrates a variety of different types of STML. Similar sequences of many different types of STML may be observed, for example, by varying the spatial filter position or dimensions [73].

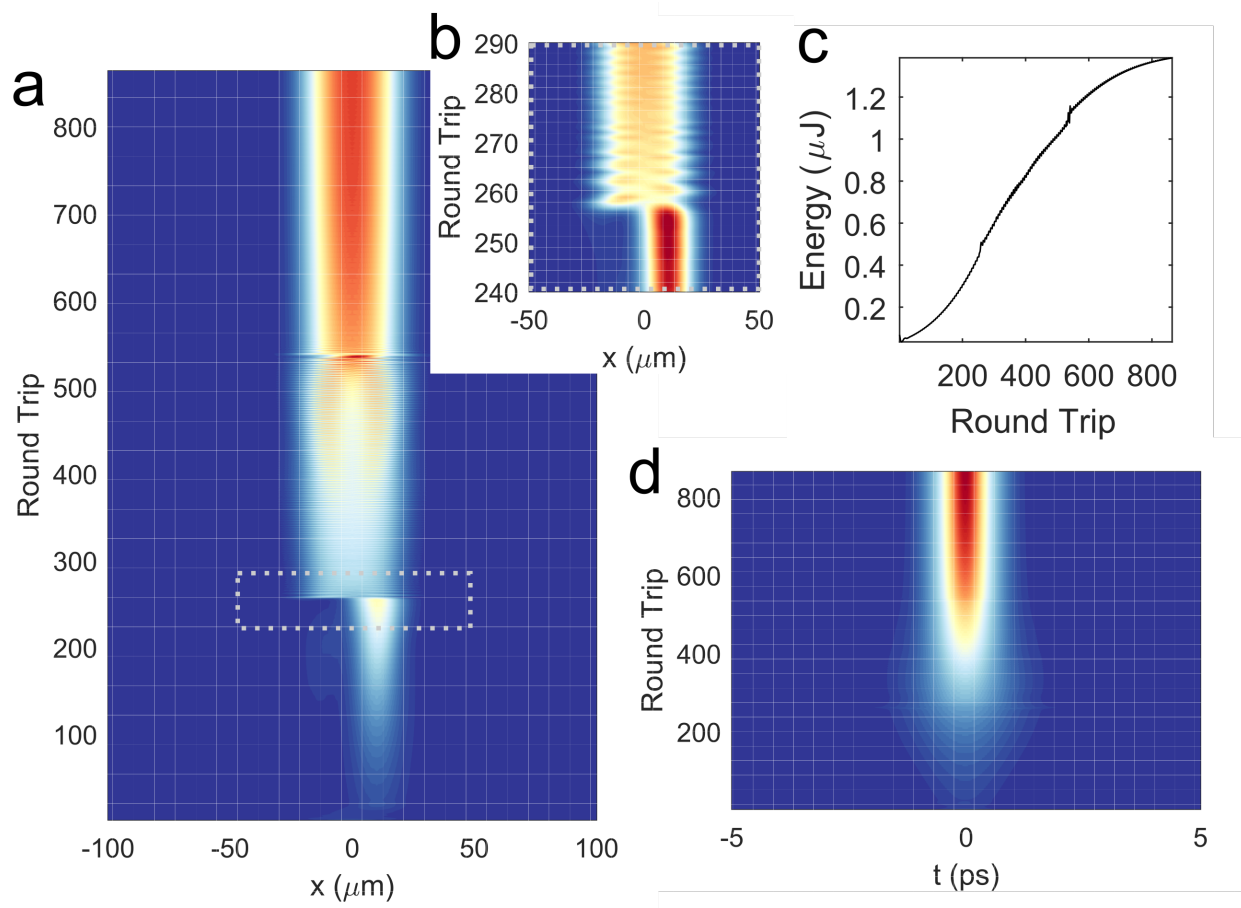

Figure 4.10: Example of different types of STML states observable by slowly changing one parameter of the system. (a) shows the spatial intensity profile (in 1D) of the simulated pulse. At low power (early round trips), the pulse exhibits the same MM beam profile and pulse parameters from round-trip to round-trip. At higher-powers, the pulse transitions (zoom in (b))to a spatially-oscillating multi-periodic state whose period changes with increasing pump power. Finally, at the highest-power, a stable Gaussian beam emerges. (c) shows the pulse energy output from the cavity, while (d) shows the pulse intensity in time.

Currently, exploring the full space of STML in experiments is challenging due to technical issues such as the damage of fiber end facets by the high-power pulses. These issues will easily be solved in time, and we can still glimpse experimentally some of the richness seen in simulations. Fig. 4.11 shows a corresponding experiment, where the pump power is increased and different types 
of STML are observed. At low power (Fig. $4.11 \mathrm{~g}$ and $\mathrm{h}$ ), the laser emits only continuous wave. At higher pump power, Fig. 4.11e-f, the field transitions to spatiotemporal mode-locking. As the pump power is increased further, the autocorrelation (Fig. 4.11k) shows the pulse transition from a multi-peaked shape to a single peak (bottom to middle curves). At higher pump powers still, the beam profile exhibits a significant change, while the autocorrelation remains a single pulse (Fig. 4.11a-b, and top row of Fig. 4.11k). Fig. 4.11i shows the temporal intensity of the field, as measured by a fast photodiode, corresponding to the four states shown on the left-most plots. The intensity intially exhibits longitudinal mode-beating, then a stable pulse train and finally the emergence of a multi-periodic state. Fig. 4.11j shows the latter state's RF spectrum. It is worth remarking the extremely high contrast of the RF spectrum evidences that even as the STML state exhibits oscillations, it remains very temporally coherent. 

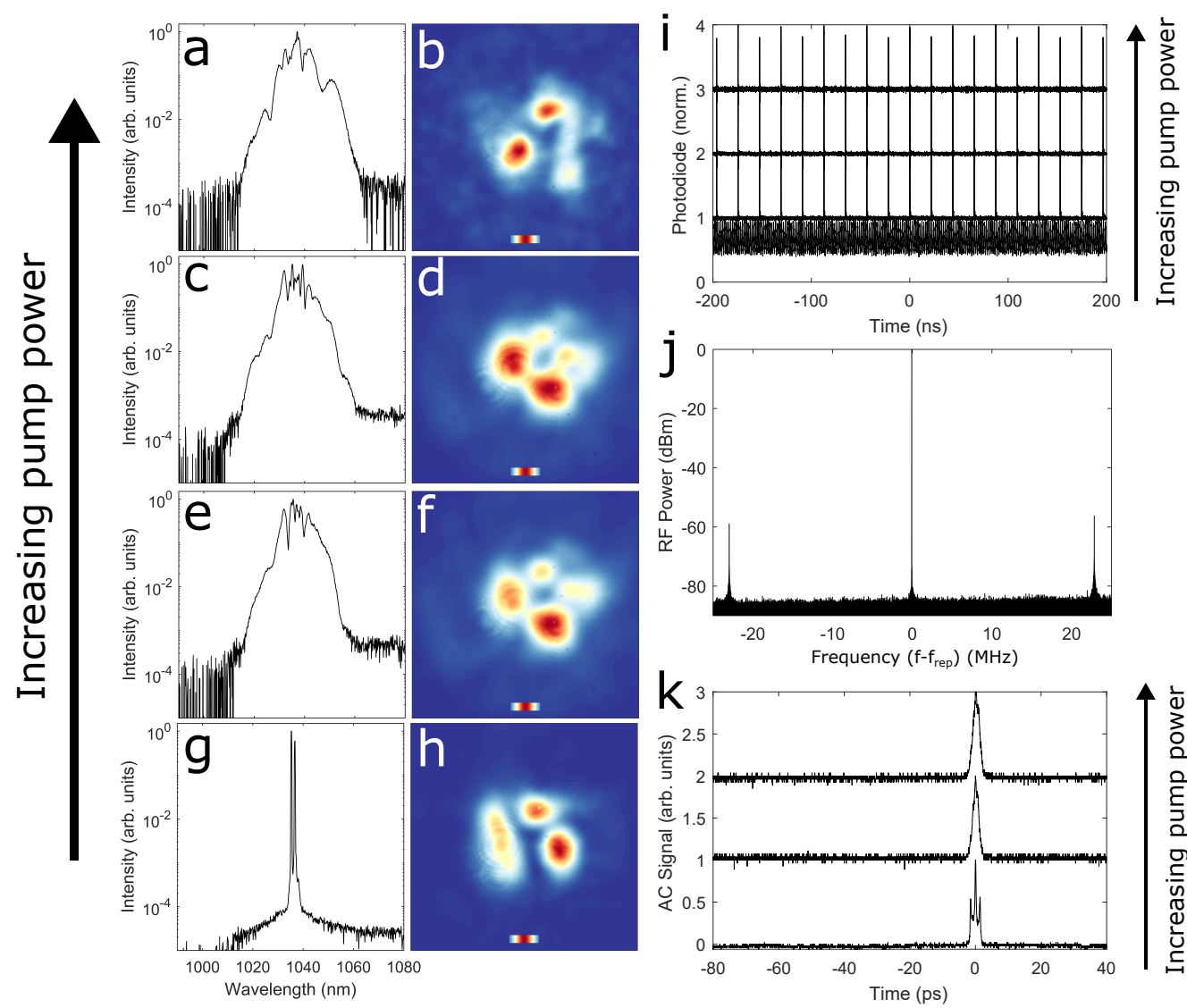

Figure 4.11: Experimental example of different types of STML states observable by slowly changing one parameter of the system. The beam profiles and spectra (a-h) correspond to increasing pump power. (i) shows the fast-photodiode signal of each of the 4 states shown, with the lowest CW longitudinal modebeating, and the highest a multi-periodic STML state. (j) shows the RF spectrum of the highest-power STML state shown. The high contrast and narrow spectral sidebands at $\pm f_{\text {rep }} / 2$ show the coherence of this state. $(\mathrm{k})$ shows the long-range intensity autocorrelations of the mode-locked states. Combined with (i), these also confirm that only 1 pulse is present in the cavity (except in the lowestpower mode-locked state, where two distinct peaks are observable). 


\section{CHAPTER 5}

\section{FUTURE DIRECTIONS}

Finally, we arrive at what might be the most exciting chapter. Here, I will briefly overview a selection of paths forward for the study of MMNLFO. As of 2018, the public disclosure of patentable ideas makes pursuing a patent impossible. Whether or not one subscribes personally to the 4th scientific ideology of 'economically-motivated science/technology', as a scientist you ought to take it seriously as a societal force. In modern society patents play such a crucial role in the mainstream development of new technologies that they are often prerequisites for successful commercialization. Successful commercialization is almost always a prerequisite for the wide adoption of physical tools (or almost any technology), like those we might eventually create with ultrafast MM lasers or related light sources. Refs. [1] and [2] include plenty of discussion of future directions for MMNLFO, with the latter detailing specific routes to flexible, environmentally-stable and ultrahigh power fiber sources based on MM fiber.

In view of these things, in this chapter I will focus on the science of MMNLFO, and specifically on fertile directions for the work. This 'direction' focus lacks in specific, concrete proposals, but I hope it will excite some reader's imaginations and creativity to suggest their own.

The directions:

1. Better experimental measurements and methods: build more capable, mode-resolved and 3D measurement techniques, and tools to directly interface with modes in the lab.

\section{Highly multimode nonlinear waves with 'multi-media' effective waveg-}


uides: explore high-power spatiotemporal nonlinear waves in multi-layer media, and in passive and active free-space multimode resonators.

3. Complex nonlinear optics: exploit machine learning to optimize linear, nonlinear and quantum-optical multimode effects, and to assist and control STML.

4. Spatiotemporal nonlinear optical 'walks' and light control: use the controllability of the propagation of high-power multimode fields to rapidly and precisely control the propagation of other classical and quantum light fields

\subsection{Advances in MMNLFO experimental methods}

Better experimental techniques are at the top of the list because they will open up much more of the world of MMNLFO, and enable much more precise applications and understanding. The basic goal of these techniques is to understand the 3D, spatiotemporal structure of multimode light, and to allow that structure to be controlled, mode-by-mode.

The main challenge to this end has been that the default approach, using spatiotemporal pulse measurement techniques, faces harsh limits in terms of the total dimensions of pulses that can be measured, or requires such lengthy scanning sequences that they are rarely useful for routine measurements. As the previous chapters have shown, MM fields, even those consisting of just a few modes, can often be extremely complex in space-time, extending over relatively large time-bandwidth and space-bandwidth products. In contrast, it seems much more promising to exploit the efficient compactness of the mode 
basis.

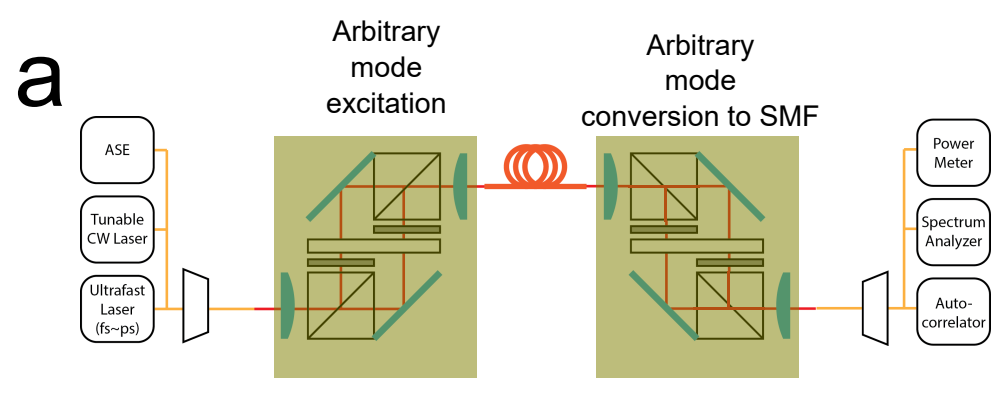

b

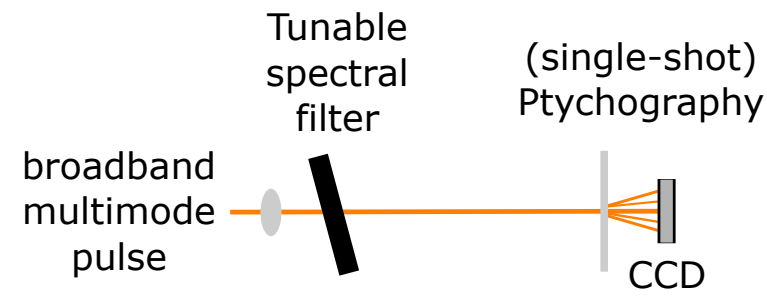

C

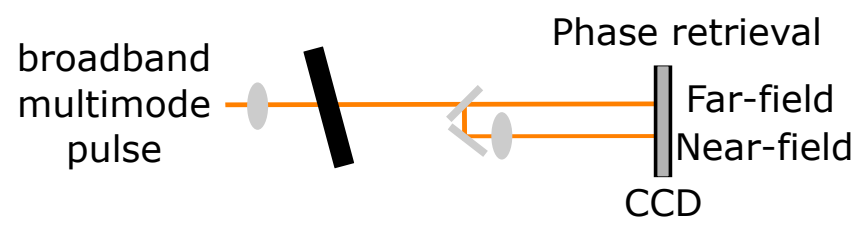

Figure 5.1: New approaches for better measurement and control of MMNLFO experiments. (a) Using SLMs as adjustable phase-plates allows conversion to and from a single-mode Gaussian beam into arbitrary coherent combinations of fiber modes. On the input of the fiber, this allows precise excitation and controlled experiments. On the output, this allows full characterization of the field using techniques developed for single-mode Gaussian beams. (b-c) Using phase-retrieval or ptychographic inspired approaches, the complex mode amplitudes may be retrieved from intensity measurements of a multimode field at a given wavelength. By repeating this procedure for multiple wavelengths, broadband multimode fields may be measured.

Besides just being more efficient and faster, physically resolving a MM field into a small number of modes (Fig. 5.1a) can also allow for all the tools and techniques applied to the measurement of single-spatial-mode fields, such as autocorrelation, electric-field measurements like FROG, routine simple measure- 
ments like the spectrum, or other techniques that allow deeper insight, like dispersive Fourier transformations for single-shot spectra[80]. Physically resolving the field into its modes also allows for direct manipulation of the field in each mode. This will be useful for applications as well as measurements. A flexible approach uses spatial light modulators to apply the correct phase to transform the desired mode combination into a Gaussian matched to a subsequent single-mode fiber[81]. However, especially for high-power applications other approaches, like multi-plane light conversion[82], will be worth exploration.

A second approach to mode-resolved measurements is modal phaseretrieval, using algorithms to computationally decompose intensity measurements into the modal composition (Fig. 5.1b-c)[83, 84, 85, 86]. In the case of narrowband MM light, these approaches may be applied straightforwardly. For broadband MM light, this may be applied on a wavelength-by-wavelength basis, thus allowing a simple system to measure the mode-resolved spectrum of a complex broadband light field. The simplicity of this approach means that it can be relatively widely and cheaply applied, across in principle very broadband fields with whatever required resolution. It is not clear how this approach could be used for mode-resolved electric field measurement (that is, to retrieve $A_{m}(t)$ for each mode instead of just $\left|\tilde{A}_{m}(\omega)\right|^{2}$, but I am optimistic that it can be done (although probably with some constraints on the complexity or certain details of the pulse). This would be an extremely useful and flexible optical measurement technique, especially if it could be generalized to many modes, or even to full-field measurement.

While they have some short-term disadvantages due to not exploiting the mode basis, full-field pulse measurement techniques are still worth application 
and refinement, since they could ultimately have broad use within optics. In this regard, MMNLFO may serve as a testing grounds, or initial impetus, for application and development of full-field techniques like STRIPED-FISH[87] or SEA-TADPOLE[88].

\subsection{Highly multimode nonlinear waves in multi-pass cells, passive resonators and multi-layer media}

'Multi-media' waveguides and nonlinear optical environments represent an exciting means of realizing the entire world of MMNLFO (and beyond, or at least into its hyperspace). For brevity among other reasons, this section is unlikely to put specific, concrete uses of this approach into your head, but I hope you at least see the creative freedom and begin concocting some of your own.

Various realizations of nonlinear pulse propagation in multi-pass cells, pas-

sive resonators and multi-layer media[89, 90] are shown in Figure 5.2. Light propagation in the cell consists of diffraction within the free-space or bulk medium section, where the majority of the nonlinearity occurs. The mirrors or lenses provide an effective waveguiding potential, and the former may also be designed to provide dispersion control through engineering the dielectric coating. In such, 'hollow', or 'bulk waveguides', the optical intensity can be very high, with the peak power possibly exceeding the critical power for collapse. The properties of the 'waveguide' may be easily adjusted by replacing the mirrors, by designing more complex cells involving multiple different mirrors or media, by placing windows of different types of media in the cell, filling the cell with gas or liquid, or simply just changing the distance between the mirrors 
or lenses. While MMFs provide many useful features and will typically have much lower loss, it is hard to compare the flexible control over the 'waveguide' that one has with a multi-pass cell containing multi-layered, bulk or gas media. In addition, these cells may support physics that is very close to single-mode, or that involves many more modes than could ever be nonlinearly coupled in solid multimode fibers.
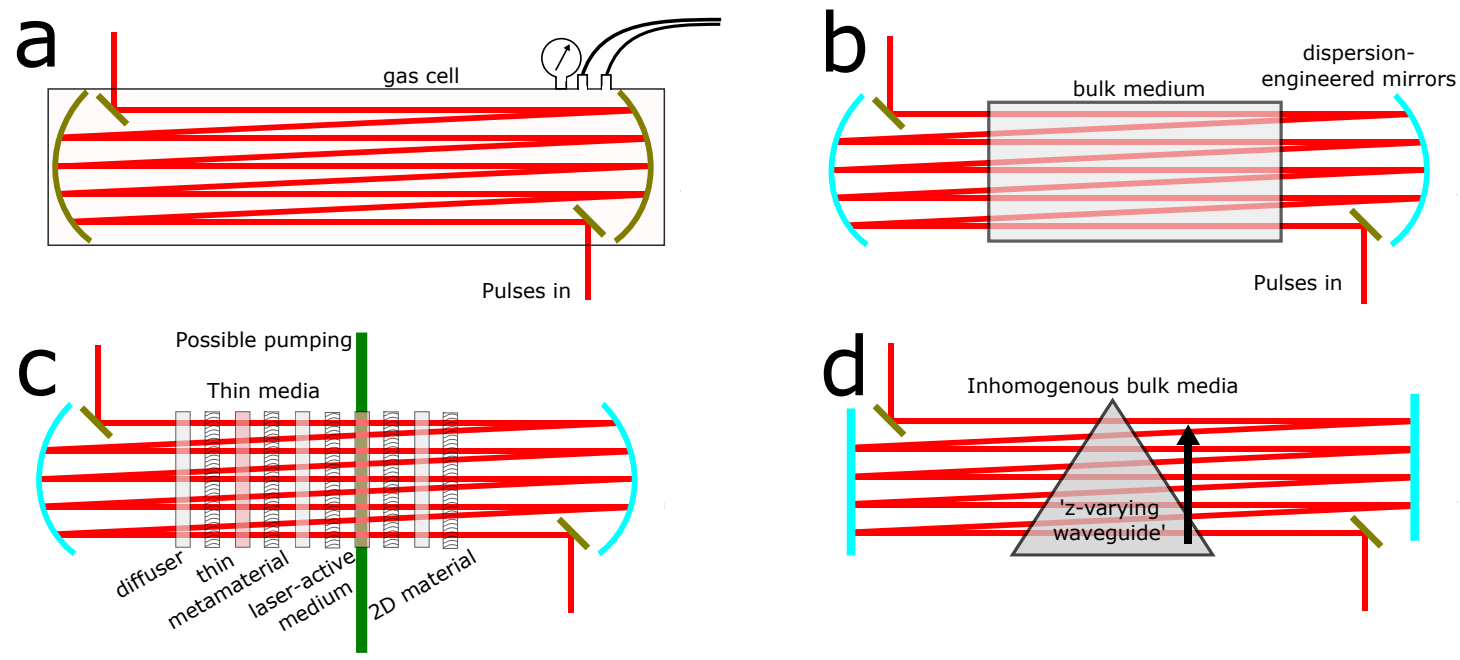

Figure 5.2: Multi-pass cells and resonators as flexible platforms for extreme nonlinear light sources and spatiotemporal nonlinear optical pulse propagation. The examples show different degrees of freedom for engineering and experimentation, but are not intended to show any particular specific application.

From an application perspective, multi-pass cells and bulk 'macroresonators' are promising for generating novel light sources, including compact sources of extreme light or non-optical laser-like light. While I can envision a handful of compelling, near-term applications with broad appeal, I am nonetheless more excited about what may be creatively devised for more extreme or 'exotic' goals. In some sense, this platform allows one to combine different materials or components into an 'effective' medium or waveguide, and direct nonlinear transformations of an incident field with few boundaries. This may 
be partly compared to a metamaterial, where the internal structure of a material may be manipulated so that its bulk properties may be very unusual. However, the multi-pass cell is, as a controllable nonlinear optical medium, quite a bit more flexible (with, to be sure, potentially similar limitations). Thus, recalling the first chapter of this thesis, I hope to eventually see the flexibility of 'multimedia' allowing for creative, cost-efficient laser sources of ultrahigh power or (ultra)-ultrashort duration pulses, bright coherent x-rays, THz or long-wave infrared light, perhaps even gamma radiation or particle beams.

\subsection{Complex nonlinear optics}

Had I been more successful with my PhD research, this thesis could have perhaps been titled 'Complex nonlinear optics'. This concept refers to two complementary directions. The first is the application of complex network descriptions to describing complex multimode pulse propagation. A glimpse of this and what it might accomplish is provided in Chapter 3 and Ref. [57]. The second is the use of MM nonlinear waveguides or nonlinear MM optical oscillators (and related media where complex nonlinear pulse propagation may occur) as 'nonlinearity reservoirs', through which to create whatever light one desires by control of the exciting field.

The final sections of Chapter 2 laid out the many different nonlinear processes which may be controllably excited with a single pulse at 1550-nm, in a short GRIN MMF with several hundred modes. A natural first step toward complex nonlinear optics would be to repeat this experiment with much richer

control over the initial field, likely just in space using wavefront shaping with a 
spatial light modulator, but potentially also in time and frequency using a pulse shaper ${ }^{1}$. Combined with controllable spectral filtering, or even a second spatiotemporal pulse shaper, at the fiber output, such a system would seem to be able to provide high-energy ultrashort pulses at virtually any wavelength desired, as well as perhaps more intricate outputs, such as sequences of pulses with different wavelengths, in different modes, or pulses with complex spatiotemporal structure. A key benefit of this technique is that it is generative rather than merely relying on linear modulation or dissipation of an initial broadband pulse. In this sense it is complementary to existing pulse shaping.

While such approaches seem to be doomed to never be fully-functional arbitrary spatiotemporal optical waveform generators, it is a fascinating question to understand their limits. A first guess is that this approach will be limited by the initial number of useful degrees of freedom one has. In this regard the nonlinearity of the propagation may help (or hurt) since it could make the output acutely sensitive to the input controls. This could be useful in extracting the maximum effect from the precision of the input degrees of freedom. However, more likely this is something that would need to be managed carefully. A reasonable strategy for practical devices would be, instead of attempting completely arbitrary field generation, to produce a source with a pre-calibrated range of outputs. In this case, a user would instead be able to choose from a long list of options, and designers would attempt to include in the list all the options that would be conceivably be relevant to users.

On the other hand, this eliminates the possibility of really creating tailored fields precisely for applications. As was alluded to in the chapter on MM lasers,

\footnotetext{
${ }^{1}$ To some extent, this is already being done in the literature, by Tzang et al.[91] as well as in some spirit by Florentin et al.[92].
} 
MM lasers or optical oscillators generally, can be thought of as high-dimensional field optimizers, with incredibly many continuous degrees of freedom. Thus, in my opinion a more powerful and exciting long-term approach will be to, instead of simply relying on the nonlinear complex medium in a single-pass, to attempt to use low-dimensional controls and feedback to direct the optimization of fields by the nonlinear MM oscillator itself. The right realization would be a marriage between computer-controlled wavefront and pulse shaping concepts with the self-optimizing nonlinear dynamics of the highly-multimode optical oscillator. A practical vision might still rely on a 'list' of preset configurations, within which the user would be able to provide a feedback for the system to perform a local optimization around this starting point. This could be especially exciting in the context of application-specific, real-time feedback: even in the likely situation that a user cannot specify the exact perfect field for their application, such a system would be able to find it automatically.

Obviously, a long term dream would be a device that could perform completely arbitrary field generation optimization, from whatever feedback a user provides. It would, in all senses of the word, be the ultimate light source, and perhaps the ultimate tool, being able to, in theory, find a field to accomplish any task for which a learning phase could be allowed, and the laws of physics were obeyed. 


\subsection{Controlling light propagation in 4D: spatiotemporal non- linear optical 'walks' and routing}

The concept of using nonlinear pulse propagation to control the 4-dimensional properties of the light leaving a multimode waveguide can be extended to controlling the 4-dimensional propagation of light within the multimode waveguide. By controlling a high-power pump field launched into a multimode waveguide one has the ability to control, through cross-phase modulation, the propagation of a second, weaker field at a different wavelength. The field $I(x, y, z, t)$ of the pump comprises a 4-dimensional index perturbation to the waveguide seen by this weaker field.

If we ignore the time dimension, this means that the pump field may be used to rapidly change the modal composition (phase and amplitude) of the weaker field, and in fact to perform arbitrary linear optical transformations (to some degree, nonlinear optical transformations may be possible; except perhaps with an oscillator, they will not however be arbitrary as discussed in the last section). This controllable optical transformation has a clear application in many

optical information processing applications, such as in optical neuromorphic computing or signal processing and routing for space-division multiplexing. In the quantum domain where the 'weak' field is formed of single photons, this setup would implement a controllable quantum walk among the spatial modes. The time dimension of the pump, however, means that this controllable routing/walking extends into spacetime. This could allow for optical or quantum optical information processing, based primarily on 'linear' quantum walks and transformations, involving light at many different frequencies as well as spatial 
modes. 


\section{BIBLIOGRAPHY}

[1] L. G. Wright, Z. M. Ziegler, P. M. Lushnikov, Z. Zhu, M. A. Eftekhar, D. N. Christodoulides, and F. W. Wise, "Multimode Nonlinear Fiber Optics: Massively Parallel Numerical Solver, Tutorial, and Outlook," IEEE Journal of Selected Topics in Quantum Electronics 24, 1-16 (2018).

[2] W. Fu, L. G. Wright, P. Sidorenko, S. Backus, and F. W. Wise, "Several new directions for ultrafast fiber lasers [Invited]," Optics Express NA, NA (2018).

[3] C. Xu and F. W. Wise, "Recent Advances in Fiber Lasers for Nonlinear Microscopy." Nature photonics 7, 875-882 (2013).

[4] D. A. Orringer, B. Pandian, Y. S. Niknafs, T. C. Hollon, J. Boyle, S. Lewis, M. Garrard, S. L. Hervey-Jumper, H. J. L. Garton, C. O. Maher, J. A. Heth, O. Sagher, D. A. Wilkinson, M. Snuderl, S. Venneti, S. H. Ramkissoon, K. A. McFadden, A. Fisher-Hubbard, A. P. Lieberman, T. D. Johnson, X. S. Xie, J. K. Trautman, C. W. Freudiger, and S. Camelo-Piragua, "Rapid intraoperative histology of unprocessed surgical specimens via fibre-laser-based stimulated Raman scattering microscopy," Nature Biomedical Engineering 1, 0027 (2017).

[5] K. Sugioka and Y. Cheng, "Ultrafast lasersreliable tools for advanced materials processing," Light: Science \& Applications 3, e149-e149 (2014).

[6] R. R. Gattass and E. Mazur, "Femtosecond laser micromachining in transparent materials," Nature Photonics 2, 219-225 (2008).

[7] R. L. Sandberg, A. Paul, D. A. Raymondson, S. Hädrich, D. M. Gaudiosi, J. Holtsnider, R. I. Tobey, O. Cohen, M. M. Murnane, H. C. Kapteyn, C. Song, J. Miao, Y. Liu, and F. Salmassi, "Lensless Diffractive Imaging Using Tabletop Coherent High-Harmonic Soft-X-Ray Beams," Physical Review Letters 99, 098103 (2007).

[8] T. Popmintchev, M.-C. Chen, D. Popmintchev, P. Arpin, S. Brown, S. Alisauskas, G. Andriukaitis, T. Balciunas, O. D. Mucke, A. Pugzlys, A. Baltuska, B. Shim, S. E. Schrauth, A. Gaeta, C. Hernandez-Garcia, L. Plaja, A. Becker, A. Jaron-Becker, M. M. Murnane, and H. C. Kapteyn, "Bright Coherent Ultrahigh Harmonics in the keV X-ray Regime from Mid-Infrared Femtosecond Lasers," Science 336, 1287-1291 (2012). 
[9] R. J. England, R. J. Noble, K. Bane, D. H. Dowell, C.-K. Ng, J. E. Spencer, S. Tantawi, Z. Wu, R. L. Byer, E. Peralta, K. Soong, C.-M. Chang, B. Montazeri, S. J. Wolf, B. Cowan, J. Dawson, W. Gai, P. Hommelhoff, Y.-C. Huang, C. Jing, C. McGuinness, R. B. Palmer, B. Naranjo, J. Rosenzweig, G. Travish, A. Mizrahi, L. Schachter, C. Sears, G. R. Werner, and R. B. Yoder, "Dielectric laser accelerators," Reviews of Modern Physics 86, 1337-1389 (2014).

[10] D. J. Richardson, "Applied physics. Filling the light pipe." Science (New York, N.Y.) 330, 327-8 (2010).

[11] R.-J. Essiambre and R. W. Tkach, "Capacity Trends and Limits of Optical Communication Networks," Proceedings of the IEEE 100, 1035-1055 (2012).

[12] D. J. Richardson, J. M. Fini, and L. E. Nelson, "Space-division multiplexing in optical fibres," Nature Photonics 7, 354-362 (2013).

[13] G. P. Agrawal, Nonlinear Fiber Optics (Academic Press, 2007).

[14] S. S. L. Pitaevskii, Bose-Einstein Condensation (Oxford University Press, 2003).

[15] R. W. Boyd, Nonlinear Optics (Academic Press, 2008), 3rd ed.

[16] G. Genty, P. Kinsler, B. Kibler, and J. M. Dudley, "Nonlinear envelope equation modeling of sub-cycle dynamics and harmonic generation in nonlinear waveguides," Optics Express 15, 5382 (2007).

[17] M. Segev, Y. Silberberg, and D. N. Christodoulides, "Anderson localization of light," Nature Photonics 7, 197-204 (2013).

[18] L. Feng, R. El-Ganainy, and L. Ge, "Non-Hermitian photonics based on paritytime symmetry," Nature Photonics 11, 752-762 (2017).

[19] R. El-Ganainy, K. G. Makris, M. Khajavikhan, Z. H. Musslimani, S. Rotter, and D. N. Christodoulides, "Non-Hermitian physics and PT symmetry," Nature Physics 14, 11-19 (2018).

[20] J. A. Buck, Fundamentals of Optical Fibers (John Wiley \& Sons, 2004).

[21] A. Mafi, "Pulse Propagation in a Short Nonlinear Graded-Index Mul- 
timode Optical Fiber," Journal of Lightwave Technology 30, 2803-2811 (2012).

[22] F. Poletti and P. Horak, "Description of ultrashort pulse propagation in multimode optical fibers," Journal of the Optical Society of America B 25, 1645 (2008).

[23] P. Horak and F. Poletti, "Multimode Nonlinear Fiber Optics: Theory and Applications," in "Recent Progress in Optical Fiber Research," , M. Yasin, ed. (2012), chap. 1, pp. 3-24.

[24] R. W. Boyd, S. G. Lukishova, and Y. Shen, eds., Self-focusing: Past and Present: Fundamentals and Prospects (Springer, 2008).

[25] R.-J. Essiambre, G. J. Foschini, G. Kramer, and P. J. Winzer, “Capacity Limits of Information Transport in Fiber-Optic Networks," Physical Review Letters 101, 163901 (2008).

[26] A. Mecozzi, C. Antonelli, and M. Shtaif, "Modeling of Nonlinear Propagation in Space-Division Multiplexed Fiber-Optic Transmission," Journal of Lightwave Technology, Vol. 34, Issue 1, pp. 36-54 34, 36-54 (2016).

[27] L. G. Wright, W. H. Renninger, D. N. Christodoulides, and F. W. Wise, "Spatiotemporal dynamics of multimode optical solitons." Optics express 23, 3492-506 (2015).

[28] A. Hasegawa, "Self-confinement of multimode optical pulse in a glass fiber," Optics Letters 5, 416 (1980).

[29] B. Crosignani and P. Di Porto, "Soliton propagation in multimode optical fibers," Optics Letters 6, 329 (1981).

[30] S.-S. Yu, C.-H. Chien, Y. Lai, and J. Wang, "Spatio-temporal solitary pulses in graded-index materials with Kerr nonlinearity," Optics Communications 119, 167-170 (1995).

[31] S. Raghavan and G. P. Agrawal, "Spatiotemporal solitons in inhomogeneous nonlinear media," Optics Communications 180, 377-382 (2000).

[32] O. V. Shtyrina, M. P. Fedoruk, Y. S. Kivshar, and S. K. Turitsyn, "Coexistence of collapse and stable spatiotemporal solitons in multimode fibers," Physical Review A 97, 013841 (2018). 
[33] W. H. Renninger and F. W. Wise, "Optical solitons in graded-index multimode fibres." Nature communications 4, 1719 (2013).

[34] Y. Kivshar and G. Agrawal, Optical Solitons: From Fibers to Photonic Crystals [Hardcover] (Academic Press; 1 edition, 2003).

[35] Z. Zhu, L. G. Wright, D. N. Christodoulides, and F. W. Wise, "Observation of multimode solitons in few-mode fiber," Optics Letters 41, 4819 (2016).

[36] D. David and M. Tratnik, "Polarization modulated solitary waves in an optical fiber," Physica D: Nonlinear Phenomena 51, 308-315 (1991).

[37] D. Majus, G. Tamošauskas, I. Gražulevičit, N. Garejev, A. Lotti, A. Couairon, D. Faccio, and A. Dubietis, "Nature of Spatiotemporal Light Bullets in Bulk Kerr Media," Physical Review Letters 112, 193901 (2014).

[38] Y. Silberberg, “Collapse of optical pulses," Opt. Lett. 15, 1282-1284 (1990).

[39] W. H. Renninger, A. Chong, and F. W. Wise, "Pulse shaping and evolution in normal-dispersion mode-locked fiber lasers," IEEE Journal on Selected Topics in Quantum Electronics 18, 389-398 (2012).

[40] L. G. Wright, D. N. Christodoulides, and F. W. Wise, "Controllable spatiotemporal nonlinear effects in multimode fibres," Nature Photonics 9, 306-310 (2015).

[41] F. M. Mitschke and L. F. Mollenauer, "Discovery of the soliton selffrequency shift." Optics letters 11, 659-61 (1986).

[42] J. P. Gordon, "Theory of the soliton self-frequency shift." Optics letters 11, 662-4 (1986).

[43] J. H. Lee, J. Van Howe, C. Xu, and X. Liu, "Soliton self-frequency shift: Experimental demonstrations and applications," IEEE Journal on Selected Topics in Quantum Electronics 14, 713-723 (2008).

[44] K. Krupa, A. Tonello, A. Barthélémy, V. Couderc, B. M. Shalaby, A. Bendahmane, G. Millot, and S. Wabnitz, "Observation of Geometric Parametric Instability Induced by the Periodic Spatial Self-Imaging of Multimode Waves." Physical review letters 116, 183901 (2016). 
[45] L. G. Wright, S. Wabnitz, D. N. Christodoulides, and F. W. Wise, "Ultrabroadband Dispersive Radiation by Spatiotemporal Oscillation of Multimode Waves." Physical review letters 115, 223902 (2015).

[46] F. Matera, A. Mecozzi, M. Romagnoli, and M. Settembre, "Sideband instability induced by periodic power variation in long-distance fiber links," Optics Letters 18, 1499 (1993).

[47] A. Chong, J. Buckley, W. Renninger, and F. Wise, "All-normal-dispersion femtosecond fiber laser," Optics Express 14, 10095 (2006).

[48] K. Krupa, A. Tonello, B. M. Shalaby, M. Fabert, A. Barthélémy, G. Millot, S. Wabnitz, and V. Couderc, "Spatial beam self-cleaning in multimode fibres," Nature Photonics 11, 237-241 (2017).

[49] Z. Liu, L. G. Wright, D. N. Christodoulides, and F. W. Wise, "Kerr selfcleaning of femtosecond-pulsed beams in graded-index multimode fiber," Optics Letters 41, 3675 (2016).

[50] G. Lopez-Galmiche, Z. Sanjabi Eznaveh, M. A. Eftekhar, J. Antonio Lopez, L. G. Wright, F. Wise, D. Christodoulides, and R. Amezcua Correa, "Visible supercontinuum generation in a graded index multimode fiber pumped at 1064nm," Optics Letters 41, 2553 (2016).

[51] N. B. Terry, T. G. Alley, T. H. Russell, and K. T. Engel, “An explanation of SRS beam cleanup in graded-index fibers and the absence of SRS beam cleanup in step-index fibers," Optics Express 15, 17509 (2007).

[52] P. Aschieri, J. Garnier, C. Michel, V. Doya, and A. Picozzi, “Condensation and thermalization of classsical optical waves in a waveguide," Physical Review A 83, 033838 (2011).

[53] R. Guenard, K. Krupa, R. Dupiol, M. Fabert, A. Bendahmane, V. Kermene, A. Desfarges-Berthelemot, J. L. Auguste, A. Tonello, A. Barthélémy, G. Millot, S. Wabnitz, and V. Couderc, "Nonlinear beam self-cleaning in a coupled cavity composite laser based on multimode fiber," Optics Express 25, 22219 (2017).

[54] R. Guenard, K. Krupa, R. Dupiol, M. Fabert, A. Bendahmane, V. Kermene, A. Desfarges-Berthelemot, J. L. Auguste, A. Tonello, A. Barthélémy, G. Millot, S. Wabnitz, and V. Couderc, "Kerr self-cleaning of pulsed beam in an ytterbium doped multimode fiber," Optics Express 25, 4783 (2017). 
[55] K. Krupa, C. Louot, V. Couderc, M. Fabert, R. Guenard, B. M. Shalaby, A. Tonello, D. Pagnoux, P. Leproux, A. Bendahmane, R. Dupiol, G. Millot, and S. Wabnitz, "Spatiotemporal characterization of supercontinuum extending from the visible to the mid-infrared in a multimode graded-index optical fiber," Optics Letters 41, 5785 (2016).

[56] D. Ceoldo, K. Krupa, A. Tonello, V. Couderc, D. Modotto, U. Minoni, G. Millot, and S. Wabnitz, "Second harmonic generation in multimode graded-index fibers: spatial beam cleaning and multiple harmonic sideband generation," Optics Letters 42, 971 (2017).

[57] L. G. Wright, Z. Liu, D. A. Nolan, M.-J. Li, D. N. Christodoulides, and F. W. Wise, "Self-organized instability in graded-index multimode fibres," Nature Photonics 10, 771-776 (2016).

[58] P. Bak, C. Tang, and K. Wiesenfeld, "Self-organized criticality," Physical Review A 38, 364-374 (1988).

[59] D. L. Turcotte, "Self-organized criticality," Reports on Progress in Physics 62, 1377-1429 (1999).

[60] E. Nazemosadat, H. Pourbeyram, and A. Mafi, "Phase matching for spontaneous frequency conversion via four-wave mixing in graded-index multimode optical fibers," Journal of the Optical Society of America B 33, 144 (2016).

[61] D. Marcuse, Theory of Dielectric Optical Waveguides (Academic Press, 2012).

[62] K. P. Ho and J. M. Kahn, "Mode Coupling and its Impact on Spatially Multiplexed Systems," in "Optical Fiber Telecommunications VIB: Systems and Networks: Sixth Edition," (2013), pp. 491-568.

[63] S. Longhi and D. Janner, "Self-focusing and nonlinear periodic beams in parabolic index optical fibres," Journal of Optics B: Quantum and Semiclassical Optics 6, S303-S308 (2004).

[64] S. Longhi, "Modulational instability and space-time dynamics in nonlinear parabolic-index optical fibers." Optics letters 28, 2363-2365 (2003).

[65] L. W. Liou, X. D. Cao, C. J. McKinstrie, and G. P. Agrawal, "Spatiotemporal instabilities in dispersive nonlinear media," Physical Review A 46, 42024208 (1992). 
[66] K. O. Hill, D. C. Johnson, and B. S. Kawasaki, “Efficient conversion of light over a wide spectral range by four-photon mixing in a multimode gradedindex fiber." Applied optics 20, 1075-9 (1981).

[67] R. Dewar, "Information theory explanation of the fluctuation theorem, maximum entropy production and self-organized criticality in nonequilibrium stationary states," Journal of Physics A: Mathematical and General 36, 631-641 (2003).

[68] E. Ding, S. Lefrancois, J. N. Kutz, and F. W. Wise, "Scaling Fiber Lasers to Large Mode Area: An Investigation of Passive Mode-Locking Using a Multi-Mode Fiber." IEEE journal of quantum electronics 47, 597-606 (2011).

[69] I. P. Christov, H. C. Kapteyn, M. M. Murnane, C.-P. Huang, and J. Zhou, "Spacetime focusing of femtosecond pulses in a Ti:sapphire laser," Optics Letters 20, 309 (1995).

[70] I. P. Christov and V. D. Stoev, "Kerr-lens mode-locked laser model: role of space time effects," Journal of the Optical Society of America B 15, 1960 (1998).

[71] C. Jirauschek, F. X. Kärtner, and U. Morgner, "Spatiotemporal Gaussian pulse dynamics in Kerr-lens mode-locked lasers," Journal of the Optical Society of America B 20, 1356 (2003).

[72] A. Chong, L. G. Wright, and F. W. Wise, "Ultrafast fiber lasers based on self-similar pulse evolution: A review of current progress," (2015).

[73] L. G. Wright, D. N. Christodoulides, and F. W. Wise, "Spatiotemporal mode-locking in multimode fiber lasers," Science 358, 94-97 (2017).

[74] K. Regelskis, J. Želudevičius, K. Viskontas, and G. Račiukaitis, “Ytterbiumdoped fiber ultrashort pulse generator based on self-phase modulation and alternating spectral filtering," Optics Letters 40, 5255 (2015).

[75] Z. Liu, Z. M. Ziegler, L. G. Wright, and F. W. Wise, "Megawatt peak power from a Mamyshev oscillator," Optica 4, 649 (2017).

[76] P. Sidorenko, W. Fu, L. G. Wright, and F. W. Wise, "Multi-megawatt, selfseeded Mamyshev oscillator," (2018). 
[77] D. Auston, "Transverse mode locking," IEEE Journal of Quantum Electronics 4, 420-422 (1968).

[78] P. W. Smith, "Simultaneous phase-locking of longitudinal and transverse laser modes," Applied Physics Letters 13, 235-237 (1968).

[79] P. Smith, "Mode-locking of lasers," Proceedings of the IEEE 58, 1342-1357 (1970).

[80] K. Goda and B. Jalali, "Dispersive Fourier transformation for fast continuous single-shot measurements," (2013).

[81] J. Carpenter, B. J. Eggleton, and J. Schröder, "Observation of EisenbudWignerSmith states as principal modes in multimode fibr," European Conference on Optical Communication, ECOC 9, 751-757 (2014).

[82] G. Labroille, B. Denolle, P. Jian, P. Genevaux, N. Treps, and J.-F. Morizur, "Efficient and mode selective spatial mode multiplexer based on multiplane light conversion," Optics Express 22, 15599 (2014).

[83] O. Shapira, A. F. Abouraddy, J. D. Joannopoulos, and Y. Fink, "Complete Modal Decomposition for Optical Waveguides," Physical Review Letters 94, 143902 (2005).

[84] P. Sidorenko and O. Cohen, "Single-shot ptychography," Optica 3, 9 (2016).

[85] X. He, C. Liu, and J. Zhu, "Single-shot Fourier ptychography based on diffractive beam splitting," Optics Letters 43, 214 (2018).

[86] F. Stutzki, H.-J. Otto, F. Jansen, C. Gaida, C. Jauregui, J. Limpert, and A. Tünnermann, "High-speed modal decomposition of mode instabilities in high-power fiber lasers," Optics Letters 36, 4572 (2011).

[87] Z. Guang, M. Rhodes, and R. Trebino, "Measuring spatiotemporal ultrafast field structures of pulses from multimode optical fibers," Applied Optics 56, 3319 (2017).

[88] P. Bowlan, P. Gabolde, A. Shreenath, K. McGresham, R. Trebino, and S. Akturk, "Crossed-beam spectral interferometry: a simple, high-spectralresolution method for completely characterizing complex ultrashort pulses in real time," Optics Express 14, 11892 (2006). 
[89] C.-H. Lu, Y.-J. Tsou, H.-Y. Chen, B.-H. Chen, Y.-C. Cheng, S.-D. Yang, M.-C. Chen, C.-C. Hsu, and A. H. Kung, "Generation of intense supercontinuum in condensed media," Optica 1, 400 (2014).

[90] Y.-C. Cheng, C.-H. Lu, Y.-Y. Lin, and A. H. Kung, "Supercontinuum generation in a multi-plate medium," Optics Express 24, 7224 (2016).

[91] O. Tzang, A. M. Caravaca-Aguirre, and R. Piestun, "Wave-front shaping in nonlinear multimode fibers," (2017).

[92] R. Florentin, V. Kermene, J. Benoist, A. Desfarges-Berthelemot, D. Pagnoux, A. Barthélémy, and J.-P. Huignard, "Shaping the light amplified in a multimode fiber," Light: Science \& Applications 6, e16208 (2016). 